doi:10.1017/S1474748020000298 C The Author(s), 2020. Published by Cambridge University Press. This is an Open Access article, distributed under the terms of the Creative Commons Attribution licence (http://creativecommons.org/licenses/by/4.0), which permits unrestricted re-use, distribution, and reproduction in any medium, provided the original work is properly cited.

\title{
POINCARÉ AND SOBOLEV INEQUALITIES FOR DIFFERENTIAL FORMS IN HEISENBERG GROUPS AND CONTACT MANIFOLDS
}

\author{
ANNALISA BALDI ${ }^{\circledR 1}$, BRUNO FRANCHI ${ }^{\circledR 1}$ AND PIERRE PANSU ${ }^{2}$ \\ ${ }^{1}$ Università di Bologna, Dipartimento di Matematica, Piazza di Porta S. Donato \\ 5, 40126 Bologna, Italy (annalisa.baldi2@unibo.it; bruno.franchi@unibo.it) \\ ${ }^{2}$ Université Paris-Saclay, CNRS, Laboratoire de mathématiques d'Orsay, \\ 91405, Orsay, France (pierre.pansu@universite-paris-saclay.fr)
}

(Received 4 April 2019; revised 26 May 2020; accepted 26 May 2020;

first published online 29 June 2020)

\begin{abstract}
In this paper, we prove contact Poincaré and Sobolev inequalities in Heisenberg groups $\mathbb{H}^{n}$, where the word 'contact' is meant to stress that de Rham's exterior differential is replaced by the exterior differential of the so-called Rumin complex $\left(E_{0}^{\bullet}, d_{c}\right)$, which recovers the scale invariance under the group dilations associated with the stratification of the Lie algebra of $\mathbb{H}^{n}$. In addition, we construct smoothing operators for differential forms on sub-Riemannian contact manifolds with bounded geometry, which act trivially on cohomology. For instance, this allows us to replace a closed form, up to adding a controlled exact form, with a much more regular differential form.
\end{abstract}

Keywords: Heisenberg groups; differential forms; Sobolev-Poincaré inequalities; contact manifolds; homotopy formula

2010 Mathematics subject classification: Primary 58A10; 35R03; 53D10

Secondary 26D15; 43A80; 46E35

\section{Contents}

$\begin{array}{llr}\mathbf{1} & \text { Introduction } & \mathbf{8 7 0}\end{array}$

1.1 Sobolev and Poincaré inequalities for differential forms . . . . . . . . . . . 870

1.2 Contact manifolds . . . . . . . . . . . . . . . . . . . 871

1.3 Results on Poincaré and Sobolev inequalities . . . . . . . . . . . . . 873

1.4 State of the art . . . . . . . . . . . . . . . . . . . . . . . . . . . . . . . . . . . . . . . . .

1.5 Open questions . . . . . . . . . . . . . . . . . . . . . . . . . . . . . . . . . . . . . . . . . . . . . . . .

1.6 Global homotopy operators . . . . . . . . . . . . . . . . . . . . . . . . . . . . . . . . . . . . . . .

1.7 Local homotopy operators . . . . . . . . . . . . . . . . . . . . . . . . . . . . . . . . . . . . . . . . . . .

1.8 Global smoothing . . . . . . . . . . . . . . . . . . . . . . . . . . . . . . . . . . . . . . . . . . . . .

1.9 Structure of the paper . . . . . . . . . . . . . . . . 877

2 Heisenberg groups and the Rumin complex $\left(E_{0}^{\bullet}, d_{c}\right) \quad \mathbf{8 7 8}$

2.1 Differential forms on Heisenberg groups . . . . . . . . . . . . . 878 
2.2 The Rumin complex on Heisenberg groups . . . . . . . . . . . . . . . . 881

2.3 The Rumin complex in contact manifolds . . . . . . . . . . . . . . . 886

3 Kernels and Laplacians $\quad \mathbf{8 8 8}$

3.1 Kernels in Heisenberg groups . . . . . . . . . . . . . . . . . . . . . . 888

3.2 Rumin's Laplacians . . . . . . . . . . . . . . . . . . . . . . . 891

4 Function spaces $\quad \mathbf{8 9 4}$

4.1 Sobolev spaces on Heisenberg groups . . . . . . . . . . . . . . . . 894

4.2 Sobolev spaces on contact sub-Riemannian manifolds with bounded geometry ......................... 897

5 Homotopy formulas and Poincaré and Sobolev inequalities $\quad 899$

6 Contact manifolds and global smoothing $\quad 912$

$7 \quad$ Large scale geometry of contact sub-Riemannian manifolds $\quad 915$

7.1 Three-dimensional Lie groups . . . . . . . . . . . . . . . . . . . . . . 915

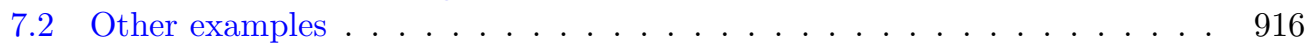

7.3 Further remarks . . . . . . . . . . . . . . . . 917

$\begin{array}{ll}\text { References } & 917\end{array}$

\section{Introduction}

\subsection{Sobolev and Poincaré inequalities for differential forms}

The Sobolev inequality in $\mathbb{R}^{n}$ states that if $u$ is a compactly supported function, then

$$
\|u\|_{q} \leqslant C_{p, q, n}\|d u\|_{p}
$$

whenever

$$
1 \leqslant p, q<+\infty, \quad \frac{1}{p}-\frac{1}{q}=\frac{1}{n},
$$

where $d u$ is the differential of $u$ (which is a 1 -form).

A local version, for functions supported in the unit ball, holds under the weaker assumption

$$
1 \leqslant p, q<+\infty, \quad \frac{1}{p}-\frac{1}{q} \leqslant \frac{1}{n} .
$$

The Poincaré inequality is a variant for functions $u$ defined, but not necessarily compactly supported, in the unit ball $B$. It states that there exists a real number $c_{u}$ such that

$$
\left\|u-c_{u}\right\|_{q} \leqslant C_{p, q, n}\|d u\|_{p} .
$$

Alternatively, for a given exact 1-form $\omega$ on $B$, there exists a function $u$ on $B$ such that $d u=\omega$ on $B$ and such that

$$
\|u\|_{q} \leqslant C_{p, q, n}\|\omega\|_{p}
$$


This suggests the following generalization for higher-degree differential forms in Riemannian manifolds.

Let $M$ be a Riemannian manifold with or without boundary. We say that a global Poincaré inequality holds on $M$ if there exists a positive constant $C=C(M, p, q)$ such that for every exact $h$-form $\omega$ on $M$, belonging to $L^{p}$, there exists an $(h-1)$-form $\phi$ such that $d \phi=\omega$ and

$$
\|\phi\|_{q} \leqslant C\|\omega\|_{p} .
$$

Shortly, we shall say that Poincaré $p, q(h)$ holds.

A global Sobolev inequality holds on $M$ if for every exact compactly supported $h$-form $\omega$ on $M$, belonging to $L^{p}$, there exists a compactly supported $(h-1)$-form $\phi$ such that $d \phi=\omega$ and

$$
\|\phi\|_{q} \leqslant C\|\omega\|_{p} .
$$

Again, we shall say that $\operatorname{Sobolev}_{p, q}(h)$ holds.

In both statements, the assumption that given forms are exact is there to separate the topological problem (whether a given closed form is exact) from the analytical one (whether a primitive can be upgraded to one that satisfies estimates).

For bounded convex domains, the global Poincaré and Sobolev inequalities hold for $1<$ $p<\infty$ (see, respectively, [33, Corollary 4.2] and [42, Theorem 4.1 and equation (169)]). However, for more general Euclidean domains, the validity of the Poincaré inequality is sensitive to irregularities of boundaries. One way to eliminate such a dependence is to allow a loss on domain. For the case $p=1$ in the Euclidean setting, we refer the reader to [4].

Say an interior Poincaré inequality Poincaré $p, q(h)$ holds on $M$ if for every small enough $r>0$ and large enough $\lambda \geqslant 1$, there exists a constant $C=C(M, p, q, r, \lambda)$ such that for every $x \in M$ and every exact $h$-form $\omega$ on $B(x, \lambda r)$, belonging to $L^{p}$, there exists an $(h-1)$-form $\phi$ on $B(x, r)$ such that $d \phi=\omega$ on $B(x, r)$ and

$$
\|\phi\|_{L^{q}(B(x, r))} \leqslant C\|\omega\|_{L^{p}(B(x, \lambda r))} .
$$

By interior Sobolev inequalities, we mean that, if $\omega$ is supported in $B(x, r)$, then there exists $\phi$ supported in $B(x, \lambda r)$ such that $d \phi=\omega$ and

$$
\|\phi\|_{L^{q}(B(x, \lambda r))} \leqslant C\|\omega\|_{L^{p}(B(x, r))} .
$$

It turns out that in several situations, the loss on domain is harmless. This is the case for $L^{q, p}$-cohomological applications; see [47].

Let us comment on the terminology. Due to the loss on domain, inequality (1) provides no information on the behavior of differential forms near the boundary of their domain of definition. This is why we speak of an interior Poincaré inequality.

\subsection{Contact manifolds}

A contact structure on an odd-dimensional manifold $M$ is a smooth distribution of hyperplanes $H$, which is maximally non-integrable in the following sense: if $\theta$ is a locally 
defined smooth 1 -form such that $H=\operatorname{ker}(\theta)$, then $d \theta$ restricts to a non-degenerate 2-form on $H$, i.e., if $2 n+1$ is the dimension of $M$, then $\theta \wedge(d \theta)^{n} \neq 0$ on $M$ (see [41, Proposition 3.41]). A contact manifold $(M, H)$ is the data of a smooth manifold $M$ and a contact structure $H$ on $M$. Contact diffeomorphisms (also called contactomorphisms; see Definition 2.13) are contact structure preserving diffeomorphisms between contact manifolds. The prototype of a contact manifold is the Heisenberg group $\mathbb{H}^{n}$, the simply connected Lie group whose Lie algebra is the central extension

$$
\mathfrak{h}=\mathfrak{h}_{1} \oplus \mathfrak{h}_{2}, \quad \text { with } \mathfrak{h}_{2}=\mathbb{R}=Z(\mathfrak{h}),
$$

with bracket $\mathfrak{h}_{1} \otimes \mathfrak{h}_{1} \rightarrow \mathfrak{h}_{2}=\mathbb{R}$ being a non-degenerate skew-symmetric 2 -form. The contact structure is obtained by left-translating $\mathfrak{h}_{1}$. According to a theorem by Darboux, every contact manifold is locally contactomorphic to $\mathbb{H}^{n}$. The Heisenberg Lie algebra admits a one-parameter group of automorphisms $\delta_{t}$,

$$
\delta_{t}=t \text { on } \mathfrak{h}_{1}, \quad \delta_{t}=t^{2} \text { on } \mathfrak{h}_{2},
$$

which are counterparts of the usual Euclidean dilations in $\mathbb{R}^{n}$. Thus, differential forms on $\mathfrak{h}$ split into two eigenspaces under $\delta_{t}$. Therefore, the de Rham complex lacks scale invariance under these anisotropic dilations.

A substitute for the de Rham complex, which recovers scale invariance under $\delta_{t}$, has been defined by Rumin [50]. It makes sense for arbitrary contact manifolds $(M, H)$ and it is invariant under contactomorphisms.

Let $h=0, \ldots, 2 n+1$. Rumin's substitute for smooth differential forms of degree $h$ is the smooth sections of a vector bundle $E_{0}^{h}$. If $h \leqslant n, E_{0}^{h}$ is a sub-bundle of $\Lambda^{h} H^{*}$. If $h \geqslant n, E_{0}^{h}$ is a sub-bundle of $\Lambda^{h} H^{*} \otimes(T M / H)$. Rumin's substitute for de Rham's exterior differential is a linear differential operator $d_{c}$ from sections of $E_{0}^{h}$ to sections of $E_{0}^{h+1}$ such that $d_{c}^{2}=0$.

We stress that the operator $d_{c}$ has order 2 when $h=n$ and order 1 otherwise.

This phenomenon will be a major issue in the proofs of our results and will affect the choice of the exponents $p, q$ in our inequalities.

The data of $(M, H)$ equipped with a scalar product $g$, defined on sub-bundle $H$ only, is called a sub-Riemannian contact manifold and we shall write $(M, H, g)$. The scalar product on $H$ determines a choice of a local contact form $\theta$ and hence a norm on the line bundle $T M / H$. Therefore $E_{0}^{h}$ are endowed with a scalar product. Using $\theta \wedge(d \theta)^{n}$ as a volume form, one gets $L^{p}$-norms on spaces of smooth Rumin differential forms.

In any sub-Riemannian contact manifold $(M, H, g)$, we can define a sub-Riemannian distance $d_{M}$ (see e.g., [43]) inducing on $M$ the same topology of $M$ as a manifold. In particular, Heisenberg groups $\mathbb{H}^{n}$ can be viewed as sub-Riemannian contact manifolds. If we choose on the contact sub-bundle of $\mathbb{H}^{n}$ a left-invariant metric, it turns out that the associated sub-Riemannian metric is also left-invariant. It is customary to call this distance in $\mathbb{H}^{n}$ a Carnot-Carathéodory distance.

Poincaré and Sobolev inequalities for differential forms make sense on contact sub-Riemannian manifolds: merely replace the exterior differential $d$ with $d_{c}$. All left-invariant sub-Riemannian metrics on the Heisenberg group are bi-Lipschitz 
equivalent, and hence we may refer to the sub-Riemannian Heisenberg group without referring to a specific left-invariant metric: if a Poincaré inequality holds for some left-invariant metric, it holds for all of them. On the other hand, in the absence of symmetry assumptions, large scale behaviors of sub-Riemannian contact manifolds are diverse. Examples illustrating this phenomenon will be given in $\S 7$.

\subsection{Results on Poincaré and Sobolev inequalities}

In this paper, we prove global $\mathbb{H}$-Poincaré and $\mathbb{H}$-Sobolev inequalities and interior $\mathbb{H}$-Poincaré and $\mathbb{H}$-Sobolev inequalities in Heisenberg groups, where the prefix $\mathbb{H}$ is meant to stress that the exterior differential is replaced with Rumin's exterior differential $d_{c}$. The range of parameters differs slightly from the Euclidean case due to the fact that $d_{c}$ has order 2 in middle dimension. Let $h \in\{0, \ldots, 2 n+1\}$. We say that assumption $E(h, p, q, n)$ holds if $1<p \leqslant q<\infty$ satisfy

$$
\frac{1}{p}-\frac{1}{q}= \begin{cases}\frac{1}{2 n+2} & \text { if } h \neq n+1 \\ \frac{2}{2 n+2} & \text { if } h=n+1 .\end{cases}
$$

Say that assumption $I(h, p, q, n)$ holds if $1<p \leqslant q<\infty$ satisfy

$$
\frac{1}{p}-\frac{1}{q} \leqslant \begin{cases}\frac{1}{2 n+2} & \text { if } h \neq n+1 \\ \frac{2}{2 n+2} & \text { if } h=n+1\end{cases}
$$

Theorem 1.1. Under assumption $E(h, p, q, n)$, global $\mathbb{H}$-Poincaré $_{p, q}$ and $\mathbb{H}$-Sobolev $p, q$ inequalities hold for Rumin's h-forms on $\mathbb{H}^{n}$.

Theorem 1.2. Under assumption $I(h, p, q, n)$, interior $\mathbb{H}$-Poincaré $p, q$ and interior $\mathbb{H}$-Sobolev $_{p, q}$ inequalities hold for Rumin's $h$-forms on $\mathbb{H}^{n}$.

Precise formulations of interior Poincaré and Sobolev inequalities are given in $\S 5$.

Remark 1.3. We stress that the core of the present paper is the proof of the interior inequalities of Theorem 1.2. In fact, since $p>1$, the global estimates of Theorem 1.1 are more or less straightforward consequences of the $L^{p}-L^{q}$ continuity of singular integrals of potential type (see $\S 1.6)$.

Here is a simple consequence of these results. Combining both theorems with results from [47], we get the following corollary.

Corollary 1.4. Under assumption $E(h, p, q, n)$, the $\ell^{q, p}$-cohomology in degree $h$ of $\mathbb{H}^{n}$ vanishes.

Our third result is the construction of a smoothing homotopy on general contact manifolds. Under a bounded geometry assumption, uniform estimates can be given 
(precise definitions of bounded geometry contact manifolds, as well as of associated Sobolev spaces $W^{j, p}$, will be given in $\left.\S 4.2\right)$.

Theorem 1.5. Let $k \geqslant 3$ be an integer. Let $(M, H, g)$ be a $2 n+1$-dimensional subRiemannian contact manifold of bounded $C^{k}$-geometry. Under assumption $I(h, p, q, n)$, there exist operators $S_{M}$ and $T_{M}$ on h-forms on $M$, which are bounded from $W^{j, p}$ to $W^{j, q}$ for all $0 \leqslant j \leqslant k-1$, and such that

$$
1=S_{M}+d_{c} T_{M}+T_{M} d_{c} .
$$

Furthermore, $S_{M}$ and $T_{M}$ are bounded from $W^{j-1, p}$ to $W^{j, p}$ if $j \geqslant 1$ (resp. from $W^{j-2, p}$ to $W^{j, p}$ if $j \geqslant 2$ and degree $h=n+1$ ).

We stress that the 'approximate homotopy formula' (4) has no consequences for the cohomology of $M$. The iteration of the process yields an operator $S_{M}$, which is bounded from $L^{p}$ to $W^{k-1, q}$, and still acts trivially on cohomology. For instance, it is possible to replace a closed form with a much more regular differential form (up to adding a controlled exact form).

\subsection{State of the art}

This paper is part of a larger project aimed to prove $(p, q)$-Poincaré and Sobolev inequalities in Heisenberg groups when $1 \leqslant p<q \leqslant \infty$. Thus it seems convenient to point out the different cases we have to deal with. Let us restrict ourselves for a while to Euclidean spaces $\mathbb{R}^{n}$ and Heisenberg groups $\mathbb{H}^{n}$. The first fundamental distinction is the following:

(i) global inequalities (i.e., inequalities on all the space $\mathbb{R}^{n}$ or $\mathbb{H}^{n}$ );

(ii) interior inequalities (for instance on Carnot-Carathéodory balls).

For each one of the above geometric assumptions, we must distinguish between

(iii) the case $p=1$ and

(iv) the case $p>1$.

In the scalar case, $(p, q)$-Poincaré and Sobolev inequalities are well understood both in Euclidean spaces and in Heisenberg groups for all $p \geqslant 1$. Consider now differential forms of higher degree.

For the case $p=1$, global inequalities in $\mathbb{R}^{n}$ (Gagliardo-Nirenberg inequalities for differential forms) have been proved by Bourgain \& Brezis [15] and Lanzani \& Stein [36] via a suitable identity for closed differential forms and relying on careful estimates for divergence-free vector fields. Thanks to the counterpart of this identity proved by Chanillo $\&$ van Schaftingen in homogeneous groups [18], similar global inequalities for differential forms in $\mathbb{H}^{n}$ were proved in [3]. We stress that in [3], algebra plays an important role precisely in the proof of the identities for closed forms. Therefore apart from Heisenberg groups, only a handful of more general nilpotent groups have been treated [11].

Interior inequalities when $p=1$ use the estimate of [3] combined with an approximate homotopy formula introduced in the present paper, but require a new different argument to control the commutator between Rumin's exterior differential (or de Rham's exterior 
differential in $\mathbb{R}^{n}$ ) and multiplication by a cut-off function. These inequalities are proved for Heisenberg groups in [6] and in [4] for Euclidean spaces. Note that in the Heisenberg group case, one more algebraic obstacle shows up, averages of $L^{1}$ forms; see [49].

Consider now the case $p>1$. In the Euclidean setting, interior Poincaré inequalities for $p>1$ are proved in [33]. However, the arguments of [33] do not extend to Heisenberg groups. Thus, the core of the present paper is the proof of interior Poincare and Sobolev inequalities in $\mathbb{H}^{n}$ when $p>1$. Indeed, as we shall point out later (see Remark 1.3), when $p>1$, global inequalities in $\mathbb{H}^{n}$ (as well as in $\mathbb{R}^{n}$ ) are more or less straightforward.

On the contrary, interior inequalities require a different more sophisticated argument (see $\S 1.7$ for a gist of our proof). At the same time, the techniques introduced in the present paper differ substantially from those of [3] for global inequalities for $p=1$.

The case when $q=\infty$ can be obtained by duality, and this will appear in [5]. We refer the reader also to [7] for endpoint inequalities in Orlicz spaces.

For more general sub-Riemannian spaces, the strategy is to reduce to large scale invariants (see $\S 7$ ). For this, one must pass via interior inequalities and a global smoothing procedure, like in Theorems 1.2 and 1.5. In particular, in the present paper and in [6] we deal with a special class of sub-Riemannian manifolds, the sub-Riemannian contact manifold of bounded $C^{k}$-geometry as in Definition 4.9 .

\subsection{Open questions}

Keeping in mind the analogous inequalities in the scalar case, the following (still open) questions naturally arise.

(1) Do Poincaré and Sobolev inequalities hold without loss of the domain for some family of specific domains as, e.g., for metric balls associated with a left-invariant homogeneous distance?

(2) Since Heisenberg groups provide the simplest non-commutative instance of arbitrary Carnot groups (connected, simply connected stratified nilpotent groups; see [45]), the following question naturally arises: How much of these results do extend to more general Carnot groups?

Let us make a few comments about the previous questions.

(1) When dealing with scalar functions it is possible to obtain $\mathbb{H}$-Poincaré ${ }_{p, q}$ inequalities on Carnot-Carathéodory balls without loss on the domain and the argument relies on the so-called Boman chain condition (see, e.g., [22, 24]). However, it is not clear at all how to extend this technique to differential forms.

(2) The argument used in this paper relies on an appropriate approximate homotopy formula (see point $(2)$ in $\S 1.7$ ). It is reasonable to expect that the construction of the approximate homotopy operator could be generalized to more general Carnot groups using the construction carried out in $[8,48]$ to prove a compensated compactness result (see formulas (37) and (38) in [8]). However, for Carnot groups, we expect only unsharp estimates due to the crucial role of a fundamental solution of a 0-order Laplace operator mixing up components of forms of different homogeneity. 
Further comments related to this question can be found in Remark 5.22, where specific examples in more general Carnot groups are given.

Let us give now a sketch of the proofs.

\subsection{Global homotopy operators}

The most efficient way to prove a Poincaré inequality is to find a homotopy between identity and 0 on the complex of differential forms, i.e., a linear operator $K$ that raises the degree by 1 and satisfies

$$
I=d K+K d
$$

More generally, we shall deal with homotopies between identity and other operators $P$, i.e., of the form

$$
I-P=d K+K d .
$$

In Euclidean space, the Laplacian provides us with such a homotopy. Write $\Delta=$ $d \delta+\delta d$. Denote by $\Delta^{-1}$ the operator of convolution with the fundamental solution of the Laplacian. Then $\Delta^{-1}$ commutes with $d$ and its adjoint $\delta$; hence $K_{\text {Euc }}=\delta \Delta^{-1}$ satisfies $I=d K_{\text {Euc }}+K_{\text {Euc }} d$ on globally defined $L^{p}$ differential forms. Furthermore, $K_{\text {Euc }}$ is bounded $L^{p} \rightarrow L^{q}$ provided $\frac{1}{p}-\frac{1}{q}=\frac{1}{n}$. This proves the global Poincaré $p, q(h)$ inequality for Euclidean space.

Rumin defines a Laplacian $\Delta_{c}$ by $\Delta_{c}=d_{c} \delta_{c}+\delta_{c} d_{c}$ when both $d_{c}$ and $\delta_{c}$ are first-order horizontal differential operators, and by $\Delta_{c}=\left(d_{c} \delta_{c}\right)^{2}+\delta_{c} d_{c}$ or $\Delta_{c}=d_{c} \delta_{c}+\left(\delta_{c} d_{c}\right)^{2}$ near middle dimension (i.e., when $h=n$ or $h=n+1$, respectively), when one of them has order 2. This leads to a homotopy of the form $K_{0}=\delta_{c} \Delta_{c}^{-1}$ or $K_{0}=\delta_{c} d_{c} \delta_{c} \Delta_{c}^{-1}$ depending on the degree. Again, $K_{0}$ is a singular integral of potential type associated with a homogeneous kernel and therefore is bounded from $L^{p}$ to $L^{q}$ under assumption $E(h, p, q, n)$ (see [20] or [21] for the continuity of Riesz potentials in homogeneous groups). This proves the global $\mathbb{H}$-Poincaré $p, q(h)$ inequality for Heisenberg group, Theorem 1.1.

\subsection{Local homotopy operators}

We pass to interior estimates. In Euclidean space, Poincaré's lemma asserts that every closed form on a ball is exact. We need a quantitative version of this statement. The standard proof of Poincaré's lemma relies on a homotopy operator, which depends on the choice of an origin. Averaging over origins yields a bounded operator $K_{\text {Euc }}: L^{p} \rightarrow L^{q}$, as was observed by Iwaniec and Lutoborski [33]. This proves the global Euclidean Poincaré $_{p, q}(h)$ inequality for convex Euclidean domains. A support preserving variant $J_{\text {Euc }}: L^{p} \rightarrow L^{q}$ appears in Mitrea et al. [42], and this proves the global Euclidean Sobolev $_{p, q}$ inequality for bounded convex Euclidean domains. Incidentally, since for balls, constants do not depend on the radius of the ball, this reproves the global Euclidean Sobolev $_{p, q}$ inequality for Euclidean spaces.

In this paper, a sub-Riemannian counterpart is obtained using the homotopy equivalence of the de Rham and Rumin complexes. Since this homotopy is a differential operator, a preliminary smoothing operation is needed. This is obtained by localizing 
(multiplying the kernel with cut-offs) the global homotopy $K_{0}$ provided by the inverse of Rumin's (modified) Laplacian.

Hence the proof goes as follows (see $\S 5$ ):

(1) Show that the inverse $K_{0}$ of Rumin's modified Laplacian on all of $\mathbb{H}^{n}$ is given by a homogeneous kernel $k_{0}$. Deduce bounds $L^{p} \rightarrow W^{1, q}$, where $q, p$ are as above. Conclude that $K_{0}$ is an exact homotopy for globally defined $L^{p}$ forms. Basically, this step does not contain any new idea, relying only on the estimates of the fundamental solution of Rumin's modified Laplacian (see [10]) and on classical estimates for convolution kernels in homogeneous groups (see [20,21]).

(2) Take a smooth cut-off function $\psi, \psi \equiv 1$ in a neighborhood of the origin, and split $k_{0}=\psi k_{0}+(1-\psi) k_{0}$, so that $\psi k_{0}$ has small support near the origin and $(1-\psi) k_{0}$ is smooth. Denote by $T$ the convolution operator associated with the kernel $\psi k_{0}$, and by $K_{\text {smooth }}$ the convolution operator associated with the kernel $(1-\psi) k_{0}$. It turns out that $T$ is a homotopy on balls (with a loss on domain) between the identity $I$ and the operator $S:=d_{c} K_{\text {smooth }}+K_{\text {smooth }} d_{c}$ (which is smoothing), i.e., $I-S=d_{c} T+T d_{c}$. The operator $S$ provides the required local smoothing operator.

(3) Compose Iwaniec \& Lutoborski's averaged Poincaré homotopy for the de Rham complex and Rumin's homotopy, and apply the result to smoothed forms. This proves an interior Poincaré inequality in Heisenberg groups. Replacing Iwaniec \& Lutoborski's homotopy with Mitrea, Mitrea \& Monniaux's homotopy leads to an interior Sobolev inequality in Heisenberg groups.

\subsection{Global smoothing}

Now we piece together local homotopy operators into globally defined smoothing operators. Let $k \geqslant 3$. Let $(M, H, g)$ be a bounded $C^{k}$-geometry sub-Riemannian contact manifold. Pick a uniform covering by equal radius balls. Let $\chi_{j}$ be a partition of unity subordinate to this covering. Let $\phi_{j}$ be the corresponding charts from the unit Heisenberg ball. Let $S_{j}$ and $T_{j}$ denote the smoothing and homotopy operators associated with $\phi_{j}$ using the pull-back operator. Set

$$
T=\sum_{j} T_{j} \chi_{j}, \quad S=\sum_{j} S_{j} \chi_{j}+T_{j}\left[\chi_{j}, d_{c}\right] .
$$

When $d_{c}$ is first order, the commutator $\left[\chi_{j}, d_{c}\right]$ is an order 0 differential operator; hence $T_{j}\left[\chi_{j}, d_{c}\right]$ gains one derivative. When $d_{c}$ is second order, $\left[\chi_{j}, d_{c}\right]$ is a first-order differential operator. It turns out that precisely in this case, $T_{j}$ gains two derivatives. Hence $T_{j}\left[\chi_{j}, d_{c}\right]$ gains one derivative in this case as well.

The details are discussed in $\S 6$.

\subsection{Structure of the paper}

In $\S 2$, we collect basic results about Heisenberg groups $\mathbb{H}^{n}$ and differential forms in $\mathbb{H}^{n}$. Successively, we recall the notion of the Rumin complex for Heisenberg groups as well as for general contact manifolds, providing explicit examples in low dimensions. In $\S 3$, we present a list of general results for Folland-Stein homogeneous kernels and, in 
particular, for matrix-valued kernels associated with Rumin's homogeneous Laplacian in $\mathbb{H}^{n}$. Section 4 is devoted to the theory of Folland-Stein Sobolev spaces in Heisenberg groups and in sub-Riemannian contact manifolds with bounded geometry. In particular, in $\S 4.2$, we precisely provide the notion and the properties of manifolds with bounded geometry. Section 5 is the core of the paper, containing an approximate homotopy formula (i.e., a homotopy formula with a smoothing error term) and Poincaré and Sobolev inequalities for differential forms in $\mathbb{H}^{n}$. Then, in $\S 6$, we are able to prove a similar approximate homotopy formula for sub-Riemannian contact manifolds with bounded geometry. The error term is a regularizing operator with 'maximal regularity'. Finally, $\S 7$ contains a few examples of contact manifolds with bounded geometry and a brief discussion of the $\ell^{q, p}$ cohomology.

\section{Heisenberg groups and the Rumin complex $\left(E_{0}^{\bullet}, d_{c}\right)$}

\subsection{Differential forms on Heisenberg groups}

We denote by $\mathbb{H}^{n}$ the $(2 n+1)$-dimensional Heisenberg group, identified with $\mathbb{R}^{2 n+1}$ through exponential coordinates. A point $p \in \mathbb{H}^{n}$ is denoted by $p=(x, y, t)$, with both $x, y \in \mathbb{R}^{n}$ and $t \in \mathbb{R}$. If $p$ and $p^{\prime} \in \mathbb{H}^{n}$, the group operation is defined by

$$
p \cdot p^{\prime}=\left(x+x^{\prime}, y+y^{\prime}, t+t^{\prime}+\frac{1}{2} \sum_{j=1}^{n}\left(x_{j} y_{j}^{\prime}-y_{j} x_{j}^{\prime}\right)\right) .
$$

Note that $\mathbb{H}^{n}$ can be equivalently identified with $\mathbb{C} \times \mathbb{R}$ endowed with the group operation

$$
(z, t) \cdot(\zeta, \tau):=\left(z+\zeta, t+\tau-\frac{1}{2} \operatorname{Im}(z \bar{\zeta})\right)
$$

The unit element of $\mathbb{H}^{n}$ is the origin, which will be denoted by $e$. For any $q \in \mathbb{H}^{n}$, the (left) translation $\tau_{q}: \mathbb{H}^{n} \rightarrow \mathbb{H}^{n}$ is defined as

$$
p \mapsto \tau_{q} p:=q \cdot p .
$$

For a general review on Heisenberg groups and their properties, we refer the reader to $[30,56]$ and to [57]. We limit ourselves to fixing some notations, following [27].

First, we note that Heisenberg groups are smooth manifolds (and therefore are Lie groups). In particular, the pull-back of differential forms is well defined as follows (see, e.g., [28, Proposition 1.106]).

Definition 2.1. If $\mathcal{U}, \mathcal{V}$ are open subsets of $\mathbb{H}^{n}$, and $f: \mathcal{U} \rightarrow \mathcal{V}$ is a diffeomorphism, then for any differential form $\alpha$ of degree $h$, we denote by $f^{\sharp} \alpha$ the pull-back form in $U$ defined by

$$
\left(f^{\sharp} \alpha\right)(p)\left(v_{1}, \ldots, v_{h}\right):=\alpha(f(p))\left(d f(p) v_{1}, \ldots, d f(p) v_{h}\right)
$$

for any $h$-tuple $\left(v_{1}, \ldots, v_{h}\right)$ of tangent vectors at $p$.

The Heisenberg group $\mathbb{H}^{n}$ can be endowed with the homogeneous norm (Cygan-Korányi norm): if $p=(x, y, t) \in \mathbb{H}^{n}$, then we set

$$
\varrho(p)=\left(\left(x^{2}+y^{2}\right)^{2}+16 t^{2}\right)^{1 / 4},
$$


and we define the gauge distance (a true distance, see [56, p. 638], with a different normalization in the group law, which is left-invariant, i.e., $d\left(\tau_{q} p, \tau_{q} p^{\prime}\right)=d\left(p, p^{\prime}\right)$ for all $\left.p, p^{\prime} \in \mathbb{H}^{n}\right)$ as

$$
d(p, q):=\varrho\left(p^{-1} \cdot q\right) .
$$

Note that $d$ is equivalent to the Carnot-Carathéodory distance on $\mathbb{H}^{n}$ (see, e.g., [14, Corollary 5.1.5]). Finally, the balls for the metric $d$ are the so-called Cygan-Korányi balls

$$
B(p, r):=\left\{q \in \mathbb{H}^{n} ; d(p, q)<r\right\} .
$$

Note that Cygan-Korányi balls are convex smooth sets.

A straightforward computation shows that, if $\rho(p)<1$, then

$$
|p| \leqslant \rho(p) \leqslant|p|^{1 / 2} \text {. }
$$

It is well known that the topological dimension of $\mathbb{H}^{n}$ is $2 n+1$, since as a smooth manifold it coincides with $\mathbb{R}^{2 n+1}$, whereas the Hausdorff dimension of $\left(\mathbb{H}^{n}, d\right)$ is $Q:=$ $2 n+2$ (the so-called homogeneous dimension of $\mathbb{H}^{n}$ ).

We denote by $\mathfrak{h}$ the Lie algebra of the left-invariant vector fields of $\mathbb{H}^{n}$. The standard basis of $\mathfrak{h}$ is given, for $i=1, \ldots, n$, by

$$
X_{i}:=\partial_{x_{i}}-\frac{1}{2} y_{i} \partial_{t}, \quad Y_{i}:=\partial_{y_{i}}+\frac{1}{2} x_{i} \partial_{t}, \quad T:=\partial_{t} .
$$

The only non-trivial commutation relations are $\left[X_{j}, Y_{j}\right]=T$, for $j=1, \ldots, n$. The horizontal subspace $\mathfrak{h}_{1}$ is the subspace of $\mathfrak{h}$ spanned by $X_{1}, \ldots, X_{n}$ and $Y_{1}, \ldots, Y_{n}$ : $\mathfrak{h}_{1}:=\operatorname{span}\left\{X_{1}, \ldots, X_{n}, Y_{1}, \ldots, Y_{n}\right\}$.

Coherently, from now on, we refer to $X_{1}, \ldots, X_{n}, Y_{1}, \ldots, Y_{n}$ (identified with first-order differential operators) as the horizontal derivatives. Denoting by $\mathfrak{h}_{2}$ the linear span of $T$, the two-step stratification of $\mathfrak{h}$ is expressed by

$$
\mathfrak{h}=\mathfrak{h}_{1} \oplus \mathfrak{h}_{2} .
$$

The stratification of the Lie algebra $\mathfrak{h}$ induces a family of non-isotropic dilations $\delta_{\lambda}$ : $\mathbb{H}^{n} \rightarrow \mathbb{H}^{n}, \lambda>0$ as follows: if $p=(x, y, t) \in \mathbb{H}^{n}$, then

$$
\delta_{\lambda}(x, y, t)=\left(\lambda x, \lambda y, \lambda^{2} t\right)
$$

The vector space $\mathfrak{h}$ can be endowed with an inner product, indicated by $\langle\cdot, \cdot\rangle$, making $X_{1}, \ldots, X_{n}, Y_{1}, \ldots, Y_{n}$ and $T$ orthonormal.

Throughout this paper, we write also

$$
W_{i}:=X_{i}, \quad W_{i+n}:=Y_{i} \quad \text { and } \quad W_{2 n+1}:=T, \quad \text { for } i=1, \ldots, n .
$$

The dual space of $\mathfrak{h}$ is denoted by $\bigwedge^{1} \mathfrak{h}$. The basis of $\bigwedge^{1} \mathfrak{h}$, dual to the basis $\left\{X_{1}, \ldots\right.$, $\left.Y_{n}, T\right\}$, is the family of covectors $\left\{d x_{1}, \ldots, d x_{n}, d y_{1}, \ldots, d y_{n}, \theta\right\}$, where

$$
\theta:=d t-\frac{1}{2} \sum_{j=1}^{n}\left(x_{j} d y_{j}-y_{j} d x_{j}\right)
$$


is called the contact form in $\mathbb{H}^{n}$. We denote by $\langle\cdot, \cdot\rangle$ the inner product in $\bigwedge^{1} \mathfrak{h}$ that makes $\left(d x_{1}, \ldots, d y_{n}, \theta\right)$ an orthonormal basis.

Coherently with the previous notation (10), we set

$$
\omega_{i}:=d x_{i}, \quad \omega_{i+n}:=d y_{i} \quad \text { and } \quad \omega_{2 n+1}:=\theta, \quad \text { for } i=1, \ldots, n .
$$

We put $\bigwedge_{0} \mathfrak{h}:=\bigwedge^{0} \mathfrak{h}=\mathbb{R}$ and, for $1 \leqslant h \leqslant 2 n+1$,

$$
\bigwedge^{h} \mathfrak{h}:=\operatorname{span}\left\{\omega_{i_{1}} \wedge \cdots \wedge \omega_{i_{h}}: 1 \leqslant i_{1}<\cdots<i_{h} \leqslant 2 n+1\right\} .
$$

In the sequel, we shall denote by $\Theta^{h}$ the basis of $\bigwedge^{h} \mathfrak{h}$ defined by

$$
\Theta^{h}:=\left\{\omega_{i_{1}} \wedge \cdots \wedge \omega_{i_{h}}: 1 \leqslant i_{1}<\cdots<i_{h} \leqslant 2 n+1\right\} .
$$

To avoid cumbersome notations, if $I:=\left(i_{1}, \ldots, i_{h}\right)$, we write

$$
\omega_{I}:=\omega_{i_{1}} \wedge \cdots \wedge \omega_{i_{h}} .
$$

The inner product $\langle\cdot, \cdot \cdot\rangle$ on $\bigwedge^{1} \mathfrak{h}$ yields naturally an inner product $\langle\cdot, \cdot\rangle$ on $\bigwedge^{h} \mathfrak{h}$ making $\Theta^{h}$ an orthonormal basis.

The volume $(2 n+1)$-form $\omega_{1} \wedge \cdots \wedge \omega_{2 n+1}$ will be also written as $d V$.

Throughout this paper, the elements of $\wedge^{h} \mathfrak{h}$ are identified with left-invariant differential forms of degree $h$ on $\mathbb{H}^{n}$.

Definition 2.2. A $h$-form $\alpha$ on $\mathbb{H}^{n}$ is said to be left-invariant if

$$
\tau_{q}^{\#} \alpha=\alpha \quad \text { for any } q \in \mathbb{H}^{n} .
$$

The same construction can be performed starting from the vector subspace $\mathfrak{h}_{1} \subset \mathfrak{h}$, obtaining the horizontal $h$-covectors

$$
\bigwedge^{h} \mathfrak{h}_{1}:=\operatorname{span}\left\{\omega_{i_{1}} \wedge \cdots \wedge \omega_{i_{h}}: 1 \leqslant i_{1}<\cdots<i_{h} \leqslant 2 n\right\} .
$$

It is easy to see that

$$
\Theta_{0}^{h}:=\Theta^{h} \cap \bigwedge^{h} \mathfrak{h}_{1}
$$

provides an orthonormal basis of $\bigwedge^{h} \mathfrak{h}_{1}$.

Keeping in mind that the Lie algebra $\mathfrak{h}$ can be identified with the tangent space to $\mathbb{H}^{n}$ at $x=e$ (see, e.g., [28, Proposition 1.72]), starting from $\bigwedge^{h} \mathfrak{h}$ we can define by left translation a fiber bundle over $\mathbb{H}^{n}$ that we can still denote by $\bigwedge^{h} \mathfrak{h}$. We can think of $h$-forms as sections of $\bigwedge^{h} \mathfrak{h}$. We denote by $\Omega^{h}$ the vector space of all smooth $h$-forms.

We already pointed out in $\S 1.2$ that the stratification of the Lie algebra $\mathfrak{h}$ yields a lack of homogeneity of de Rham's exterior differential with respect to group dilations $\delta_{\lambda}$. Thus, to take into account the different degrees of homogeneity of the covectors when they vanish on different layers of the stratification, we introduce the notion of weight of a covector as follows.

Definition 2.3. If $\eta \neq 0, \eta \in \bigwedge^{1} \mathfrak{h}_{1}$, we say that $\eta$ has weight 1 , and we write $w(\eta)=1$. If $\eta=\theta$, we say $w(\eta)=2$. More generally, if $\eta \in \bigwedge^{h} \mathfrak{h}, \eta \neq 0$, we say that $\eta$ has pure weight $p$ if $\eta$ is a linear combination of covectors $\omega_{i_{1}} \wedge \cdots \wedge \omega_{i_{h}}$ with $w\left(\omega_{i_{1}}\right)+\cdots+w\left(\omega_{i_{h}}\right)=p$. 
Note that if $\eta, \zeta \in \bigwedge^{h} \mathfrak{h}$ and $w(\eta) \neq w(\zeta)$, then $\langle\eta, \zeta\rangle=0$ (see [8, Remark 2.4]). We note also that $w(d \theta)=w(\theta)$.

We stress that generic covectors may fail to have a pure weight: it is enough to consider $\mathbb{H}^{1}$ and the covector $d x_{1}+\theta \in \bigwedge^{1} \mathfrak{h}$. However, the following result holds (see $[8$, formula $(16)])$ :

$$
\bigwedge^{h} \mathfrak{h}=\bigwedge^{h, h} \mathfrak{h} \oplus \bigwedge^{h, h+1} \mathfrak{h}=\bigwedge^{h} \mathfrak{h}_{1} \oplus\left(\bigwedge^{h-1} \mathfrak{h}_{1}\right) \wedge \theta
$$

where $\bigwedge^{h, p} \mathfrak{h}$ denotes the linear span of the $h$-covectors of weight $p$. By our previous remark, decomposition (12) is orthogonal. In addition, since the elements of the basis $\Theta^{h}$ have pure weights, a basis of $\bigwedge^{h, p} \mathfrak{h}$ is given by $\Theta^{h, p}:=\Theta^{h} \cap \bigwedge^{h, p} \mathfrak{h}$ (such a basis is usually called an adapted basis).

We note that, according to (12), the weight of an $h$-form is either $h$ or $h+1$ and there are no forms of weight $h+2$ since there is only one 1 -form of weight 2 . Something analogous can be possible for instance in $\mathbb{H}^{n} \times \mathbb{R}$, but it fails to be possible already in the case of general step 2 groups with higher-dimensional center (see also Remark 5.22).

As above, starting from $\bigwedge^{h, p} \mathfrak{h}$, we can define by left translation a fiber bundle over $\mathbb{H}^{n}$ that we can still denote by $\bigwedge^{h, p} \mathfrak{h}$. Thus, if we denote by $\Omega^{h, p}$ the vector space of all smooth $h$-forms in $\mathbb{H}^{n}$ of weight $p$, i.e., the space of all smooth sections of $\bigwedge^{h, p} \mathfrak{h}$, we have

$$
\Omega^{h}=\Omega^{h, h} \oplus \Omega^{h, h+1} \text {. }
$$

\subsection{The Rumin complex on Heisenberg groups}

Let us give a short introduction to the Rumin complex. For a more detailed presentation, we refer the reader to Rumin's papers [53]. Here we follow the presentation of [8]. The exterior differential $d$ does not preserve weights. It splits into

$$
d=d_{0}+d_{1}+d_{2},
$$

where $d_{0}$ preserves weight, $d_{1}$ increases weight by 1 unit and $d_{2}$ increases weight by 2 units.

More explicitly, let $\alpha \in \Omega^{h}$ be a (say) smooth form of pure weight $h$. We can write

$$
\alpha=\sum_{\omega_{I} \in \Theta_{0}^{h}} \alpha_{I} \omega_{I}, \quad \text { with } \alpha_{I} \in \mathcal{C}^{\infty}\left(\mathbb{H}^{n}\right)
$$

Then

$$
d \alpha=\sum_{\omega_{I} \in \Theta_{0}^{h}} \sum_{j=1}^{2 n}\left(W_{j} \alpha_{I}\right) \omega_{j} \wedge \omega_{I}+\sum_{\omega_{I} \in \Theta_{0}^{h}}\left(T \alpha_{I}\right) \theta \wedge \omega_{I}=d_{1} \alpha+d_{2} \alpha
$$

and $d_{0} \alpha=0$. On the other hand, if $\alpha \in \Omega^{h, h+1}$ has pure weight $h+1$, then

$$
\begin{gathered}
\alpha=\sum_{\omega_{J} \in \Theta_{0}^{h-1}} \alpha_{J} \theta \wedge \omega_{J}, \\
d \alpha=\sum_{\omega_{J} \in \Theta_{0}^{h}} \alpha_{J} d \theta \wedge \omega_{J}+\sum_{\omega_{J} \in \Theta_{0}^{h}} \sum_{j=1}^{2 n}\left(W_{j} \alpha_{J}\right) \omega_{j} \wedge \theta \wedge \omega_{I}=d_{0} \alpha+d_{1} \alpha,
\end{gathered}
$$

and $d_{2} \alpha=0$. 
It is crucial to note that $d_{0}$ is an algebraic operator in the sense that for any real-valued $f \in \mathcal{C}^{\infty}\left(\mathbb{H}^{n}\right)$, we have

$$
d_{0}(f \alpha)=f d_{0} \alpha
$$

so that its action can be identified at any point with the action of a linear operator from $\bigwedge^{h} \mathfrak{h}$ to $\bigwedge^{h+1} \mathfrak{h}$ (which we denote again by $\left.d_{0}\right)$.

Following Rumin [51, 53], we give the next definition.

Definition 2.4. If $0 \leqslant h \leqslant 2 n+1$, keeping in mind that $\bigwedge^{h} \mathfrak{h}$ is endowed with a canonical inner product, we set

$$
E_{0}^{h}:=\operatorname{ker} d_{0} \cap\left(\operatorname{Im} d_{0}\right)^{\perp} .
$$

Straightforwardly, $E_{0}^{h}$ inherits from $\bigwedge^{h} \mathfrak{h}$ the inner product.

As above, $E_{0}^{\bullet}$ defines by left translation a fiber bundle over $\mathbb{H}^{n}$, which we still denote by $E_{0}^{\bullet}$. To avoid cumbersome notations, we denote also by $E_{0}^{\bullet}$ the space of sections of this fiber bundle.

Let $L: \bigwedge^{h} \mathfrak{h} \rightarrow \bigwedge^{h+2} \mathfrak{h}$ be the Lefschetz operator defined by

$$
L \xi=d \theta \wedge \xi
$$

Then the spaces $E_{0}^{\bullet}$ can be defined explicitly as follows.

Theorem 2.5 (See [50, 52]). We have the following:

(i) $E_{0}^{1}=\bigwedge^{1} \mathfrak{h}_{1}$;

(ii) if $2 \leqslant h \leqslant n$, then $E_{0}^{h}=\bigwedge^{h} \mathfrak{h}_{1} \cap\left(\bigwedge^{h-2} \mathfrak{h}_{1} \wedge d \theta\right)^{\perp}$ (i.e., $E_{0}^{h}$ is the space of the so-called primitive covectors of $\left.\bigwedge^{h} \mathfrak{h}_{1}\right)$;

(iii) if $n<h \leqslant 2 n+1$, then $E_{0}^{h}=\left\{\alpha=\beta \wedge \theta, \beta \in \wedge^{h-1} \mathfrak{h}_{1}, \gamma \wedge d \theta=0\right\}=\theta \wedge \operatorname{ker} L$;

(iv) if $1<h \leqslant n$, then $N_{h}:=\operatorname{dim} E_{0}^{h}=\left(\begin{array}{c}2 n \\ h\end{array}\right)-\left(\begin{array}{c}2 n \\ h-2\end{array}\right)$;

(v) if $*$ denotes the Hodge duality associated with the inner product in $\wedge^{\bullet} \mathfrak{h}$ and the volume form $d V$, then $* E_{0}^{h}=E_{0}^{2 n+1-h}$.

Note that all forms in $E_{0}^{h}$ have weight $h$ if $1 \leqslant h \leqslant n$ and weight $h+1$ if $n<h \leqslant 2 n+1$.

A further geometric interpretation (in terms of decomposition of $\mathfrak{h}$ and of graphs within $\left.\mathbb{H}^{n}\right)$ can be found in [26].

Note that there exists a left-invariant orthonormal basis

$$
\Xi_{0}^{h}=\left\{\xi_{1}^{h}, \ldots, \xi_{\operatorname{dim} E_{0}^{h}}^{h}\right\}
$$

of $E_{0}^{h}$ that is adapted to filtration (12). Such a basis is explicitly constructed by induction in [1].

The core of Rumin's theory consists in the construction of a suitable 'exterior differential' $d_{c}: E_{0}^{h} \rightarrow E_{0}^{h+1}$, making $\mathcal{E}_{0}:=\left(E_{0}^{\bullet}, d_{c}\right)$ a complex homotopic to the de Rham complex.

Let us sketch Rumin's construction: first, the next result (see [8, Lemma 2.11] for a proof) allows us to define a (pseudo) inverse of $d_{0}$ : 
Lemma 2.6. If $1 \leqslant h \leqslant n$, then $\operatorname{ker} d_{0}=\bigwedge^{h} \mathfrak{h}_{1}$. Moreover, if $\beta \in \bigwedge^{h+1} \mathfrak{h}$, then there exists a unique $\gamma \in \bigwedge^{h} \mathfrak{h} \cap\left(\operatorname{ker} d_{0}\right)^{\perp}$ such that

$$
d_{0} \gamma-\beta \in \mathcal{R}\left(d_{0}\right)^{\perp} .
$$

With the notations of the previous lemma, we set

$$
\gamma:=d_{0}^{-1} \beta
$$

We note that $d_{0}^{-1}$ preserves the weights.

The following theorem summarizes the construction of the intrinsic differential $d_{c}$ (for details, see [53] and [8, Section 2]).

Theorem 2.7. The de Rham complex $\left(\Omega^{\bullet}, d\right)$ splits into the direct sum of two sub-complexes $\left(E^{\bullet}, d\right)$ and $\left(F^{\bullet}, d\right)$, with

$$
E:=\operatorname{ker} d_{0}^{-1} \cap \operatorname{ker}\left(d_{0}^{-1} d\right) \quad \text { and } \quad F:=\mathcal{R}\left(d_{0}^{-1}\right)+\mathcal{R}\left(d d_{0}^{-1}\right) .
$$

Let $\Pi_{E}$ be the projection on $E$ along $F$ (which is not an orthogonal projection). We have the following:

(i) If $\gamma \in E_{0}^{h}$, then

- $\Pi_{E} \gamma=\gamma-d_{0}^{-1} d_{1} \gamma$ if $1 \leqslant h \leqslant n$;

- $\Pi_{E} \gamma=\gamma$ if $h>n$.

(ii) $\Pi_{E}$ is a chain map, i.e.,

$$
d \Pi_{E}=\Pi_{E} d .
$$

(iii) Let $\Pi_{E_{0}}$ be the orthogonal projection from $\wedge^{*} \mathfrak{h}$ on $E_{0}^{\bullet}$; then

$$
\Pi_{E_{0}}=I-d_{0}^{-1} d_{0}-d_{0} d_{0}^{-1}, \quad \Pi_{E_{0}^{\perp}}=d_{0}^{-1} d_{0}+d_{0} d_{0}^{-1} .
$$

(iv) $\Pi_{E_{0}} \Pi_{E} \Pi_{E_{0}}=\Pi_{E_{0}}$ and $\Pi_{E} \Pi_{E_{0}} \Pi_{E}=\Pi_{E}$.

Set now

$$
d_{c}=\Pi_{E_{0}} d \Pi_{E}: E_{0}^{h} \rightarrow E_{0}^{h+1}, \quad h=0, \ldots, 2 n .
$$

We have the following:

(v) $d_{c}^{2}=0$.

(vi) The complex $E_{0}:=\left(E_{0}^{\bullet}, d_{c}\right)$ is homotopic to the de Rham complex.

(vii) $d_{c}: E_{0}^{h} \rightarrow E_{0}^{h+1}$ is a homogeneous differential operator in the horizontal derivatives of order 1 if $h \neq n$, whereas $d_{c}: E_{0}^{n} \rightarrow E_{0}^{n+1}$ is a homogeneous differential operator in the horizontal derivatives of order 2.

To illustrate the previous construction, let us write explicitly the classes $E_{0}^{h}$ and the differential $d_{c}: E_{0}^{h} \rightarrow E_{0}^{h+1}$ in $\mathbb{H}^{1}$ and $\mathbb{H}^{2}$ (for proofs, see e.g., [2]). 
Example 2.8. Consider the first Heisenberg group $\mathbb{H}^{1} \equiv \mathbb{R}^{3}$ with variables $(x, y, t)$. With the notations of (11), we have

$$
\begin{aligned}
& E_{0}^{1}=\operatorname{span}\{d x, d y\} \\
& E_{0}^{2}=\operatorname{span}\{d x \wedge \theta, d y \wedge \theta\} \\
& E_{0}^{3}=\operatorname{span}\{d x \wedge d y \wedge \theta\} .
\end{aligned}
$$

Thus, if $\alpha=\alpha_{1} d x+\alpha_{2} d y \in E_{0}^{1}$, then

(a) $d_{c} \alpha=\left(X^{2} \alpha_{2}-2 X Y \alpha_{1}+Y X \alpha_{1}\right) d x \wedge \theta+\left(2 Y X \alpha_{2}-Y^{2} \alpha_{1}-X Y \alpha_{2}\right) d y \wedge \theta$,

(b) $d_{c}^{*} \alpha=-\left(X \alpha_{1}+Y \alpha_{2}\right)$.

On the other hand, if $\alpha=\alpha_{13} d x \wedge \theta+\alpha_{23} d y \wedge \theta \in E_{0}^{2}$, then

(c) $d_{c} \alpha=\left(X \alpha_{23}-Y \alpha_{13}\right) d x \wedge d y \wedge \theta$,

(d) $d_{c}^{*} \alpha=\left(X Y \alpha_{13}-2 Y X \alpha_{13}-Y^{2} \alpha_{23}\right) d x+\left(X^{2} \alpha_{13}+2 X Y \alpha_{23}-Y X \alpha_{23}\right) d y$.

Example 2.9. Choose now $\mathbb{H}^{2} \equiv \mathbb{R}^{5}$, with variables $\left(x_{1}, x_{2}, y_{1}, y_{2}, t\right)$.

In this case,

$$
\begin{aligned}
E_{0}^{1}=\operatorname{span}\{ & \left\{d x_{1}, d x_{2}, d y_{1}, d y_{2}\right\} \\
E_{0}^{2}=\operatorname{span}\{ & \left\{d x_{1} \wedge d x_{2}, d x_{1} \wedge d y_{2}, d x_{2} \wedge d y_{1}, d y_{1} \wedge d y_{2},\right. \\
& \left.\frac{1}{\sqrt{2}}\left(d x_{1} \wedge d y_{1}-d x_{2} \wedge d y_{2}\right)\right\} .
\end{aligned}
$$

The classes $E_{0}^{3}$ and $E_{0}^{4}$ are easily written by Hodge duality:

$$
\begin{aligned}
& E_{0}^{3}=\operatorname{span}\{\left\{y_{1} \wedge d y_{2} \wedge \theta, d x_{2} \wedge d y_{1} \wedge \theta, d x_{1} \wedge d y_{2} \wedge \theta, d x_{1} \wedge d x_{2} \wedge \theta,\right. \\
&\left.\frac{1}{\sqrt{2}}\left(d x_{1} \wedge d y_{1}-d x_{2} \wedge d y_{2}\right) \wedge \theta\right\} \\
& E_{0}^{4}=\operatorname{span}\left\{d x_{2} \wedge d y_{1} \wedge d y_{2} \wedge \theta, d x_{1} \wedge d y_{1} \wedge d y_{2} \wedge \theta, d x_{1} \wedge d x_{2} \wedge d y_{2} \wedge \theta,\right. \\
&\left.d x_{1} \wedge d x_{2} \wedge d y_{1} \wedge \theta\right\} \\
& E_{0}^{5}= \operatorname{span}\left\{d x_{1} \wedge d x_{2} \wedge d y_{1} \wedge d y_{2} \wedge \theta=d V\right\} .
\end{aligned}
$$

Thus, if $\alpha=\alpha_{1} d x_{1}+\alpha_{2} d x_{2}+\alpha_{3} d y_{1}+\alpha_{4} d y_{2} \in E_{0}^{1}$, we have

$$
\text { (a) } \begin{aligned}
d_{c} \alpha= & \left(X_{1} \alpha_{2}-X_{2} \alpha_{1}\right) d x_{1} \wedge d x_{2}+\left(Y_{1} \alpha_{4}-Y_{2} \alpha_{3}\right) d y_{1} \wedge d y_{2} \\
& +\left(X_{1} \alpha_{4}-Y_{2} \alpha_{1}\right) d x_{1} \wedge d y_{2}+\left(X_{2} \alpha_{3}-Y_{1} \alpha_{2}\right) d x_{2} \wedge d y_{1} \\
& +\frac{X_{1} \alpha_{3}-Y_{1} \alpha_{1}-X_{2} \alpha_{4}+Y_{2} \alpha_{2}}{\sqrt{2}} \frac{1}{\sqrt{2}}\left(d x_{1} \wedge d y_{1}-d x_{2} \wedge d y_{2}\right)
\end{aligned}
$$

(b) $\delta_{c} \alpha=-\left(X_{1} \alpha_{1}+X_{2} \alpha_{2}+Y_{1} \alpha_{3}+Y_{2} \alpha_{4}\right)$. 
Finally, if

$$
\begin{aligned}
\alpha= & \alpha_{1} d x_{1} \wedge d x_{2}+\alpha_{2} d x_{1} \wedge d y_{2}+\alpha_{3} d x_{2} \wedge d y_{1}+\alpha_{4} d y_{1} \wedge d y_{2} \\
& +\frac{\alpha_{5}}{\sqrt{2}}\left(d x_{1} \wedge d y_{1}-d x_{2} \wedge d y_{2}\right) \in E_{0}^{2}
\end{aligned}
$$

we have

$$
\begin{aligned}
\text { (c) } d_{c} \alpha= & \left(Y_{1}^{2} \alpha_{2}-Y_{2}^{2} \alpha_{3}+\left(X_{1} Y_{1}-2 Y_{1} X_{1}-Y_{2} X_{2}\right) \alpha_{4}-\sqrt{2} Y_{1} Y_{2} \alpha_{5}\right) d y_{1} \wedge d y_{2} \wedge \theta \\
& +\left(-Y_{1}^{2} \alpha_{1}+\left(X_{1} Y_{1}-2 Y_{1} X_{1}+X_{2} Y_{2}\right) \alpha_{3}+X_{2}^{2} \alpha_{4}+\sqrt{2} X_{2} Y_{1} \alpha_{5}\right) d x_{2} \wedge d y_{1} \wedge \theta \\
& +\left(Y_{2}^{2} \alpha_{1}+\left(2 X_{1} Y_{1}-Y_{1} X_{1}-Y_{2} X_{2}\right) \alpha_{2}-X_{1}^{2} \alpha_{4}-\sqrt{2} X_{1} Y_{2} \alpha_{5}\right) d x_{1} \wedge d y_{2} \wedge \theta \\
& +\left(\left(2 X_{1} Y_{1}-Y_{1} X_{1}-2 X_{2} Y_{2}\right) \alpha_{1}+X_{2}^{2} \alpha_{2}+X_{1}^{2} \alpha_{3}-\sqrt{2} X_{1} X_{2} \alpha_{5}\right) d x_{1} \wedge d x_{2} \wedge \theta \\
& +\left(2 \sqrt{2} Y_{1} Y_{2} \alpha_{1}-2 \sqrt{2} X_{2} Y_{1} \alpha_{2}+2 \sqrt{2} X_{1} Y_{2} \alpha_{3}+2 \sqrt{2} X_{1} X_{2} \alpha_{4}+3 T \alpha_{5}\right) \\
& \cdot \frac{1}{\sqrt{2}}\left(d x_{1} \wedge d y_{1}-d x_{2} \wedge d y_{2}\right) \wedge \theta . \\
\text { (d) } \delta_{c} \alpha= & \left(X_{2} \alpha_{1}+Y_{2} \alpha_{2}+\frac{1}{\sqrt{2}} Y_{1} \alpha_{5}\right) d x_{1}+\left(-X_{1} \alpha_{1}+Y_{1} \alpha_{3}-\frac{1}{\sqrt{2}} Y_{2} \alpha_{5}\right) d x_{2} \\
& +\left(-X_{2} \alpha_{3}+Y_{2} \alpha_{4}-\frac{1}{\sqrt{2}} X_{1} \alpha_{5}\right) d y_{1}+\left(-X_{1} \alpha_{2}-Y_{1} \alpha_{4}+\frac{1}{\sqrt{2}} X_{2} \alpha_{5}\right) d y_{2} .
\end{aligned}
$$

Remark 2.10. The construction of the Rumin complex can be carried out in general Carnot groups following verbatim the construction presented in $\S 2.2$ for Heisenberg groups, once a general notion of weight is provided. This can be easily done in terms of homogeneity of a covector with respect to group dilations (see $[8,53,54])$.

Since the exterior differential $d_{c}$ on $E_{0}^{h}$ can be written in coordinates as a left-invariant homogeneous differential operator in the horizontal variables, of order 1 if $h \neq n$ and of order 2 if $h=n$, the proof of the following Leibniz formula is easy.

Lemma 2.11. If $\zeta$ is a smooth real function, then we have the following:

- If $h \neq n$, then on $E_{0}^{h}$ we have

$$
\left[d_{c}, \zeta\right]=P_{0}^{h},
$$

where $P_{0}^{h}: E_{0}^{h} \rightarrow E_{0}^{h+1}$ is a linear homogeneous differential operator of degree zero, with coefficients depending only on the horizontal derivatives of $\zeta$.

- If $h=n$, then on $E_{0}^{n}$ we have

$$
\left[d_{c}, \zeta\right]=P_{1}^{n}+P_{0}^{n}
$$

where $P_{1}^{n}: E_{0}^{n} \rightarrow E_{0}^{n+1}$ is a linear homogeneous differential operator of degree 1, with coefficients depending only on the horizontal derivatives of $\zeta$, and where $P_{0}^{h}: E_{0}^{n} \rightarrow$ $E_{0}^{n+1}$ is a linear homogeneous differential operator in the horizontal derivatives of degree 0 with coefficients depending only on second-order horizontal derivatives of $\zeta$. 
The next remarkable property of the Rumin complex is its invariance under contact transformations. Here we state a special case before developing this point in $\S 2.3$ (see [9, Proposition 3.19] for a proof).

Proposition 2.12. If we write a form $\alpha=\sum_{j} \alpha_{j} \xi_{j}^{h}$ in coordinates with respect to a left-invariant basis of $E_{0}^{h}($ see (15)), we have

$$
\tau_{q}^{\#} \alpha=\sum_{j}\left(\alpha_{j} \circ \tau_{q}\right) \xi_{j}^{h}
$$

for all $q \in \mathbb{H}^{n}$. In addition, for $t>0$,

$$
\delta_{t}^{\#} \alpha=t^{h} \sum_{j}\left(\alpha_{j} \circ \delta_{t}\right) \xi_{j}^{h} \quad \text { if } 1 \leqslant h \leqslant n
$$

and

$$
\delta_{t}^{\#} \alpha=t^{h+1} \sum_{j}\left(\alpha_{j} \circ \delta_{t}\right) \xi_{j}^{h} \quad \text { if } n+1 \leqslant h \leqslant 2 n+1 .
$$

\subsection{The Rumin complex in contact manifolds}

Let us start with the following definition (see [41, Section I-3]).

Definition 2.13. If $\left(M_{1}, H_{1}\right)$ and $\left(M_{2}, H_{2}\right)$ are contact manifolds with $H_{i}=\operatorname{ker} \alpha_{i}$ (i.e., $\alpha_{i}$ are contact forms), $i=1,2, \mathcal{U}_{1} \subset M_{1}, \mathcal{U}_{2} \subset M_{2}$ are open sets and $f$ is a diffeomorphism from $\mathcal{U}_{1}$ onto $\mathcal{U}_{2}$, then $f$ is said to be a contact diffeomorphisms if there exists a non-vanishing real function $\tau$ defined in $\mathcal{U}_{1}$ such that

$$
f^{\#} \alpha_{2}=\tau \alpha_{1} \quad \text { in } \mathcal{U}_{1}
$$

We recall that, by a classical theorem of Darboux, any contact manifold $(M, H)$ is locally contact diffeomorphic to the Heisenberg group $\mathbb{H}^{n}$ (see [41, p. 112]).

Rumin's intrinsic complex is invariantly defined for general contact manifolds $(M, H)$. Although the operators $d_{0}$ and $d_{0}^{-1}$ are not invariantly defined, the subspaces $E$ and $F$ of differential forms, the operator $\Pi_{E}$ onto $E$ parallel to $F$, the vector bundles $E_{0}^{h}$ and the projector $\Pi_{E_{0}}$ are contact invariants. To see this, let us follow [54].

Locally, $H$ is the kernel of a smooth contact 1-form $\theta$. Let $L: \wedge^{\bullet} H^{*} \rightarrow \wedge^{\bullet} H^{*}$ denote multiplication by $d \theta_{\mid H}$ (recall (14)).

Let us start with $E$ and $F$. It is well known that, for every $h \leqslant n-1, L^{n-h}: \bigwedge^{h} H^{*} \rightarrow$ $\bigwedge^{2 n-h} H^{*}$ is an isomorphism. It follows that $\operatorname{ker}\left(L^{n-h+1}\right)$ is a complement of $\mathcal{R}(L)$ in $\bigwedge^{h} H^{*}$, if $h \leqslant n$, and that $\mathcal{R}(L)=\bigwedge^{h} H^{*}$ if $h \geqslant n+1$. Therefore we set

$$
V^{h}= \begin{cases}\left\{\alpha \in T^{*} M ; L^{n-h+1}\left(\alpha_{\mid H}\right)=0\right\} & \text { if } h \leqslant n \\ \left\{\alpha \in T^{*} M ; \alpha_{\mid H}=0\right\} & \text { otherwise }\end{cases}
$$


Similarly, $\mathcal{R}\left(L^{h-n+1}\right)$ is a complement of $\operatorname{ker}(L)$ in $\bigwedge^{h} H^{*}$ if $h \geqslant n$, and $\operatorname{ker}(L)=\{0\}$ in $\wedge^{h} H^{*}$ if $h \leqslant n-1$. Therefore we set

$$
W^{h}= \begin{cases}\left\{\alpha \in T^{*} M ; \alpha_{\mid H}=0\right\} & \text { if } h \leqslant n-1, \\ \left\{\alpha \in T^{*} M ; \alpha \in \theta \wedge \mathcal{R}\left(L^{h-n+1}\right)\right\} & \text { otherwise }\end{cases}
$$

Changing $\theta$ to another smooth 1-form $\theta^{\prime}=f \theta$ with kernel $H$ does not change $V$ and $W$. With these choices, spaces of smooth sections of $V$ and $W$ (which we still denote by $V$ and $W$ ) depend only on the plane field $H$. We can define subspaces of smooth differential forms $E=V \cap d^{-1} V$ and $F=W+d W$ and the projector $\Pi_{E}$. Since no extra choices are involved, $E, F$ and $\Pi_{E}$ are invariant under contactomorphisms. On the Heisenberg group, one recovers the spaces $E$ and $F$ defined in Theorem 2.7.

Next, we define the sub-bundles $E_{0}^{h}$. In degrees $h \geqslant n+1, E_{0}^{h}=\theta \wedge\left(\bigwedge^{h} H^{*} \cap \operatorname{ker}(L)\right)$ is a contact invariant. Since

$$
\left(\Pi_{E_{0}}\right)_{\mid E}=\left(\left(\Pi_{E}\right)_{\mid E_{0}}\right)^{-1},
$$

the operator $d_{c}=\left(\left(\Pi_{E}\right)_{\mid E_{0}}\right)^{-1} \circ d \circ\left(\Pi_{E}\right)_{\mid E_{0}}$ is a contact invariant.

In degrees $h \leqslant n$, the restriction of differential forms to $H$ is an isomorphism of $E_{0}^{h}$ to $E_{0}^{\prime h}:=\bigwedge^{h} H^{*} \cap \operatorname{ker}\left(L^{n-h+1}\right)$. We note that for a differential form $\omega$ such that $\omega_{\mid H} \in E_{0}^{\prime}$, $\Pi_{E}(\omega)$ only depends on $\omega_{\mid H}$. It follows that $\left(\Pi_{E}\right)_{\mid E_{0}}$ can be viewed as defined on the space of sections of $E_{0}^{\prime}$ (still denoted by $E_{0}^{\prime}$ ), which is a contact invariant. Since

$$
\left(\Pi_{E_{0}}\right)_{\mid E}=\left(\left(\Pi_{E}\right)_{\mid E_{0}}\right)^{-1}, \quad \text { it follows that }\left(\Pi_{E_{0}^{\prime}}\right)_{\mid E}=\left(\left(\Pi_{E}\right)_{\mid E_{0}^{\prime}}\right)^{-1}
$$

and $d_{c}$ viewed as an operator on $E_{0}^{\prime}$,

$$
\left(\left(\Pi_{E}\right)_{\mid E_{0}^{\prime}}\right)^{-1} \circ d \circ\left(\Pi_{E}\right)_{\mid E_{0}^{\prime}}
$$

is a contact invariant. In the sequel, we shall ignore the distinction between $E_{0}$ and $E_{0}^{\prime}$. We shall denote $E_{0}^{\bullet}=\bigoplus_{h} E_{0}^{h}$ endowed with the exterior differential $d_{c}$.

Alternative contact invariant descriptions of the Rumin complex can be found in $[13$, $16]$.

By construction, we have the following:

(i) $d_{c}^{2}=0$;

(ii) The complex $\mathcal{E}_{0}:=\left(E_{0}^{\bullet}, d_{c}\right)$ is homotopically equivalent to the de Rham complex $\Omega:=\left(\Omega^{\bullet}, d\right)$. Thus, if $D \subset \mathbb{H}^{n}$ is an open set, unambiguously we write $H^{h}(D)$ for the $h$ th cohomology group.

(iii) $d_{c}: E_{0}^{h} \rightarrow E_{0}^{h+1}$ is a homogeneous differential operator in the horizontal derivatives of order 1 if $h \neq n$, whereas $d_{c}: E_{0}^{n} \rightarrow E_{0}^{n+1}$ is a homogeneous differential operator in the horizontal derivatives of order 2 .

The following statement expresses the fact that the Rumin complex is invariant under contactomorphism. In other words, the pull-back map is natural, i.e., it is a chain map for $\left(E_{0}^{\bullet}, d_{c}\right)$. 
Proposition 2.14. If $\phi$ is a contactomorphism from an open set $\mathcal{U} \subset \mathbb{H}^{n}$ to $M$, and we denote by $\mathcal{V}$ the open set $\mathcal{V}:=\phi(\mathcal{U})$, the pull-back operator $\phi^{\#}$ satisfies the following:

(i) $\phi^{\#} E_{0}^{\bullet}(\mathcal{V})=E_{0}^{\bullet}(\mathcal{U})$.

(ii) $d_{c} \phi^{\#}=\phi^{\#} d_{c}$.

(iii) If $\zeta$ is a smooth function in $M$, then the differential operator in $\mathcal{U} \subset \mathbb{H}^{n}$ defined by $v \rightarrow \phi^{\#}\left[d_{c}, \zeta\right]\left(\phi^{-1}\right)^{\#} v$ is a differential operator of order 0 if $v \in E_{0}^{h}(\mathcal{U}), h \neq n$ and a differential operator of order 1 if $v \in E_{0}^{n}(\mathcal{U})$.

Proof. Assertions (i) and (ii) follow straightforwardly since $\phi$ is a contact map. Assertion (iii) follows from Lemma 2.11 since, by definition,

$$
\phi^{\#}\left[d_{c}, \zeta\right]\left(\phi^{-1}\right)^{\#} v=\left[d_{c}, \zeta \circ \phi\right] v .
$$

\section{Kernels and Laplacians}

\subsection{Kernels in Heisenberg groups}

Following a classical notation [55], if $U \subset \mathbb{H}^{n}$ is an open set, we denote by $\mathcal{D}(U)$ the space of smooth functions in $U$ with compact support, by $\mathcal{D}^{\prime}(U)$ the space of distributions in $U$, and by $\mathcal{E}^{\prime}(U)$ the space of compactly supported distributions in $U$.

If $f$ is a real function defined in $\mathbb{H}^{n}$, we denote by ${ }^{\mathrm{v}} f$ the function defined by ${ }^{\mathrm{v}} f(p):=$ $f\left(p^{-1}\right)$, and, if $T \in \mathcal{D}^{\prime}\left(\mathbb{H}^{n}\right)$, then ${ }^{\mathrm{v}} T$ is the distribution defined by $\left\langle{ }^{\mathrm{v}} T \mid \phi\right\rangle:=\left\langle T \mid{ }^{\mathrm{v}} \phi\right\rangle$ for any test function $\phi$.

Following e.g., [21, p. 15], we can define a group convolution in $\mathbb{H}^{n}$ : if, for instance, $f \in \mathcal{D}\left(\mathbb{H}^{n}\right)$ and $g \in L_{\text {loc }}^{1}\left(\mathbb{H}^{n}\right)$, we set

$$
f * g(p):=\int f(q) g\left(q^{-1} \cdot p\right) d q \quad \text { for } q \in \mathbb{H}^{n} .
$$

We recall that if (say) $g$ is a smooth function and $P$ is a left-invariant differential operator, then

$$
P(f * g)=f * P g .
$$

We recall also that the convolution is again well defined when $f, g \in \mathcal{D}^{\prime}\left(\mathbb{H}^{n}\right)$, provided at least one of them has compact support. In this case, the following identities hold,

$$
\langle f * g \mid \phi\rangle=\left\langle\left. g\right|^{\mathrm{v}} f * \phi\right\rangle \text { and }\langle f * g \mid \phi\rangle=\left\langle f \mid \phi *^{\mathrm{v}} g\right\rangle,
$$

for any test function $\phi$.

As in [21], we also adopt the following multi-index notation for higher-order derivatives. If $I=\left(i_{1}, \ldots, i_{2 n+1}\right)$ is a multi-index, we set

$$
W^{I}=W_{1}^{i_{1}} \cdots W_{2 n}^{i_{2 n}} T^{i_{2 n+1}}
$$

By the Poincaré-Birkhoff-Witt theorem, the differential operators $W^{I}$ form a basis for the algebra of left-invariant differential operators in $\mathbb{H}^{n}$. Furthermore, we set $|I|:=i_{1}+\cdots+$ $i_{2 n}+i_{2 n+1}$ the order of the differential operator $W^{I}$, and $d(I):=i_{1}+\cdots+i_{2 n}+2 i_{2 n+1}$ its degree of homogeneity with respect to group dilations. 
Suppose now $f, g \in \mathcal{D}^{\prime}\left(\mathbb{H}^{n}\right)$ with $f$ compactly supported. Then, if $\psi \in \mathcal{D}\left(\mathbb{H}^{n}\right)$, we have

$$
\begin{aligned}
\left\langle\left(W^{I} f\right) * g \mid \psi\right\rangle & =\left\langle W^{I} f \mid \psi *{ }^{\mathrm{v}} g\right\rangle=(-1)^{|I|}\left\langle f \mid \psi *\left(W^{I}{ }^{\mathrm{v}} g\right)\right\rangle \\
& =(-1)^{|I|}\left\langle f *{ }^{\mathrm{v}} W^{I} \mathrm{v} g \mid \psi\right\rangle .
\end{aligned}
$$

Definition 3.1. Let $\omega \in \mathcal{D}\left(\mathbb{H}^{n}\right)$ be supported in the unit ball $B(e, 1)$, and assume $\int \omega(x) d x=1$. If $\varepsilon>0$, we denote by $\omega_{\varepsilon}$ the Friedrichs mollifier $\omega_{\varepsilon}(x):=\varepsilon^{-Q_{\omega}}\left(\delta_{1 / \varepsilon} x\right)$.

The procedure of regularization by convolution can be extended componentwise to differential forms in $L_{\text {loc }}^{1}\left(\mathbb{H}^{n}, E_{0}^{\bullet}\right)$, as follows: if $\alpha=\sum_{j} \alpha_{j} \xi_{j}^{h}$, we set

$$
\omega_{\varepsilon} * \alpha:=\sum_{j}\left(\omega_{\varepsilon} * \alpha_{j}\right) \xi_{j}^{h} .
$$

As above, denote by $*$ the group convolution in $\mathbb{H}^{n}$. By [21] and (23), if $u \in L_{\text {loc }}^{1}\left(\mathbb{H}^{n}\right)$, the convolution $u_{\varepsilon}:=u * \omega_{\varepsilon}$ enjoys the same properties of the usual regularizing convolutions in Euclidean spaces.

Following [20], we recall now the notion of kernel of type $\mu$.

Definition 3.2. A kernel of type $\mu$ is a homogeneous distribution of degree $\mu-Q$ (with respect to group dilations $\delta_{r}$ ), which is smooth outside of the origin.

The convolution operator with a kernel of type $\mu$ is called an operator of type $\mu$.

Proposition 3.3. Let $K \in \mathcal{D}^{\prime}\left(\mathbb{H}^{n}\right)$ be a kernel of type $\mu$.

(i) ${ }^{\mathrm{v}} K$ is again a kernel of type $\mu$.

(ii) $W K$ and $K W$ are associated with kernels of type $\mu-1$ for any horizontal derivative $W$.

(iii) If $\mu>0$, then $K \in L_{\mathrm{loc}}^{1}\left(\mathbb{H}^{n}\right)$.

Theorem 3.4. Suppose $0<\alpha<Q$, and let $K$ be a kernel of type $\alpha$. Then we have the following:

(i) If $1<p<Q / \alpha$, and $1 / q:=1 / p-\alpha / Q$, then there exists $C=C(p, \alpha)>0$ such that

$$
\|u * K\|_{L^{q}\left(\mathbb{H}^{n}\right)} \leqslant C\|u\|_{L^{p}\left(\mathbb{H}^{n}\right)}
$$

for all $u \in L^{p}\left(\mathbb{H}^{n}\right)$.

(ii) If $p \geqslant Q / \alpha$ and $B, B^{\prime} \subset \mathbb{H}^{n}$ are fixed balls with $B \subset B^{\prime}$, then for any $q \geqslant p$, there exists $C=C\left(B, B^{\prime}, p, q, \alpha\right)>0$

$$
\|u * K\|_{L^{q}\left(B^{\prime}\right)} \leqslant C\|u\|_{L^{p}(B)}
$$

for all $u \in L^{p}\left(\mathbb{H}^{n}\right)$ with supp $u \subset B$.

(iii) If $K$ is a kernel of type 0 and $1<p<\infty$, then there exists $C=C(p)>0$ such that

$$
\|u * K\|_{L^{p}\left(\mathbb{H}^{n}\right)} \leqslant C\|u\|_{L^{p}\left(\mathbb{H}^{n}\right)} .
$$


Proof. For statements (i) and (iii), we refer the reader to [20, Propositions 1.11 and 1.9]. As for (ii), if $p \geqslant Q / \alpha$, we choose $1<\tilde{p}<Q / \alpha$ such that $1 / \tilde{p} \leqslant 1 / q+\alpha / Q$. If we set $1 / \tilde{q}:=1 / \tilde{p}-\alpha / Q<1 / q$, then

$$
\begin{aligned}
& \|u * K\|_{L^{q}\left(B^{\prime}\right)} \leqslant C_{B^{\prime}}\|u * K\|_{L^{\tilde{q}}\left(B^{\prime}\right)} \leqslant C_{B^{\prime}}\|u * K\|_{L^{\tilde{q}}\left(\mathbb{H}^{n}\right)} \\
& \quad \leqslant C^{\prime}\left(B^{\prime}\right)\|u\|_{L^{\tilde{p}}\left(\mathbb{H}^{n}\right)} \leqslant C^{\prime}\left(B, B^{\prime}\right)\|u\|_{L^{p}(B) .}
\end{aligned}
$$

Lemma 3.5. Suppose $0<\alpha<Q$. If $K$ is a kernel of type $\alpha$ and $\psi \in \mathcal{D}\left(\mathbb{H}^{n}\right), \psi \equiv 1$, in a neighborhood of the origin, then statements (i) and (ii) of Theorem 3.4 still hold if we replace $K$ by $(1-\psi) K$.

Analogously, if $K$ is a kernel of type 0 and $\psi \in \mathcal{D}\left(\mathbb{H}^{n}\right)$, then statement (iii) of Theorem 3.4 still holds if we replace $K$ by $(\psi-1) K$.

Proof. As in [20, Proposition 1.11], we merely need note that $|(1-\psi) K(x)| \leqslant C_{\psi}|x|^{\alpha-Q}$, so that $(1-\psi) K \in L^{Q /(Q-\alpha), \infty}\left(\mathbb{H}^{n}\right)$, and therefore (i) and (ii) hold true.

Suppose now $\alpha=0$.

Note that $(\psi-1) K \in L^{1, \infty}\left(\mathbb{H}^{n}\right)$, and therefore also $u \rightarrow((\psi-1) K) * u$ is $L^{p}-L^{p}$ continuous by the Hausdorff-Young theorem. This proves that (iii) holds true.

Remark 3.6. By Theorem 3.4, Lemma 3.5 still holds if we replace $(1-\psi) K$ by $\psi K$.

The following estimate will be useful in the sequel.

Lemma 3.7. Let $g$ be a kernel of type $\mu>0$. Then, if $f \in \mathcal{D}\left(\mathbb{H}^{n}\right)$ and $R$ is a homogeneous polynomial of degree $\ell \geqslant 0$ in the horizontal derivatives, we have

$$
R(f * g)(p)=O\left(|p|^{\mu-Q-\ell}\right) \quad \text { as } p \rightarrow \infty .
$$

In addition, let $g$ be a smooth function in $\mathbb{H}^{n} \backslash\{0\}$ satisfying the logarithmic estimate

$$
|g(p)| \leqslant C(1+|\ln | p||),
$$

and suppose its horizontal derivatives are kernels of type $Q-1$ with respect to group dilations. Then, if $f \in \mathcal{D}\left(\mathbb{H}^{n}\right)$ and $R$ is a homogeneous polynomial of degree $\ell \geqslant 0$ in the horizontal derivatives, we have

$$
\begin{aligned}
& R(f * g)(p)=O\left(|p|^{-\ell}\right) \quad \text { as } p \rightarrow \infty \text { if } \ell>0 \\
& R(f * g)(p)=O(\ln |p|) \quad \text { as } p \rightarrow \infty \text { if } \ell=0 .
\end{aligned}
$$

Proof. The first part of the lemma is a particular instance of [21, Lemma 6.4]. As for the second part, we can repeat the same argument. Indeed, the first statement follows straightforwardly from the first part of the lemma since, by the Poincaré-Birkhoff-Witt theorem, we can write

$$
R(f * g)=\sum_{j} R_{\ell}^{\prime}\left(f * W_{j} g\right),
$$

where the differential operators $R_{j}^{\prime}$ have homogeneous degree $\ell-1$. Finally, the last statement can be proved as follows: suppose supp $f \subset B(0, M), M>1$, and 
take $|p|>2 M$. Then, keeping in mind that $1<M \leqslant\left|q^{-1} p\right| \leqslant M+|p|$,

$$
\begin{aligned}
|R(f * g)(p)| & \leqslant \int|f(q)|\left|g\left(q^{-1} p\right)\right| d q \leqslant C \int|f(q)|\left(1+\ln \left|q^{-1} p\right|\right) d q \\
& \leqslant C \int|f(q)|(1+\ln (M+|p|)) d q \leqslant C^{\prime}(1+\ln |p|) .
\end{aligned}
$$

\subsection{Rumin's Laplacians}

In this section, we recall the main properties of Rumin's generalization of the Laplace operator in Heisenberg groups. In order to introduce this operator, we need preliminarily the following property about the $L^{2}$-adjoint of Rumin's exterior differential $d_{c}$.

Proposition 3.8. Denote by $\delta_{c}=d_{c}^{*}$ the formal adjoint of $d_{c}$ in $L^{2}\left(\mathbb{H}^{n}, E_{0}^{*}\right)$. Then $\delta_{c}=$ $(-1)^{h} * d_{c} *$ on $E_{0}^{h}$.

Definition 3.9. In $\mathbb{H}^{n}$, following [50], we define the operator $\Delta_{\mathbb{H}, h}$ on $E_{0}^{h}$ by setting

$$
\Delta_{\mathbb{H}, h}= \begin{cases}d_{c} \delta_{c}+\delta_{c} d_{c} & \text { if } h \neq n, n+1 \\ \left(d_{c} \delta_{c}\right)^{2}+\delta_{c} d_{c} & \text { if } h=n \\ d_{c} \delta_{c}+\left(\delta_{c} d_{c}\right)^{2} & \text { if } h=n+1\end{cases}
$$

For the sake of simplicity, since a basis of $E_{0}^{h}$ is fixed, any $\alpha \in E_{0}^{h}$ can be identified with the vector $\left(\alpha_{1}, \ldots, \alpha_{N_{h}}\right)$ of its components, and the operator $\Delta_{\mathbb{H}, h}$ can be identified with a matrix-valued map, still denoted by $\Delta_{\mathbb{H}, h}$,

$$
\Delta_{\mathbb{H}, h}=\left(\Delta_{\mathbb{H}, h}^{i j}\right)_{i, j=1, \ldots, N_{h}}: \mathcal{D}^{\prime}\left(\mathbb{H}^{n}, \mathbb{R}^{N_{h}}\right) \rightarrow \mathcal{D}^{\prime}\left(\mathbb{H}^{n}, \mathbb{R}^{N_{h}}\right),
$$

where $N_{h}$ is the dimension of $E_{0}^{h}\left(N_{h}\right.$ is explicit in Theorem 2.5, (iv)) and $\mathcal{D}^{\prime}\left(\mathbb{H}^{n}, \mathbb{R}^{N_{h}}\right)$ is the space of vector-valued distributions on $\mathbb{H}^{n}$.

This identification makes it possible to avoid the notion of currents. We refer the reader to [8] for a more elegant presentation.

Remark 3.10. We stress that $\Delta_{\mathbb{H}, h}$ is a left-invariant differential operator of order 2 if $h \neq n, n+1$ and of order 4 if $h=n, n+1$ with respect to group dilations (see (9)), i.e., its components $\Delta_{\mathbb{H}, h}^{i j}$ can be written, with the notations of (22), as

$$
\Delta_{\mathbb{H}, h}^{i j}=\sum_{I} c_{I}^{i j} W^{I}
$$

with $d(I)=2$ if $h \neq n, n+1$ and $d(I)=4$ if $h=n, n+1$. In addition, $-\Delta_{\mathbb{H}, 0=}$ $\sum_{j=1}^{2 n}\left(W_{j}^{2}\right)$ is the usual sub-Laplacian of $\mathbb{H}^{n}$.

Remark 3.11. As a straightforward consequence of Proposition 3.8 for any $0 \leqslant h \leqslant$ $2 n+1$, we have $* \Delta_{\mathbb{H}, h}=\Delta_{\mathbb{H}, 2 n+1-h} *($ since $(2 n+1)$ is odd $)$.

It is proved in [50] that $\Delta_{\mathbb{H}, h}$ is hypoelliptic and maximal hypoelliptic in the sense of [32]. In general, if $\mathcal{L}$ is a differential operator on $\mathcal{D}^{\prime}\left(\mathbb{H}^{n}, \mathbb{R}^{N_{h}}\right)$, then $\mathcal{L}$ is said to be hypoelliptic 
if for any open set $\mathcal{V} \subset \mathbb{H}^{n}$ where $\mathcal{L} \alpha$ is smooth, then $\alpha$ is smooth in $\mathcal{V}$. In addition, if $\mathcal{L}$ is homogeneous of degree $a \in \mathbb{N}$, we say that $\mathcal{L}$ is maximal hypoelliptic if for any $\delta>0$, there exists $C=C(\delta)>0$ such that for any homogeneous polynomial $P$ in $W_{1}, \ldots, W_{2 n}$ of degree $a$, we have

$$
\|P \alpha\|_{L^{2}\left(\mathbb{H}^{n}, \mathbb{R}^{\left.N_{h}\right)}\right.} \leqslant C\left(\|\mathcal{L} \alpha\|_{L^{2}\left(\mathbb{H}^{n}, \mathbb{R}^{\left.N_{h}\right)}\right.}+\|\alpha\|_{L^{2}\left(\mathbb{H}^{n}, \mathbb{R}^{\left.N_{h}\right)}\right.}\right)
$$

for any $\alpha \in \mathcal{D}\left(B(0, \delta), \mathbb{R}^{N_{h}}\right)$.

The next theorem provides a key tool for the present paper: the existence of a suitable 'inverse' $\Delta_{\mathbb{H}, h}^{-1}$ of $\Delta_{\mathbb{H}, h}$ that is associated with a vector-valued kernel, which we still denote by $\Delta_{\mathbb{H}, h}^{-1}$.

Combining [50, Section 3] and [10, Theorems 3.1 and 4.1], we obtain the following result. We stress again the fact that the order of $\Delta_{\mathbb{H}, h}$ with respect to group dilations is 2 if $h \neq n, n+1$ whereas it is 4 if $h=n, n+1$.

Theorem 3.12 (See [3, Theorem 4.6]). If $0 \leqslant h \leqslant 2 n+1$, denote by a the order of $\Delta_{\mathbb{H}, h}$ with respect to group dilations (by Remark 3.10, $a=2$ if $h \neq n, n+1$ and $a=4$ if $h=n$, $n+1)$. Then there exists

$$
K_{i j} \in \mathcal{D}^{\prime}\left(\mathbb{H}^{n}\right) \cap \mathcal{C}^{\infty}\left(\mathbb{H}^{n} \backslash\{0\}\right) \quad \text { for } i, j=1, \ldots, N_{h},
$$

with the following properties:

(i) If $a<Q$, then the $K_{i j}$ 's are kernels of type $a$, for $i, j=1, \ldots, N_{h}$. If $a=Q$, then the $K_{i j}$ 's satisfy the logarithmic estimate $\left|K_{i j}(p)\right| \leqslant C(1+|\ln \rho(p)|)$ and hence belong to $L_{\text {loc }}^{1}\left(\mathbb{H}^{n}\right)$. Moreover, their horizontal derivatives $W_{\ell} K_{i j}, \ell=1, \ldots, 2 n$, are kernels of type $Q-1$.

(ii) When $\alpha=\left(\alpha_{1}, \ldots, \alpha_{N_{h}}\right) \in \mathcal{D}\left(\mathbb{H}^{n}, \mathbb{R}^{N_{h}}\right)$ (here again $\left.N_{h}=\operatorname{dim} E_{0}^{h}\right)$, if we set

$$
\Delta_{\mathbb{H}, h}^{-1} \alpha:=\left(\sum_{j} \alpha_{j} * K_{1 j}, \ldots, \sum_{j} \alpha_{j} * K_{N_{h} j}\right),
$$

then

$$
\Delta_{\mathbb{H}, h} \Delta_{\mathbb{H}, h}^{-1} \alpha=\alpha
$$

Moreover, if $a<Q$, also

$$
\Delta_{\mathbb{H}, h}^{-1} \Delta_{\mathbb{H}, h} \alpha=\alpha .
$$

(iii) If $a=Q$, then for any $\alpha \in \mathcal{D}\left(\mathbb{H}^{n}, \mathbb{R}^{N_{h}}\right)$, there exists $\beta_{\alpha}:=\left(\beta_{1}, \ldots, \beta_{N_{h}}\right) \in \mathbb{R}^{N_{h}}$ such that

$$
\Delta_{\mathbb{H}, h}^{-1} \Delta_{\mathbb{H}, h} \alpha-\alpha=\beta_{\alpha}
$$

Remark 3.13. Coherently with formula (24), the matrix-valued operator $\Delta_{\mathbb{H}, h}^{-1}$ can be identified with an operator (still denoted by $\Delta_{\mathbb{H}, h}^{-1}$ ) acting on smooth compactly supported differential forms in $\mathcal{D}\left(\mathbb{H}^{n}, E_{0}^{h}\right)$. Moreover, when the notation will not be misleading, we shall denote by $\Delta_{\mathbb{H}, h}^{-1}$ its kernel. 
The following lemma states that $d_{c}$ and $\Delta_{\mathbb{H}}$ commute.

Lemma 3.14. We note that the Laplace operator commutes with the exterior differential $d_{c}$. More precisely, if $\alpha \in \mathcal{C}^{\infty}\left(\mathbb{H}^{n}, E_{0}^{h}\right)$ and $n \geqslant 1$,

(i) $d_{c} \Delta_{\mathbb{H}, h} \alpha=\Delta_{\mathbb{H}, h+1} d_{c} \alpha, h=0,1, \ldots, 2 n, h \neq n-1, n$,

(ii) $d_{c} \delta_{c} d_{c} \Delta_{\mathbb{H}, n-1} \alpha=\Delta_{\mathbb{H}, n} d_{c} \alpha(h=n-1)$,

(iii) $d_{c} \Delta_{\mathbb{H}, n} \alpha=d_{c} \delta_{c} \Delta_{\mathbb{H}, n+1} d_{c} \alpha(h=n)$,

(iv) $d_{c} \delta_{c} \Delta_{\mathbb{H}, n} \alpha=\Delta_{\mathbb{H}, n} d_{c} \delta_{c} \alpha(h=n)$.

Proof. The proof is an easy consequence of the fact that $d_{c}^{2}=0$. Indeed, let us prove (i). Since $h \neq n-1, n$, we write $d_{c} \Delta_{\mathbb{H}, h} \alpha=d_{c}\left(d_{c} \delta_{c}+\delta_{c} d_{c}\right) \alpha=d_{c}^{2} \delta_{c} \alpha+d_{c} \delta_{c} d_{c} \alpha=d_{c} \delta_{c} d_{c} \alpha=$ $d_{c} \delta_{c} d_{c} \alpha+\delta_{c} d_{c}^{2} \alpha=\left(d_{c} \delta_{c}+\delta_{c} d_{c}\right) d_{c} \alpha=\Delta_{\mathbb{H}, h+1} d_{c} \alpha$.

To prove (ii), we write $d_{c} \delta_{c} d_{c} \Delta_{\mathbb{H}, n-1} \alpha=d_{c} \delta_{c} d_{c}\left(d_{c} \delta_{c}+\delta_{c} d_{c}\right) \alpha=d_{c} \delta_{c} d_{c}^{2} \delta_{c} \alpha+d_{c} \delta_{c} d_{c} \delta_{c} d_{c} \alpha$ $=\left(d_{c} \delta_{c}\right)^{2} d_{c} \alpha=\left(d_{c} \delta_{c}\right)^{2} d_{c} \alpha+\left(\delta_{c} d_{c}\right) d_{c} \alpha=\Delta_{\mathbb{H}, n} d_{c} \alpha$. An analogous argument applies to (iii) and (iv).

The commutation of $d_{c}$ and $\delta_{c}$ with $\Delta_{\mathbb{H}}^{-1}$ follows from the previous lemma.

Lemma 3.15. If $\alpha \in \mathcal{D}\left(\mathbb{H}^{n}, E_{0}^{h}\right)$ and $n \geqslant 1$,

(i) $d_{c} \Delta_{\mathbb{H}, h}^{-1} \alpha=\Delta_{\mathbb{H}, h+1}^{-1} d_{c} \alpha, h=0,1, \ldots, 2 n, h \neq n-1, n+1$,

(ii) $d_{c} \Delta_{\mathbb{H}, n-1}^{-1} \alpha=d_{c} \delta_{c} \Delta_{\mathbb{H}, n}^{-1} d_{c} \alpha(h=n-1)$,

(iii) $d_{c} \delta_{c} d_{c} \Delta_{\mathbb{H}, n+1}^{-1} \alpha=\Delta_{\mathbb{H}, n+2}^{-1} d_{c} \alpha(h=n+1)$,

(iv) $\delta_{c} \Delta_{\mathbb{H}, h}^{-1} \alpha=\Delta_{\mathbb{H}, h-1}^{-1} \delta_{c} \alpha h=1, \ldots, 2 n+1, h \neq n, n+2$,

(v) $\delta_{c} \Delta_{\mathbb{H}, n+2}^{-1} \alpha=\delta_{c} d_{c} \Delta_{\mathbb{H}, n+1}^{-1} \delta_{c} \alpha(h=n+2)$,

(vi) $\delta_{c} d_{c} \delta_{c} \Delta_{\mathbb{H}, n}^{-1} \alpha=\Delta_{\mathbb{H}, n-1}^{-1} \delta_{c} \alpha(h=n)$.

Proof. Let us prove (i)-(iii). Put

$$
\begin{aligned}
\omega_{h} & :=d_{c} \Delta_{\mathbb{H}, h}^{-1} \alpha-\Delta_{\mathbb{H}, h+1}^{-1} d_{c} \alpha \quad \text { if } h \neq n-1, n+1, \\
\omega_{n-1} & :=d_{c} \Delta_{\mathbb{H}, n-1}^{-1} \alpha-d_{c} \delta_{c} \Delta_{\mathbb{H}, n}^{-1} d_{c} \alpha, \\
\omega_{n+1} & :=d_{c} \delta_{c} d_{c} \Delta_{\mathbb{H}, n+1}^{-1} \alpha-\Delta_{\mathbb{H}, n+2}^{-1} d_{c} \alpha .
\end{aligned}
$$

We note first that, by Theorem 3.12 and Proposition 3.3 , for all $h=1, \ldots, 2 n, \omega_{h}=$ $M_{h} * \alpha$, where $M_{h}$ is a kernel of type 1 . Thus, by Lemma 3.7,

$$
\omega_{h}(x)=O\left(|x|^{1-Q}\right) \text { as } x \rightarrow \infty .
$$

We claim that

$$
\Delta_{\mathbb{H}, h+1} \omega_{h}=0 \quad \text { for } h=1, \ldots, 2 n .
$$

Thus, by [10, Proposition 3.2], $\omega_{h}$ is a form with polynomial coefficients. Then, by (27), necessarily $\omega_{h} \equiv 0$. Thus we have proved (i)-(iii).

We are left then with the proof of claim (28). 
Suppose first $h \neq n-1, n, n+1$. By Lemma 3.14(i) and by Theorem 3.12, we have

$$
\begin{aligned}
\Delta_{\mathbb{H}, h+1} \omega_{h} & =\Delta_{\mathbb{H}, h+1} d_{c} \Delta_{\mathbb{H}, h}^{-1} \alpha-\Delta_{\mathbb{H}, h+1} \Delta_{\mathbb{H}, h+1}^{-1} d_{c} \alpha \\
& =d_{c} \Delta_{\mathbb{H}, h} \Delta_{\mathbb{H}, h}^{-1} \alpha-d_{c} \alpha=0 .
\end{aligned}
$$

If $h=n-1$, then by Lemma 3.14(iii), (iv) and by Theorem 3.12,

$$
\begin{aligned}
\Delta_{\mathbb{H}, n} \omega_{n-1} & =\Delta_{\mathbb{H}, n}\left(d_{c} \Delta_{\mathbb{H}, n-1}^{-1} \alpha-d_{c} \delta_{c} \Delta_{\mathbb{H}, n}^{-1} d_{c} \alpha\right) \\
& =d_{c} \delta_{c} d_{c} \Delta_{\mathbb{H}, n-1} \Delta_{\mathbb{H}, n-1}^{-1} \alpha-d_{c} \delta_{c} \Delta_{\mathbb{H}, n} \Delta_{\mathbb{H}, n}^{-1} d_{c} \alpha=0 .
\end{aligned}
$$

If $h=n$, then (keeping in mind that $d_{c} \Delta_{\mathbb{H}, n}^{-1} \alpha$ is a form of degree $n+1$ and $\Delta_{\mathbb{H}, n}^{-1} \alpha$ is a form of degree $n$, we use again Lemma 3.14(i))

$$
\begin{aligned}
\Delta_{\mathbb{H}, n+1} \omega_{n} & =\Delta_{\mathbb{H}, n+1}\left(d_{c} \Delta_{\mathbb{H}, n}^{-1} \alpha-\Delta_{\mathbb{H}, n+1}^{-1} d_{c} \alpha\right) \\
& =d_{c} \Delta_{\mathbb{H}, n} \Delta_{\mathbb{H}, n}^{-1} \alpha-d_{c} \alpha=0 .
\end{aligned}
$$

Finally, if $h=n+1$, then again from Lemma 3.14(i),

$$
\begin{aligned}
\Delta_{\mathbb{H}, n+2} \omega_{n+1} & =\Delta_{\mathbb{H}, n+2}\left(d_{c} \Delta_{\mathbb{H}, n+1}^{-1} \alpha-\Delta_{\mathbb{H}, n+2}^{-1} d_{c} \alpha\right) \\
& =d_{c} \Delta_{\mathbb{H}, n+1} \Delta_{\mathbb{H}, n+1}^{-1} \alpha-d_{c} \alpha=0 .
\end{aligned}
$$

This proves (28), and hence we have proved (i)-(iii).

Since $\delta_{c}=(-1)^{h} * d_{c} *$, and keeping in mind Remark 3.11, the remaining assertions (iv)-(vi) follow by the Hodge duality from (i)-(iii).

\section{Function spaces}

\subsection{Sobolev spaces on Heisenberg groups}

Let $U \subset \mathbb{H}^{n}$ be an open set and let $1 \leqslant p \leqslant \infty$ and $m \in \mathbb{N} ; W_{\text {Euc }}^{m, p}(U)$ denotes the usual Sobolev space. We want now to introduce intrinsic (horizontal) Sobolev spaces.

Since here we are dealing only with integer order Folland-Stein function spaces, we can give this simpler definition (for a general presentation, see e.g., [20]).

Definition 4.1. If $U \subset \mathbb{H}^{n}$ is an open set, $1 \leqslant p \leqslant \infty$ and $m \in \mathbb{N}$, then the space $W^{m, p}(U)$ is the space of all $u \in L^{p}(U)$ such that, with the notation of (22),

$$
W^{I} u \in L^{p}(U) \text { for all multi-indices } I \text { with } d(I) \leqslant m,
$$

endowed with the natural norm, which we denote by

$$
\|u\|_{W^{k, p}(U)}:=\sum_{d(I) \leqslant m}\left\|W^{I} u\right\|_{L^{p}(U)} .
$$

Folland-Stein Sobolev spaces enjoy the following properties akin to those of the usual Euclidean Sobolev spaces (see [20] and, e.g., [25]).

Theorem 4.2. If $U \subset \mathbb{H}^{n}, 1 \leqslant p<\infty$, and $k \in \mathbb{N}$, then

(i) $W^{k, p}(U)$ is a Banach space; 
(ii) $W^{k, p}(U) \cap C^{\infty}(U)$ is dense in $W^{k, p}(U)$;

(iii) if $U=\mathbb{H}^{n}$, then $\mathcal{D}\left(\mathbb{H}^{n}\right)$ is dense in $W^{k, p}(U)$.

Definition 4.3. If $U \subset \mathbb{H}^{n}$ is open and if $1 \leqslant p<\infty$, we denote by $\stackrel{\circ}{W}^{k, p}(U)$ the completion of $\mathcal{D}(U)$ in $W^{k, p}(U)$.

Remark 4.4. If $U \subset \mathbb{H}^{n}$ is bounded, by the (iterated) Poincaré inequality (see e.g., [34]), it follows that the norms

$$
\|u\|_{W^{k, p}(U)} \quad \text { and } \quad \sum_{d(I)=k}\left\|W^{I} u\right\|_{L^{p}(U)}
$$

are equivalent on $\stackrel{\circ}{W}^{k, p}(U)$ when $1 \leqslant p<\infty$.

If $U \subset \mathbb{H}^{n}$ is an open set and $1<p<\infty, W^{-k, p}(U)$ is the dual space of $\stackrel{\circ}{W}^{k, p^{\prime}}(U)$, where $1 / p+1 / p^{\prime}=1$.

Remark 4.5. It is well known that

$$
\begin{aligned}
& W^{-k, p}(U) \\
& =\left\{f_{0}+\sum_{d(I)=k} W^{I} f_{I}: f_{0}, f_{I} \in L^{p}(U) \text { for any multi-index } I \text { such that } d(I)=k\right\}
\end{aligned}
$$

and

$$
\|u\|_{W^{-k, p}(U)} \approx \inf \left\{\left\|f_{0}\right\|_{L^{p}(U)}+\sum_{I}\left\|f_{I}\right\|_{L^{p}(U)}: d(I)=k, f_{0}+\sum_{d(I)=k} W^{I} f_{I}=u\right\} .
$$

If $U$ is bounded, then we can take $f_{0}=0$.

Finally, we stress that

$$
\left\{f_{0}+\sum_{d(I)=k} W^{I} f_{I}, f_{0}, f_{I} \in \mathcal{D}(U) \text { for any multi-index } I \text { such that } d(I)=k\right\}
$$

is dense in $W^{-k, p}(U)$.

Definition 4.6. If $U \subset \mathbb{H}^{n}$ is an open set, $0 \leqslant h \leqslant 2 n+1,1 \leqslant p \leqslant \infty$ and $m \geqslant 0$, we denote by $W^{m, p}\left(U, \bigwedge^{h} \mathfrak{h}\right)$ (by $\left.\stackrel{\circ}{W}^{m, p}\left(U, \bigwedge^{h} \mathfrak{h}\right)\right)$ the space of all sections of $\bigwedge^{h} \mathfrak{h}$ such that their components with respect to a given left-invariant frame belong to $W^{m, p}(U)$ (to $\stackrel{\circ}{W}^{m, p}(U)$, respectively), endowed with its natural norm.

The spaces $W^{m, p}\left(U, E_{0}^{h}\right)$ and $\stackrel{\circ}{W}^{m, p}\left(U, E_{0}^{h}\right)$ are defined in the same way. In addition,

$$
W^{-m, p}\left(U, E_{0}^{h}\right):=\left(\stackrel{\circ}{W^{m}, p^{\prime}}\left(U, E_{0}^{h}\right)\right)^{*} .
$$


Clearly, these definitions are independent of the choice of the frame itself.

On the other hand, the spaces $W^{-m, p}\left(U, E_{0}^{h}\right)$ can be viewed as spaces of currents on $\left(E_{0}^{\bullet}, d_{c}\right)$ as in [8, Proposition 3.14]. More precisely, we have the following remark.

Remark 4.7. As in [8, Proposition 3.14], an element of $W^{-m, p}\left(U, E_{0}^{h}\right)$ can be identified (with respect to our basis) with an $N_{h}$-tuple

$$
\left(T_{1}, \ldots, T_{N_{h}}\right) \in\left(W^{-m, p}\left(U, E_{0}^{h}\right)\right)^{N_{h}} .
$$

This is nothing but the intuitive notion of 'currents as differential forms with distributional coefficients'. The action of $u \in W^{-m, p}\left(U, E_{0}^{h}\right)$ associated with $\left(T_{1}, \ldots, T_{N_{h}}\right)$ on the form $\sum_{j} \alpha_{j} \xi_{j}^{h} \in \stackrel{\circ}{W^{m}}, p^{\prime}\left(U, E_{0}^{h}\right)$ is given by

$$
\langle u \mid \alpha\rangle:=\sum_{j}\left\langle T_{j} \mid \alpha_{j}\right\rangle .
$$

On the other hand, suppose for the sake of simplicity that $U$ is bounded. Then by Remark 4.5, there exist $f_{I}^{j} \in L^{p}(U), j=1, \ldots, N_{h}, i=1, \ldots, 2 n+1$ such that

$$
\langle u \mid \alpha\rangle=\sum_{j} \sum_{d(I)=m} \int_{U} f_{I}^{j}(x) W^{I} \alpha_{j}(x) d x .
$$

Alternatively, one can express duality in spaces of differential forms using the pairing between $h$-forms and $(2 n+1-h)$-forms defined by

$$
(\alpha, \beta) \mapsto \int_{U} \alpha \wedge \beta
$$

Note that this makes sense for Rumin forms and is a non-degenerate pairing. In this manner, the dual of $L^{p}\left(U, E_{0}^{h}\right)$ is $L^{p^{\prime}}\left(U, E_{0}^{2 n+1-h}\right)$. Hence $W^{-m, p}\left(U, E_{0}^{h}\right)$ consists of differential forms of degree $2 n+1-h$ whose coefficients are distributions belonging to $W^{-m, p}(U)$.

In the Riemannian setting, Sobolev spaces of differential forms are invariant with respect to the pull-back operator associated with sufficiently smooth diffeomorphisms (see, e.g., [55, Lemma 1.3.9]). An analogous statement holds for Folland-Stein Sobolev spaces in Heisenberg groups, provided we restrict ourselves to contact diffeomorphisms. Indeed, we have the following lemma.

Lemma 4.8. If $k$ is a positive integer, let $U, V$ be open subsets of $\mathbb{H}^{n}$. Let $\phi: U \rightarrow V$ be a $C^{k}$-bounded contact diffeomorphism. Let $\ell=-k+1, \ldots, k-1$. Then the pull-back operator $\phi^{\sharp}$ from $W^{\ell, p}$ forms on $V$ to $W^{\ell, p}$ forms on $U$ is bounded, and its norm depends only on the $C^{k}$ norms of $\phi$ and $\phi^{-1}$.

Proof. When $\ell \geqslant 0$, this follows from the chain rule and the change of variables formula. According to the change of variables formula

$$
\int_{U} \phi^{\sharp} \alpha \wedge \phi^{\sharp} \beta=\int_{V} \alpha \wedge \beta,
$$

the adjoint of $\phi^{\sharp}$ with respect to the above pairing is $\left(\phi^{-1}\right)^{\sharp}$. Hence $\phi^{\sharp}$ is bounded on negative Sobolev spaces of differential forms as well. 


\subsection{Sobolev spaces on contact sub-Riemannian manifolds with bounded geometry}

First of all, let us give the definition of contact manifolds of bounded geometry.

Definition 4.9. Let $k$ be a positive integer and let $B(e, 1)$ denote the unit sub-Riemannian ball in $\mathbb{H}^{n}$. We say that a sub-Riemannian contact manifold $(M, H, g)$ has bounded $C^{k}$-geometry if there exist constants $r, C>0$ such that, for every $x \in M$, there exists a contactomorphism (i.e., a diffeomorphism preserving the contact forms) $\phi_{x}: B(e, 1) \rightarrow M$ that satisfies

(1) $B(x, r) \subset \phi_{x}(B(e, 1))$;

(2) $\phi_{x}$ is $C$-bi-Lipschitz, i.e.,

$$
\frac{1}{C} d(p, q) \leqslant d_{M}\left(\phi_{x}(p), \phi_{x}(q)\right) \leqslant C d(p, q) \text { for all } p, q \in B(e, 1) ;
$$

(3) coordinate changes $\phi_{x} \circ \phi_{y}^{-1}$ and their first $k$ derivatives with respect to unit left-invariant horizontal vector fields are bounded by $C$.

Remark 4.10. Compact sub-Riemannian contact manifolds have bounded geometry. More examples arise from covering spaces of such compact manifolds. Note that every orientable compact 3-manifold admits a contact structure [39], it can be equipped with sub-Riemannian structures and its universal covering space is usually non-compact. This leads to a large variety of non-compact bounded geometry sub-Riemannian contact 3-manifolds.

The following covering lemma is basically [40, Theorem 1.2].

Lemma 4.11. Let $(M, H, g)$ be a bounded $C^{k}$-geometry sub-Riemannian contact manifold, where $k$ is a positive integer. Then there exist $\rho>0$ (depending only on the radius $r$ of Definition 4.9) and an at most countable covering $\left\{B\left(x_{j}, \rho\right)\right\}$ of $M$ such that we have the following:

(i) Each ball $B\left(x_{j}, \rho\right)$ is contained in the image of one of the contact charts of Definition 4.9 .

(ii) $B\left(x_{j}, \frac{1}{5} \rho\right) \cap B\left(x_{i}, \frac{1}{5} \rho\right)=\emptyset$ if $i \neq j$.

(iii) The covering is uniformly locally finite. Even more, there exists an $N=N(M) \in \mathbb{N}$ such that for each ball $B(x, \rho)$,

$$
\#\left\{k \in \mathbb{N} \text { such that } B\left(x_{k}, \rho\right) \cap B(x, \rho) \neq \emptyset\right\} \leqslant N .
$$

In addition, if $B\left(x_{k}, \rho\right) \cap B(x, \rho) \neq \emptyset$, then $B\left(x_{k}, \rho\right) \subset B(x, r)$, where $B(x, r)$ has been defined in Definition 4.9(2).

Proof. First, we note that $M$ is separable. Indeed, let $x \in M$ be fixed. With the notations of Definition 4.9 , if we set $\phi_{x}(B(0,1)):=\mathcal{U}_{x}$, then $\left\{\mathcal{U}_{x}, x \in M\right\}$ is an open covering of $M$. Let now $\left\{\mathcal{V}_{x_{j}}, j \in \mathbb{N}\right\}$ be a countable refinement of $\left\{\mathcal{U}_{x}, x \in M\right\}$ (see [58, Lemma 1.9]). 
For any $j \in \mathbb{N}$, let $S_{j}$ be a countable dense subset of $\phi_{x_{j}}^{-1}\left(\mathcal{V}_{x_{j}}\right)$; then $\phi_{x_{j}}\left(S_{j}\right)$ is a countable dense subset of $\mathcal{V}_{x_{j}}$. Thus $\Sigma:=\bigcup_{j} \phi_{x_{j}}\left(S_{j}\right)$ is a countable dense subset of $M$.

Let now $\rho \in(0, r / 2)$ be fixed. Then, by [40, Theorems 1,2$]$, there exists a family of disjoint balls $\left\{B\left(x_{\alpha}, \frac{\rho}{5}\right)\right\}$ such that $\left\{B\left(x_{\alpha}, \rho\right)\right\}$ is an open covering of $M$. We prove now that we can extract a countable sub-family $\left\{B\left(x_{\alpha_{j}}, \rho\right)\right\}=:\left\{B\left(x_{j}, \rho\right)\right\}$, which is still an open covering of $M$. Indeed, for any $y \in \Sigma$, let us prove that $\#\left\{\alpha\right.$ such that $\left.y \in B\left(x_{\alpha}, \rho\right)\right\} \leqslant N$, where $N$ is a geometric constant. If $y \in B\left(x_{\alpha}, \rho\right) \cap B\left(x_{\beta}, \rho\right)$, then $d_{M}\left(x_{\alpha}, x_{\beta}\right)<2 \rho$. In addition, then $B\left(x_{\alpha}, \rho\right)$ and $B\left(x_{\beta}, \rho\right)$ are contained in $\phi_{y}(B(e, 1))$ since $2 \rho<r$. From now on, we assume $\rho>0$ is fixed with $3 \rho<r$. We note that by $(30)$,

$$
B\left(\phi_{y}^{-1}\left(x_{\alpha}\right), \rho / 5 C\right) \subset \phi_{y}^{-1}\left(B\left(x_{\alpha}, \rho / 5\right)\right)
$$

so that if $\alpha, \beta \in\left\{\alpha\right.$ such that $\left.y \in B\left(x_{\alpha}, \rho\right)\right\}$, then

$$
B\left(\phi_{y}^{-1}\left(x_{\alpha}\right), \rho / 5 C\right) \cap B\left(\phi_{y}^{-1}\left(x_{\beta}\right), \rho / 5 C\right)=\emptyset .
$$

By the doubling property of the Lebesgue measure in $\mathbb{H}^{n}$ with respect to Cygan-Korányi's balls, this is possible only for at most $N$ balls $B\left(\phi_{y}^{-1}\left(x_{\alpha}\right), \rho / 5 C\right)$, where $N=N(C, \rho)$. It follows that $\left\{B\left(x_{\alpha}, \rho\right)\right.$, such that $y \in B\left(x_{\alpha}, \rho\right)$ and $\left.y \in \Sigma\right\}$ is a countable sub-family of balls $\left\{B\left(x_{j}, \rho\right), j \in \mathbb{N}\right\} \subset\left\{B\left(x_{\alpha}, \rho\right)\right\}$ such that the balls $\left\{B\left(x_{j}, \rho / 5\right), j \in \mathbb{N}\right\}$ are disjoint. Finally, we note that by the density of $\Sigma$, straightforwardly $\left\{B\left(x_{j}, \rho\right), j \in \mathbb{N}\right\}$ still covers all $M$ since the balls have the same radius $\rho$.

Finally, note that our previous arguments yield also that the covering is uniformly locally finite. Indeed, let $x$ be fixed and let $B\left(x_{k}, \rho\right) \cap B(x, \rho) \neq \emptyset$. Then $B\left(x_{k}, \rho\right) \subset$ $B(x, 3 \rho) \subset B(x, r)$ since $3 \rho<r$. Consider now the family of open sets

$$
\mathcal{B}:=\left\{\phi_{x}^{-1}\left(B\left(x_{k}, \rho / 5\right)\right), \text { with } B\left(x_{k}, \rho\right) \cap B(x, \rho) \neq \emptyset\right\} .
$$

By definition, the open sets of $\mathcal{B}$ are disjoint (by (ii)), and their union is contained in $B(e, 1)$. In addition, again by $(30)$

$$
B\left(\phi_{x}^{-1}\left(x_{k}\right), \rho / 5 C\right) \subset \phi_{x}^{-1}\left(B\left(x_{k}, \rho / 5\right)\right),
$$

and the assertion follows again by a doubling argument in $\mathbb{H}^{n}$.

We can define now Sobolev spaces (involving a positive or negative number of derivatives) on bounded geometry contact sub-Riemannian manifolds.

Definition 4.12. Let $k$ be a positive integer, let $(M, H, g)$ be a bounded $C^{k}$-geometry sub-Riemannian contact manifold, and let $\left\{\chi_{j}\right\}$ be a partition of unity subordinate to the atlas $\mathcal{U}:=\left\{B\left(x_{j}, \rho\right), \phi_{x_{j}}\right\}$ of Lemma 4.11. From now on, for the sake of simplicity, we shall write $\phi_{j}:=\phi_{x_{j}}$. We stress that $\phi_{j}^{-1}\left(\operatorname{supp} \chi_{j}\right) \subset B(e, 1)$. If $\alpha$ is a Rumin differential form on $M$, we say that $\alpha \in W_{\mathcal{U}}^{\ell, p}\left(M, E_{0}^{\bullet}\right)$ for $\ell \in \mathbb{Z},-k+1 \leqslant \ell \leqslant k-1$ and $p \geqslant 1$ if

$$
\phi_{j}^{\#}\left(\chi_{j} \alpha\right) \in W^{\ell, p}\left(\mathbb{H}^{n}, E_{0}^{\bullet}\right) \quad \text { for } j \in \mathbb{N}
$$

(note that $\phi_{j}^{\#}\left(\chi_{j} \alpha\right)$ is compactly supported in $B(e, 1)$ and therefore can be continued by zero on all of $\left.\mathbb{H}^{n}\right)$. Then we set

$$
\|\alpha\|_{W_{\mathcal{U}}^{\ell, p}\left(M, E_{0}^{*}\right)}:=\left(\sum_{j}\left\|\phi_{j}^{\#}\left(\chi_{j} \alpha\right)\right\|_{W^{\ell, p}\left(\mathbb{H}^{n}, E_{0}^{*}\right)}^{p}\right)^{1 / p} .
$$


The following result shows that the definition of the Sobolev spaces $W_{\mathcal{U}}^{\ell, p}\left(M, E_{0}^{\bullet}\right)$ does not depend on the atlas $\mathcal{U}$. Therefore, once the proposition is proved, we drop the index $\mathcal{U}$ from the notation for Sobolev norms.

Proposition 4.13. Let $k$ and $\ell$ be as above, and let $(M, H, g)$ be a bounded $C^{k}$-geometry sub-Riemannian contact manifold. If $\mathcal{U}^{\prime}:=\left\{B\left(y_{j}, \rho^{\prime}\right), \phi_{y_{j}}^{\prime}\right\}$ is another atlas of $M$ satisfying Definition 4.9 and Lemma 4.11 with the same choice of $\rho$, and $\left\{\chi_{j}^{\prime}\right\}$ is an associated partition of unity, then

$$
W_{\mathcal{U}}^{\ell, p}\left(M, E_{0}^{\bullet}\right)=W_{\mathcal{U}^{\prime}}^{\ell, p}\left(M, E_{0}^{\bullet}\right),
$$

with equivalent norms.

Proof. Let $j \in \mathbb{N}$ be fixed, and let $\left(B\left(x_{j}, \rho\right), \phi_{j}\right)$ be a chart of $\mathcal{U}$. We can write

$$
\chi_{j}=\sum_{k \in I_{j}} \chi_{k}^{\prime} \chi_{j},
$$

where $\# I_{j} \leqslant N$, since, by Lemma 4.11 (iii), $B\left(x_{j}, \rho\right)$ is covered by at most $N$ balls of the covering associated with $\mathcal{U}^{\prime}$. Thus, by Definition $4.9(3)$ and keeping in mind that supp $\chi_{k}^{\prime} \subset B\left(x_{j}, r\right)($ since $3 \rho<r)$, we have

$$
\begin{aligned}
\left\|\phi_{j}^{\#}\left(\chi_{j} \alpha\right)\right\|_{W^{\ell, p}\left(\mathbb{H}^{n}, E_{0}^{\bullet}\right)} & \leqslant \sum_{k \in I_{j}}\left\|\phi_{j}^{\#}\left(\chi_{k}^{\prime} \chi_{j} \alpha\right)\right\|_{W^{\ell, p}\left(\mathbb{H}^{n}, E_{0}^{\bullet}\right)} \\
& \leqslant c \sum_{k \in I_{j}}\left\|\phi_{j}^{\#}\left(\chi_{k}^{\prime} \alpha\right)\right\|_{W^{\ell, p}\left(\mathbb{H}^{n}, E_{0}^{\bullet}\right)} \\
& =c \sum_{k \in I_{j}}\left\|\left(\phi_{j} \phi_{k}^{\prime-1}\right)^{\#} \phi_{k}^{\prime \#}\left(\chi_{k}^{\prime} \alpha\right)\right\|_{W^{\ell, p}\left(\mathbb{H}^{n}, E_{0}^{\bullet}\right)} \\
& \leqslant c \sum_{k \in I_{j}}\left\|\phi_{k}^{\prime \#}\left(\chi_{k}^{\prime} \alpha\right)\right\|_{W^{\ell, p}\left(\mathbb{H}^{n}, E_{0}^{\bullet}\right)} \\
& \leqslant c N\|\alpha\|_{W_{\mathcal{U}^{\prime}, p}^{\ell}\left(M, E_{0}^{\bullet}\right)}
\end{aligned}
$$

\section{Homotopy formulas and Poincaré and Sobolev inequalities}

In this paper, we are mainly interested to obtain functional inequalities for differential forms that are the counterparts of the classical $(p, q)$-Sobolev and Poincaré inequalities on a ball $B \subset \mathbb{R}^{n}$ with sharp exponents of the form

$$
\left\|u-u_{B}\right\|_{L^{q}}(B) \leqslant C(r)\|\nabla u\|_{L^{p}}(B)
$$

(as well as of its counterpart for compactly supported functions). In this case, we can choose $q=p n /(n-p)$, provided $p<n$.

Definition 5.1. Take $\lambda>1$ and set $B=B(e, 1)$ and $B^{\prime}=B(e, \lambda)$. If $1 \leqslant h \leqslant 2 n+1$ and $q \geqslant p \geqslant 1$, we say that the interior $\mathbb{H}$-Poincaré -P $_{q}$ inequality holds in $E_{0}^{h}$ if there exists a 
constant $C$ such that, for every $d_{c^{-}}$exact differential $k$-form $\omega$ in $L^{p}\left(B^{\prime} ; E_{0}^{h}\right)$ there exists a differential $(k-1)$-form $\phi$ in $L^{q}\left(B, E_{0}^{h-1}\right)$ such that $d_{c} \phi=\omega$ and

$$
\|\phi\|_{L^{q}\left(B, E_{0}^{h-1}\right)} \leqslant C\|\omega\|_{L^{p}\left(B^{\prime}, E_{0}^{h}\right)} \quad \text { interior } \mathbb{H}-\text { Poincaré } p, q(h) .
$$

Remark 5.2. As we pointed out in $\S 1.1$, what we call interior $\mathbb{H}$-Poincaré $p, q(h)$ is a slightly weaker formulation of the standard Poincaré inequality where $B=B^{\prime}$. The word 'interior' is meant to stress that the inequality is not affected by the geometry of the boundary of the ball. This is obtained thanks to a 'loss on domain', passing from a larger ball $B^{\prime}$ to a smaller ball $B$.

Remark 5.3. If $h=1$ and $Q>p \geqslant 1$, then ( $\mathbb{H}$-Poincaré ${ }_{p, q}(1)$ ) is nothing but the usual Poincaré inequality with $\frac{1}{p}-\frac{1}{q}=\frac{1}{Q}$ (see e.g., $[17,23,38]$ ).

Remark 5.4. If we replace the Rumin complex $\left(E_{0}^{\bullet}, d_{c}\right)$ by the usual de Rham complex $\left(\Omega^{\bullet}, d\right)$ in $\mathbb{R}^{2 n+1}$, then the $\mathbb{H}$-Poincare ${ }_{p, q}$ inequality holds on Euclidean balls for $h=1$ and $n>p \geqslant 1$. If $h>1$, then the $\mathbb{H}$-Poincaré $_{p, q}$ inequality for $2 n+1>p>1$ and $\frac{1}{p}-\frac{1}{q}=$ $\frac{1}{2 n+1}$ is proved by Iwaniec \& Lutoborski (see [33, Corollary 4.2]).

We give below a statement that deals with $\mathbb{H}-$ Sobolev inequality.

Definition 5.5. Take $\lambda>1$ and set $B=B(e, 1)$ and $B^{\prime}=B(e, \lambda)$. If $1 \leqslant h \leqslant 2 n, 1 \leqslant p \leqslant$ $q<\infty$ and $q \geqslant p$, we say that the interior $\mathbb{H}-\operatorname{Sobolev}_{p, q}(h)$ inequality holds if there exists a constant $C$ such that for every compactly supported smooth $d_{c}$-exact differential $h$-form $\omega$ in $L^{p}\left(B ; E_{0}^{h}\right)$, there exists a smooth compactly supported differential $(h-1)$-form $\phi$ in $L^{q}\left(B^{\prime}, E_{0}^{h-1}\right)$ such that $d_{c} \phi=\omega$ in $B^{\prime}$ and

$$
\|\phi\|_{L^{q}\left(B^{\prime}, E_{0}^{h-1}\right)} \leqslant C\|\omega\|_{L^{p}\left(B, E_{0}^{h}\right)} .
$$

Here we have extended $\omega$ by 0 to all of $B^{\prime}$.

Remark 5.6. If $h=1$ and $Q>p \geqslant 1$, then ( $\left.\mathbb{H}-\operatorname{Sobolev}_{p, q}(1)\right)$ is nothing but the usual Sobolev inequality with $\frac{1}{p}-\frac{1}{q}=\frac{1}{Q}$.

In [33], starting from Cartan's homotopy formula, the authors proved that if $D \subset \mathbb{R}^{N}$ is a convex set, $1<p<\infty, 1<h<N$, then there exists a bounded linear map

$$
K_{\mathrm{Euc}, h}: L^{p}\left(D, \bigwedge^{h}\right) \rightarrow W^{1, p}\left(D, \bigwedge^{h-1}\right)
$$

which is a homotopy operator, i.e.,

$$
\omega=d K_{\mathrm{Euc}, h} \omega+K_{\mathrm{Euc}, h+1} d \omega \quad \text { for all } \omega \in C^{\infty}\left(D, \bigwedge^{h}\right)
$$


(see [33, Proposition 4.1 and Lemma 4.2]). More precisely, $K_{\text {Euc }}$ has the form

$$
K_{\mathrm{Euc}, h} \omega(x)=\int_{D} \psi(y) K_{y} \omega(x) d y,
$$

where $\psi \in \mathcal{D}(D), \int_{D} \psi(y) d y=1$, and

$$
\left\langle K_{y} \omega(x) \mid \xi_{1} \wedge \cdots \wedge \xi_{h-1}\right\rangle:=\int_{0}^{1} t^{h-1}\left\langle\omega(t x+(1-t) y) \mid(x-y) \wedge \xi_{1} \wedge \cdots \wedge \xi_{h-1}\right\rangle .
$$

Starting from [33], in [42, Theorem 4.1], the authors define a compact homotopy operator $J_{\text {Euc, } h}$ in Lipschitz star-shaped domains in the Euclidean space $\mathbb{R}^{N}$, providing an explicit representation formula for $J_{\mathrm{Euc}, h}$, together with continuity properties among Sobolev spaces. More precisely, if $D \subset \mathbb{R}^{N}$ is a star-shaped Lipschitz domain and $1<h<N$, then there exists

$$
J_{\mathrm{Euc}, h}: L^{p}\left(D, \bigwedge^{h}\right) \rightarrow W_{0}^{1, p}\left(D, \bigwedge^{h-1}\right)
$$

such that

$$
\omega=d J_{\text {Euc }, h} \omega+J_{\text {Euc }, h+1} d \omega \text { for all } \omega \in \mathcal{D}\left(D, \wedge^{h}\right) .
$$

Furthermore, $J_{\mathrm{Euc}, h}$ maps smooth compactly supported forms to smooth compactly supported forms.

Take now $D=B(e, 1)=: B$ and $N=2 n+1$. If $\omega \in C^{\infty}\left(B, E_{0}^{h}\right)$, then we set

$$
K=\Pi_{E_{0}} \circ \Pi_{E} \circ K_{\mathrm{Euc}} \circ \Pi_{E}
$$

(for the sake of simplicity, from now on we drop the index $k$ - the degree of the form writing, e.g., $K_{\text {Euc }}$ instead of $\left.K_{\text {Euc, } h k}\right)$.

Analogously, we can define

$$
J=\Pi_{E_{0}} \circ \Pi_{E} \circ J_{\mathrm{Euc}} \circ \Pi_{E} .
$$

Then $K$ and $J$ invert Rumin's differential $d_{c}$ on closed forms of the same degree. More precisely, we have the following lemma.

Lemma 5.7. If $\omega$ is a smooth $d_{c}$-exact differential form, then

$$
\omega=d_{c} K \omega \quad \text { if } 1 \leqslant h \leqslant 2 n+1 \quad \text { and } \quad \omega=d_{c} J \omega \quad \text { if } 1 \leqslant h \leqslant 2 n+1 .
$$

In addition, if $\omega$ is compactly supported in $B$, then $J \omega$ is still compactly supported in $B$.

Proof. We prove for instance the identity for $d_{c} K \omega$. If $d_{c} \omega=0$, then $d\left(\Pi_{E} \omega\right)=0$, and hence

$$
\Pi_{E} \omega=d K_{\mathrm{Euc}}\left(\Pi_{E} \omega\right),
$$

by (33). We recall now that by Theorem 2.7(ii) and (iv), $d \Pi_{E}=\Pi_{E} d$ and both $\Pi_{E} \Pi_{E_{0}}$ $\Pi_{E}=\Pi_{E}$ and $\Pi_{E_{0}} \Pi_{E} \Pi_{E_{0}}=\Pi_{E_{0}}$. Thus, by (36),

$$
\begin{aligned}
d_{c} K \omega & =\Pi_{E_{0}} d \Pi_{E} \Pi_{E_{0}} \Pi_{E} K_{\mathrm{Euc}} \Pi_{E} \omega=\Pi_{E_{0}} d \Pi_{E} K_{\mathrm{Euc}} \Pi_{E} \omega \\
& =\Pi_{E_{0}} \Pi_{E} d K_{\mathrm{Euc}} \Pi_{E} \omega=\Pi_{E_{0}} \Pi_{E} \Pi_{E} \omega=\Pi_{E_{0}} \Pi_{E} \omega \\
& =\Pi_{E_{0}} \Pi_{E} \Pi_{E_{0}} \omega=\Pi_{E_{0}} \omega=\omega
\end{aligned}
$$

since $\omega \in E_{0}^{\bullet}$. Finally, if $\operatorname{supp} \omega \subset B$, then supp $J \omega \subset B$ since both $\Pi_{E}$ and $\Pi_{E_{0}}$ preserve the support. 
Lemma 5.8. Put $B=B(e, 1)$. Then

(i) if $1<p<\infty$ and $h=1, \ldots, 2 n+1$, then $K: W^{1, p}\left(B, E_{0}^{h}\right) \rightarrow L^{p}\left(B, E_{0}^{h-1}\right)$ is bounded;

(ii) if $1<p<\infty$ and $n+1<h \leqslant 2 n+1$, then $K: L^{p}\left(B, E_{0}^{h}\right) \rightarrow L^{p}\left(B, E_{0}^{h-1}\right)$ is compact;

(iii) if $1<p<\infty$ and $h=n+1$, then $K: L^{p}\left(B, E_{0}^{n+1}\right) \rightarrow L^{p}\left(B, E_{0}^{n}\right)$ is bounded.

Analogous assertions hold for $1 \leqslant h \leqslant 2 n+1$ when we replace $K$ by $J$. In addition, $\operatorname{supp} J \omega \subset B$.

Proof. By its very definition, $\Pi_{E}: W^{1, p}\left(B, E_{0}^{h}\right) \rightarrow L^{p}\left(B, E_{0}^{h}\right)$ is bounded. By $(32), K_{\text {Euc }}$ is continuous from $L^{p}\left(B, E_{0}^{h}\right)$ to $W^{1, p}\left(B, E_{0}^{h-1}\right)$. Then we can conclude the proof of (i), taking again into account that $\Pi_{E}$ is a differential operator of order $\leqslant 1$ in the horizontal derivatives.

To prove (ii), it is enough to recall that $K=\Pi_{E_{0}} K_{\text {Euc }}$ on forms of degree $h>n$, together with [33, Remark 4.1].

As for (iii), the statement can be proved similarly to (i), noting that $K=\Pi_{E_{0}} \Pi_{E} K_{\text {Euc }}$ on forms of degree $n+1$.

Finally, supp $J \omega \subset B$ since both $\Pi_{E}$ and $\Pi_{E_{0}}$ preserve the support.

The operators $K$ and $J$ provide a local homotopy in the Rumin complex, but fail to yield the Sobolev and Poincaré inequalities we are looking for since, because of the presence of the projection operator $\Pi_{E}$ (which on forms of low degree is a first-order differential operator), they lose regularity as is stated in Lemma 5.8 (ii). In order to build 'good' local homotopy operators with the desired gain of regularity, we have to combine them with homotopy operators which, though not local, in fact provide the 'good' gain of regularity.

Proposition 5.9. If $\alpha \in \mathcal{D}\left(\mathbb{H}^{n}, E_{0}^{h}\right)$ for $p>1$ and $h=1, \ldots, 2 n$, then the following homotopy formulas hold: there exist operators $K_{1}, \tilde{K}_{1}$ and $K_{2}, \tilde{K}_{1}$ acting on $\mathcal{D}\left(\mathbb{H}^{n}, E_{0}^{\bullet}\right)$ such that

- if $h \neq n, n+1$, then $\alpha=d_{c} K_{1} \alpha+\tilde{K}_{1} d_{c} \alpha$, where $K_{1}$ and $\tilde{K}_{1}$ are associated with kernels $k_{1}, \tilde{k}_{1}$ of type 1 ;

- if $h=n$, then $\alpha=d_{c} K_{1} \alpha+\tilde{K}_{2} d_{c} \alpha$, where $K_{1}$ and $\tilde{K}_{2}$ are associated with kernels $k_{1}, \tilde{k}_{2}$ of types 1 and 2 , respectively;

- if $h=n+1$, then $\alpha=d_{c} K_{2} \alpha+\tilde{K}_{1} d_{c} \alpha$, where $K_{2}$ and $\tilde{K}_{1}$ are associated with kernels $k_{2}, \tilde{k}_{1}$ of types 2 and 1 , respectively.

Proof. Suppose $h \neq n-1, n, n+1$. By Lemma 3.15, we have

$$
\begin{aligned}
\alpha & =\Delta_{\mathbb{H}, h} \Delta_{\mathbb{H}, h}^{-1} \alpha=d_{c}\left(\delta_{c} \Delta_{\mathbb{H}, h}^{-1}\right) \alpha+\delta_{c}\left(d_{c} \Delta_{\mathbb{H}, h}^{-1}\right) \alpha \\
& =d_{c}\left(\delta_{c} \Delta_{\mathbb{H}, h}^{-1}\right) \alpha+\left(\delta_{c} \Delta_{\mathbb{H}, h+1}^{-1}\right) d_{c} \alpha
\end{aligned}
$$

where $\delta_{c} \Delta_{\mathbb{H}, h}^{-1}$ and $\delta_{c} \Delta_{\mathbb{H}, h+1}^{-1}$ are associated with a kernel of type 1 (by Proposition 3.3 and Theorem 3.12). 
Analogously, if $h=n-1$,

$$
\begin{aligned}
\alpha & =\Delta_{\mathbb{H}, n-1} \Delta_{\mathbb{H}, n-1}^{-1} \alpha=d_{c}\left(\delta_{c} \Delta_{\mathbb{H}, n-1}^{-1}\right) \alpha+\delta_{c}\left(d_{c} \Delta_{\mathbb{H}, n-1}^{-1}\right) \alpha \\
& =d_{c}\left(\delta_{c} \Delta_{\mathbb{H}, n-1}^{-1}\right) \alpha+\left(\delta_{c} d_{c} \delta_{c} \Delta_{\mathbb{H}, n}^{-1}\right) d_{c} \alpha .
\end{aligned}
$$

Again $\delta_{c} \Delta_{\mathbb{H}, n-1}^{-1}$ and $\delta_{c} d_{c} \delta_{c} \Delta_{\mathbb{H}, n}^{-1}$ are associated with kernels of type 1 .

Take now $h=n$. Then

$$
\begin{aligned}
\alpha & =\Delta_{\mathbb{H}, n} \Delta_{\mathbb{H}, n}^{-1} \alpha=\left(d_{c} \delta_{c}\right)^{2} \Delta_{\mathbb{H}, n}^{-1} \alpha+\delta_{c}\left(d_{c} \Delta_{\mathbb{H}, n}^{-1}\right) \alpha \\
& =d_{c}\left(\delta_{c} d_{c} \delta_{c} \Delta_{\mathbb{H}, n}^{-1}\right) \alpha+\delta_{c} \Delta_{\mathbb{H}, n+1}^{-1} d_{c} \alpha,
\end{aligned}
$$

where $\delta_{c} d_{c} \delta_{c} \Delta_{\mathbb{H}, n}^{-1}$ and $\delta_{c} \Delta_{\mathbb{H}, n+1}^{-1}$ are associated with kernels of types 1 and 2 , respectively.

Finally, take $h=n+1$. Then

$$
\begin{aligned}
\alpha & =\Delta_{\mathbb{H}, n+1} \Delta_{\mathbb{H}, n+1}^{-1} \alpha=d_{c} \delta_{c} \Delta_{\mathbb{H}, n+1}^{-1} \alpha+\left(\delta_{c} d_{c}\right)^{2} \Delta_{\mathbb{H}, n+1}^{-1} \alpha \\
& =d_{c} \delta_{c} \Delta_{\mathbb{H}, n+1}^{-1} \alpha+\delta_{c} \Delta_{\mathbb{H}, n+2}^{-1} d_{c} \alpha,
\end{aligned}
$$

where $\delta_{c} \Delta_{\mathbb{H}, n+1}^{-1}$ and $\delta_{c} \Delta_{\mathbb{H}, n+2}^{-1}$ are associated with kernels of types 2 and 1 , respectively.

The $L^{p}-L^{q}$ continuity properties of convolution operators associated with Folland's kernels yield the following global $\mathbb{H}$-Poincaré ${ }_{p, q}(h)$ inequality in $\mathbb{H}^{n}$ (the global $\mathbb{H}-$ Sobolev $_{p, q}(h)$ is obtained in Corollary 5.21).

Corollary 5.10. Take $1 \leqslant h \leqslant 2 n+1$. Suppose $1<p<Q$ if $h \neq n+1$ and $1<p<Q / 2$ if $h=n+1$. Let $q \geqslant p$ defined by

$$
\frac{1}{p}-\frac{1}{q}:= \begin{cases}\frac{1}{Q} & \text { if } h \neq n+1, \\ \frac{2}{Q} & \text { if } h=n+1 .\end{cases}
$$

Then for any exact form $\alpha \in \mathcal{D}\left(\mathbb{H}^{n}, E_{0}^{h}\right)$, there exists $\phi \in L^{q}\left(\mathbb{H}^{n}, E_{0}^{h-1}\right)$ such that $d_{c} \phi=\alpha$ and

$$
\|\phi\|_{L^{q}\left(\mathbb{H}^{n}, E_{0}^{h-1}\right)} \leqslant C\|\alpha\|_{L^{p}\left(\mathbb{H}^{n}, E_{0}^{h-1}\right)}
$$

(i.e., the global $\mathbb{H}$-Poincaré $p, q(h)$ inequality holds for $1 \leqslant h \leqslant 2 n+1$ ).

Example 5.11. Suppose for instance $n=1$. In this case $Q=4$ and, keeping in mind Example 2.8, $\alpha=\alpha_{1} d x+\alpha_{2} d y \in E_{0}^{1}$, then Corollary 5.10 yields that there exists a function $\phi$ such that

$$
X \phi=\alpha_{1}, \quad Y \phi=\alpha_{2} .
$$

Moreover, if $\alpha=\alpha_{13} d x \wedge \theta+\alpha_{23} d y \wedge \theta \in E_{0}^{2}$, then there exists $\phi=\phi_{1} d x+\phi_{2} d y \in E_{0}^{1}$ such that

$$
X^{2} \phi_{2}-2 X Y \phi_{1}+Y X \phi_{1}=\alpha_{13} \quad \text { and } \quad 2 Y X \phi_{2}-Y^{2} \phi_{1}-X Y \phi_{2}=\alpha_{23} .
$$


Theorem 5.12. Let $B=B(e, 1)$ and $B^{\prime}=B(e, \lambda), \lambda>1$, be concentric balls of $\mathbb{H}^{n}$. If $1 \leqslant h \leqslant 2 n+1$, there exist operators $T$ and $\tilde{T}$ from $C^{\infty}\left(B^{\prime}, E_{0}^{h}\right)$ to $C^{\infty}\left(B, E_{0}^{h-1}\right)$ and $S$ from $C^{\infty}\left(B^{\prime}, E_{0}^{h}\right)$ to $C^{\infty}\left(B, E_{0}^{h}\right)$ satisfying

$$
d_{c} T+\tilde{T} d_{c}+S=I \quad \text { on } B .
$$

Proof. Suppose first $h \neq n, n+1$. We consider a cut-off function $\psi_{R}$ supported in an $R$-neighborhood of the origin such that $\psi_{R} \equiv 1$ near the origin. With the notations of Proposition 5.9, we can write

$$
k_{1}=k_{1} \psi_{R}+\left(1-\psi_{R}\right) k_{1} \quad \text { and } \quad \tilde{k}_{1}=\tilde{k}_{1} \psi_{R}+\left(1-\psi_{R}\right) \tilde{k}_{1},
$$

where

$$
k_{1}=:\left(k_{1}\right)_{\ell, \lambda} \text { and } \tilde{k}_{1}=:\left(\tilde{k}_{1}\right)_{\ell, \lambda}
$$

are the matrix-valued kernels associated with the operators $\delta_{c} \Delta_{\mathbb{H}, h}$ and $\delta_{c} \Delta_{\mathbb{H}, h+1}$, respectively, as shown in the proof of Proposition 5.9.

Let us denote by $K_{1, R}, \tilde{K}_{1, R}$ the convolution operators associated with $\psi_{R} k_{1}, \psi_{R} \tilde{k}_{1}$, respectively. Let us fix two balls $B_{0}, B_{1}$ with

$$
B \Subset B_{0} \Subset B_{1} \Subset B^{\prime},
$$

and a cut-off function $\chi \in \mathcal{D}\left(B_{1}\right), \chi \equiv 1$ on $B_{0}$. If $\alpha \in C^{\infty}\left(B^{\prime}, E_{0}^{\bullet}\right)$, we set $\alpha_{0}=\chi \alpha$, continued by zero outside $B_{1}$.

We have

$$
\alpha_{0}=d_{c} K_{1, R} \alpha_{0}+\tilde{K}_{1, R} d_{c} \alpha_{0}+S_{0} \alpha_{0}
$$

where $S_{0}$ is

$$
S_{0} \alpha_{0}:=d_{c}\left(\alpha_{0} *\left(1-\psi_{R}\right) k_{1}\right)+d_{c} \alpha_{0} *\left(1-\psi_{R}\right) \tilde{k}_{1}
$$

We set

$$
T \alpha:=K_{1, R} \alpha_{0}, \quad \tilde{T} d_{c} \alpha:=\tilde{K}_{1, R} d_{c} \alpha_{0}, \quad S \alpha:=S_{0} \alpha_{0} .
$$

We note that, provided $R>0$ is small enough, the definition of $T$ and $\tilde{T}$ does not depend on the continuation of $\alpha$ outside $B_{0}$. By (44), we have

$$
\alpha=d_{c} T \alpha+\tilde{T} d_{c} \alpha+S \alpha \quad \text { in } B
$$

If $h=n$, we can carry out the same construction, replacing $\tilde{k}_{1}$ by $\tilde{k}_{2}$ (keep in mind that $\tilde{k}_{2}$ is a kernel of type 2, again by Proposition 5.9). Analogously, if $h=n+1$, we can carry out the same construction, replacing $k_{1}$ by $k_{2}$ (again a kernel of type 2 ).

Later on, we need the following remark.

Remark 5.13. By construction, if $\operatorname{supp} \alpha \subset B$, then $\operatorname{supp} T \alpha$ is contained in an $R$-neighborhood of $B$ and then is contained in $B_{0}$ provided $R<d\left(B, \partial B_{0}\right)$.

The homotopies $T$ and $\tilde{T}$ provide the desired 'gain of regularity' as stated in the following theorem. 
Theorem 5.14. Let $B=B(e, 1)$ and $B^{\prime}=B(e, \lambda), \lambda>1$, be concentric balls of $\mathbb{H}^{n}$, and let $1 \leqslant h \leqslant 2 n+1$. If $T, \tilde{T}$ are as in Theorem 5.12, then we have the following:

(i) $\tilde{T}: W^{-1, p}\left(B^{\prime}, E_{0}^{h+1}\right) \rightarrow L^{p}\left(B, E_{0}^{h}\right)$ if $h \neq n$, and $\tilde{T}: W^{-2, p}\left(B, E_{0}^{n+1}\right) \rightarrow L^{p}\left(B, E_{0}^{n}\right)$.

(ii) $T: L^{p}\left(B^{\prime}, E_{0}^{h}\right) \rightarrow W^{1, p}\left(B, E_{0}^{h-1}\right), \quad h \neq n+1$, whereas $T: L^{p}\left(B^{\prime}, E_{0}^{n+1}\right) \rightarrow W^{2, p}$ $\left(B, E_{0}^{n}\right)$,

so that (40) still holds in $L^{p}\left(B, E_{0}^{\bullet}\right)$.

In addition, for every $(h, p, q)$ satisfying inequalities

$$
1<p \leqslant q<\infty, \quad \frac{1}{p}-\frac{1}{q} \leqslant \begin{cases}\frac{1}{Q} & \text { if } h \neq n+1 \\ \frac{2}{Q} & \text { if } h=n+1\end{cases}
$$

we have

(iii) $T: L^{p}\left(B^{\prime}, E_{0}^{h}\right) \rightarrow L^{q}\left(B, E_{0}^{h-1}\right)$.

Proof. Let us prove (i). Suppose $h \neq n$, and take $\beta \in W^{-1, p}\left(B^{\prime}, E_{0}^{h+1}\right)$. As in the proof of the previous theorem, let $\psi_{R}$ be a cut-off function supported in an $R$-neighborhood of the origin such that $\psi_{R} \equiv 1$ near the origin. Thus, again with the notations of the proof of the previous theorem (see, in particular, (46) and (42)), the operator $\tilde{K}_{1, R}$ is associated with a matrix-valued kernel $\psi_{R}\left(\tilde{k}_{1}\right)_{\ell, \lambda}$ and $\beta$ is identified with a vector-valued distribution $\left(\beta_{1}, \ldots, \beta_{N_{h}}\right)$, with $\beta_{j}=\sum_{i} W_{i} f_{i}^{j}$ as in Remark 4.7, with

$$
\sum_{j} \sum_{i}\left\|f_{i}^{j}\right\|_{L^{p}\left(B^{\prime}\right)} \leqslant C\|\beta\|_{W^{-1, p}\left(B^{\prime}, E_{0}^{h+1}\right)} .
$$

As in the proof of the previous theorem, let us fix two balls $B_{0}, B_{1}$ with $B \Subset B_{0} \Subset B_{1} \Subset B^{\prime}$. If $\chi \in \mathcal{D}\left(B_{1}\right)$ is a cut-off function such that $\chi \equiv 1$ on $B_{0}$, we set $\beta_{0}=\chi \beta$. Thus $\left(\beta_{0}\right)_{j}$, the $j$ th component of $\beta_{0}$, has the form

$$
\left(\beta_{0}\right)_{j}=\sum_{i} W_{i}\left(\chi f_{i}^{j}\right)-\sum_{i}\left(W_{i} \chi\right) f_{i}^{j} .
$$

Keeping in mind Remark 4.7, in order to estimate the norm of $\tilde{T} \beta$ in $L^{p}\left(B, E_{0}^{h}\right)$, we estimate $\langle\tilde{T} \beta \mid \phi\rangle$, where

$$
\phi=\sum_{j} \phi_{j} \xi_{j}^{h} \in \mathcal{D}\left(B, E_{0}^{h}\right), \quad \text { with }\|\phi\|_{L^{p^{\prime}}\left(B^{\prime}, E_{0}^{h}\right)} \leqslant 1 .
$$

By $(29),\langle\tilde{T} \beta \mid \phi\rangle$ is a sum of terms of the form

$$
\int_{B}\left(\psi_{R} \kappa * f_{0}\right)(x) W_{i} \phi(x) d x=\left\langle\psi_{R} \kappa * W_{i} f_{0} \mid \phi\right\rangle
$$

(where, as above, $f_{0}=\chi f$ ) or of the form

$$
\int_{B}\left(\psi_{R} \kappa *\left(W_{i} \chi\right) f\right)(x) \phi(x) d x
$$


where $\kappa$ denotes one of the kernels $\left(\tilde{k}_{1}\right)_{\ell, \lambda}$ of type 1 associated with $\tilde{k}_{1}$ (see $(41)$ in the proof of the previous theorem), $f$ is one of the $f_{i}^{j}$ 's and $\phi$ is one of the $\phi_{j}$ 's.

As for (48), by (23),

$$
\begin{aligned}
\left\langle\psi_{R} \kappa * W_{i} f_{0} \mid \phi\right\rangle & =\left\langle{ }^{\mathrm{v}} W^{I}{ }^{\mathrm{v}}\left[\psi_{R} \kappa\right] * f_{0} \mid \phi\right\rangle \\
& =\left\langle\psi_{R}{ }^{\mathrm{v}} W^{I}{ }^{I} \kappa * f_{0} \mid \phi\right\rangle-\left\langle\left({ }^{\mathrm{v}} W^{I \mathrm{v}} \psi_{R}\right) \kappa * f_{0} \mid \phi\right\rangle .
\end{aligned}
$$

We note now that ${ }^{\mathrm{v}} W^{I}{ }^{\mathrm{v}} \boldsymbol{\kappa}$ is a kernel of type 0 . Therefore, by Lemma 3.5 (keep in mind that $f_{0}$ and $\phi$ are real functions),

$$
\begin{aligned}
\left\langle\psi_{R}{ }^{\mathrm{v}} W^{I}{ }^{\mathrm{v}} \kappa * f_{0} \mid \phi\right\rangle & \leqslant\left\|\psi_{R}{ }^{\mathrm{v}} W^{I}{ }^{\mathrm{v}} \kappa * f_{0}\right\|_{L^{p}(B)}\|\phi\|_{L^{p^{\prime}}(B)} \\
& \leqslant\left\|\psi_{R}{ }^{\mathrm{v}} W^{I}{ }{ }_{\kappa} * f_{0}\right\|_{L^{p}(B)} \leqslant C\left\|f_{0}\right\|_{L^{p}\left(B^{\prime}\right)} \\
& \leqslant C\|\beta\|_{W^{-1, p}\left(B^{\prime}, E_{0}^{h+1}\right)} .
\end{aligned}
$$

The term in (49) can be handled in the same way, taking into account Remark (3.6). Eventually, combining (48) and (49), we obtain that

$$
\|\tilde{T} \beta\|_{L^{p}\left(B, E_{0}^{h}\right)} \leqslant C\|\beta\|_{W^{-1, p}\left(B^{\prime}, E_{0}^{h+1}\right)} .
$$

The assertion for $h=n$ can be proved in the same way, taking into account that $\tilde{T}$ is built from a kernel of type 2 and that the norm in the space $W^{-2, p}\left(B, E_{0}^{n+1}\right)$ is expressed by duality in terms of second-order horizontal derivatives of test functions (see Remark 4.5).

Let us prove now (ii). Suppose $h \neq n+1$ and take $\alpha=\sum_{j} \alpha_{j} \xi_{j}^{h} \in \mathcal{D}\left(B^{\prime}, E_{0}^{h}\right)$. Arguing as above, in order to estimate $\|T \alpha\|_{W^{1, p}\left(B, E_{0}^{h-1}\right)}$, we have to consider terms of the form

$$
W_{\ell}\left(\psi_{R} \kappa *\left(\chi \alpha_{j}\right)\right)=\psi_{R} \kappa *\left(W_{\ell}\left(\chi \alpha_{j}\right)\right)
$$

(when we want to estimate the $L^{p}$-norm of the horizontal derivatives of $T \alpha$ ) or of the form

$$
\psi_{R} \kappa *\left(\chi \alpha_{j}\right)
$$

(when we want to estimate the $L^{p}$-norm of $T \alpha$ ). Both (50) and (51) can be handled as in case (i) (no need here of the duality argument).

We point out that (51) yields an $L^{p}-L^{q}$ estimate (since, unlike (50), it involves only kernels of type 1) and then assertion (iii) follows.

The operator $S$ is the required local smoothing operator. More precisely, we have the following theorem.

Theorem 5.15. Let $B=B(e, 1)$ and $B^{\prime}=B(e, \lambda), \lambda>1$, be concentric balls of $\mathbb{H}^{n}$, and let $1 \leqslant h \leqslant 2 n+1$. Then the operator $S$ defined in (46) is a smoothing operator. In particular, for any $m, s \in \mathbb{Z}, m<s, S$ is bounded from $W^{m, p}\left(B^{\prime}, E_{0}^{h}\right)$ to $W^{s, q}\left(B, E_{0}^{h}\right)$ for any $p, q \in$ $(1, \infty)$ and maps $W^{m, p}\left(B^{\prime}, E_{0}^{h}\right)$ into $C^{\infty}\left(B, E_{0}^{h}\right)$.

Proof. Since $B$ is bounded, we can assume $q>p$. First, take $m=0$. Again, let us fix two balls $B_{0}, B_{1}$ with $B \Subset B_{0} \Subset B_{1} \Subset B^{\prime}$. If $\chi \in \mathcal{D}\left(B_{1}\right)$ is a cut-off function such that $\chi \equiv 1$ on $B_{0}$, we set $\alpha_{0}=\chi \alpha$. Keeping the notations of the proof of Theorem 5.12 , it is easy to 
check that $S \alpha$ can be written as (see (45))

$$
\left.S \alpha=S_{0} \alpha_{0}:=\alpha_{0} * d_{c}\left(1-\psi_{R}\right) k_{1}\right) \pm \alpha_{0} *^{\mathrm{v}} d_{c}{ }^{\mathrm{v}}\left(1-\psi_{R}\right) \tilde{k}_{1} .
$$

Thus, if $\alpha=\sum_{j} \alpha_{j} \xi_{j}^{h}$, then each entry of $S \alpha$ is a sum of terms of the form

$$
\left(\chi \alpha_{j}\right) * \kappa,
$$

where $\kappa$ is a smooth kernel. Thus we are led to estimate the $L^{q}$-norms in $B$ of a sum of terms of the form

$$
\left(\chi \alpha_{j}\right) * W^{J} \kappa=\left(\chi \alpha_{j}\right) * \mathbf{1}_{2 B^{\prime}} W^{J} \kappa \quad \text { with }|J|=s,
$$

and the assertion follows by the classical Hausdorff-Young inequality (see [20, Proposition 1.10]) since the kernel $\mathbf{1}_{2 B^{\prime}} W^{J} \kappa$ belongs to all $L^{r}, r \geqslant 1$. Therefore $S$ is bounded from $L^{p}\left(B^{\prime}, E_{0}^{h}\right)$ to $W^{s, q}\left(B, E_{0}^{h}\right)$. Clearly, this yields the continuity of $S$ from $W^{m, p}\left(B^{\prime}, E_{0}^{h}\right)$ to $W^{s, q}\left(B, E_{0}^{h}\right)$ for $m \geqslant 0$.

The proof in the case $m<0$ can be carried out by a duality argument akin to the one we used in the proof of Theorem 5.14.

Remark 5.16. Apparently, in the previous theorem, two different homotopy operators $T$ and $\tilde{T}$ appear. In fact, they coincide when acting on the form of the same degree.

More precisely, in Proposition 5.9, the homotopy formulas involve four operators $K_{1}, \tilde{K}_{1}, K_{2}, \tilde{K}_{2}$, where the notation is meant to distinguish operators acting on $d_{c} \alpha$ (the operators with tilde) from those on which the differential acts (the operators without tilde), whereas the lower index 1 or 2 denotes the type of the associated kernels. Alternatively, a different notation could be used: if $\alpha \in \mathcal{D}\left(\mathbb{H}^{n}, E_{0}^{h}\right)$, we can write

$$
\alpha=d_{c} K_{h}+\tilde{K}_{h+1} d_{c} \alpha,
$$

where the tilde has the same previous meaning, whereas the lower index refers now to the degree of the forms on which the operator acts.

It is important to note that

$$
K_{h+1}=\tilde{K}_{h+1}, \quad h=1, \ldots, 2 n .
$$

Indeed, take $h<n-1$. Then $\tilde{K}_{h+1}=\delta_{c} \Delta_{\mathbb{H}, h+1}^{-1}$ (as it appears in the homotopy formula at the degree $h$ ), which equals $K_{h+1}$ (as it appears in the homotopy formula at the degree $h+1 \leqslant n-1$ ). Take now $h=n-1$. Then $\tilde{K}_{n}=\delta_{c} d_{c} \delta_{c} \Delta_{\mathbb{H}, n}^{-1}$ (as it appears in the homotopy formula at the degree $n$ ), which equals $K_{n}$ (as it appears in the homotopy formula at the degree $n$ ). If $h=n$, then $\tilde{K}_{n+1}=\delta_{c} \Delta_{\mathbb{H}, n+1}^{-1}$ (as it appears in the homotopy formula at the degree $n$ ), which equals $K_{n+1}$ (as it appears in the homotopy formula at the degree $n+1)$. Finally, if $h>n$, then $\tilde{K}_{h+1}=\delta_{c} \Delta_{\mathbb{H}, h+1}^{-1}$ (as it appears in the homotopy formula at the degree $h$ ), which equals $K_{h+1}$ (as it appears in the homotopy formula at the degree $h+1$ ).

Once this point is established, from now on, we shall write

$$
K:=K_{h}=\tilde{K}_{h}
$$

without ambiguity. 
Therefore $T=\tilde{T}$, and the homotopy formula (40) reads as

$$
d_{c} T+T d_{c}+S=I \quad \text { on } B
$$

It is worth pointing out the following fact.

Remark 5.17. As above, take $B:=B(e, 1), B^{\prime}:=B(e, \lambda)$ with $\lambda>1$, and as in formula (43), let us fix two balls $B_{0}, B_{1}$ with

$$
B \Subset B_{0} \Subset B_{1} \Subset B^{\prime},
$$

and a cut-off function $\chi \in \mathcal{D}\left(B_{1}\right), \chi \equiv 1$ on $B_{0}$.

Take now $\alpha, \beta \in L^{p}\left(B^{\prime}, E_{0}^{\bullet}\right), \alpha \equiv \beta$ on $B_{1}$. Then, by (53), $S \alpha=S \beta$ in $B$. Indeed, if $\alpha_{0}:=\chi \alpha \equiv \beta_{0}:=\chi \beta$ in $B_{0}$, then $K_{1, R} \alpha_{0} \equiv K_{1, R} \beta_{0}$ and $\tilde{K}_{1, R} d_{c} \alpha_{0} \equiv \tilde{K}_{1, R} d_{c} \tilde{\beta}_{0}$ in $B$. In other words, $\left(d_{c} T+T d_{c}\right) \alpha=\left(d_{c} T+T d_{c}\right) \beta$ in $B$.

The following commutation lemma will be helpful in the sequel.

Lemma 5.18. As above, take $B:=B(e, 1), B^{\prime}:=B(e, \lambda)$ with $\lambda>1$, and as in formula (43), let us fix two balls $B_{0}, B_{1}$ with

$$
B \Subset B_{0} \Subset B_{1} \Subset B^{\prime},
$$

and a cut-off function $\chi \in \mathcal{D}\left(B_{1}\right), \chi \equiv 1$ on $B_{0}$.

We have

$$
S d_{c} \alpha=d_{c} S \alpha \quad \text { for all } \alpha \in L^{p}\left(\mathbb{H}^{n}, E_{0}^{h}\right),
$$

$1 \leqslant h \leqslant 2 n+1$.

Proof. By (53), if $\alpha \in \mathcal{D}\left(B^{\prime}, E_{0}^{h}\right)$, then $S d_{c} \alpha=d_{c} S \alpha$. The case $\alpha \in L^{p}\left(B^{\prime}, E_{0}^{h}\right)$ requires more technicalities.

Indeed, take $\alpha \in L^{p}\left(B^{\prime}, E_{0}^{h}\right)$, and let $\chi_{1}$ be a cut-off function supported in $B^{\prime}, \chi_{1} \equiv 1$ on $B_{1}$. By convolution with the usual Friedrichs mollifiers (see Definition 3.1), we can find a sequence $\left(\alpha_{k}\right)_{k \in \mathbb{N}}$ in $\mathcal{D}\left(B^{\prime}, E_{0}^{h}\right)$ converging to $\chi_{1} \alpha$ in $L^{p}\left(B^{\prime}, E_{0}^{h}\right)$. By Theorem 5.14 , $S \alpha_{k} \rightarrow S\left(\chi_{1} \alpha\right)$ in $W^{2, p}\left(B, E_{0}^{h+1}\right)$, and hence $d_{c} S \alpha_{k} \rightarrow d_{c} S\left(\chi_{1} \alpha\right)$ in $L^{p}\left(B, E_{0}^{h}\right)$ as $k \rightarrow \infty$.

On the other hand, $\chi_{1} \alpha \equiv \alpha$ in $B_{1}$, and then by Remark 5.17, $S\left(\chi_{1} \alpha\right)=S \alpha$ in $B$ so that $d_{c} S \alpha_{k} \rightarrow d_{c} S \alpha$ in $L^{p}\left(B, E_{0}^{h}\right)$ as $k \rightarrow \infty$.

In addition, $d_{c} \alpha_{k} \rightarrow d_{c}\left(\chi_{1} \alpha\right)$ in $W^{-1, p}\left(B^{\prime}, E_{0}^{h}\right)$ (in $W^{-2, p}\left(B^{\prime}, E_{0}^{h}\right)$ if $h=n$ ), and hence by Theorem 5.12, $S d_{c} \alpha_{k} \rightarrow S d_{c}\left(\chi_{1} \alpha\right)$ in $B$ as $k \rightarrow \infty$. Again, $d_{c}\left(\chi_{1} \alpha\right) \equiv d_{c} \alpha$ in $B_{1}$, and then by Remark 5.17, $S d_{c} \alpha_{k} \rightarrow S d_{c} \alpha$ in $B$ as $k \rightarrow \infty$.

Finally, since $d_{c} S \alpha_{k}=S d_{c} \alpha_{k}$ for all $k \in \mathbb{N}$, we can take the limits as $k \rightarrow \infty$ and the assertion follows.

The following theorem contains one of the main results of the paper: it yields the interior Poincaré inequality and Sobolev inequality for Rumin forms in the sense of Definitions 5.1 and 5.5. 
Theorem 5.19. Take $\lambda>1$ and set $B=B(e, 1)$ and $B^{\prime}=B(e, \lambda)$. If $1 \leqslant h \leqslant 2 n+1$, as in (47), take

$$
1<p \leqslant q<\infty, \quad \frac{1}{p}-\frac{1}{q} \leqslant \begin{cases}\frac{1}{Q} & \text { if } h \neq n+1, \\ \frac{2}{Q} & \text { if } h=n+1 .\end{cases}
$$

Then we have the following:

(i) An interior $\mathbb{H}$-Poincaré ${ }_{p, q}(h)$ inequality holds with respect to the balls $B$ and $B^{\prime}$.

(ii) In addition, an interior $\mathbb{H}-\operatorname{Sobolev}_{p, q}(h)$ inequality holds for $1 \leqslant h \leqslant 2 n$.

Proof. (i) Interior $\mathbb{H}$-Poincaré ${ }_{p, q}(h)$ inequality: let $\omega \in L^{p}\left(B^{\prime}, E_{0}^{h}\right)$ be $d_{c^{-}}$-closed. By (53), we can write

$$
\omega=d_{c} T \omega+S \omega \text { in } B .
$$

By Theorem 5.15, we have $S \omega \in \mathcal{C}^{\infty}\left(B, E_{0}^{h}\right)$. Furthermore, $d_{c} S \omega=0$ since $d_{c} \omega=d_{c}^{2} T \omega+$ $d_{c} S \omega$ in $B$ and $d_{c} \omega=0$ (by assumption).

Thus we can apply (38) to $S \omega$ and we get $S \omega=d_{c} K S \omega$, where $K$ is defined in (36). In $B$, put now

$$
\phi:=(K S+T) \omega .
$$

Trivially, $d_{c} \phi=d_{c} K S \omega+d_{c} T \omega=S \omega+d_{c} T \omega=\omega$, by (56). By Theorems 5.14 and 5.15,

$$
\begin{aligned}
& \|\phi\|_{L^{q}\left(B, E_{0}^{h-1}\right)} \leqslant\|K S \omega\|_{L^{q}\left(B, E_{0}^{h-1}\right)}+\|T \omega\|_{L^{q}\left(B, E_{0}^{h-1}\right)} \\
& \quad \leqslant\|K S \omega\|_{L^{q}\left(B, E_{0}^{h-1}\right)}+C\|\omega\|_{L^{p}\left(B^{\prime}, E_{0}^{h}\right)} \\
& \quad \leqslant C\left(\|S \omega\|_{W^{1, q}\left(B, E_{0}^{h}\right)}+\|\omega\|_{L^{p}\left(B^{\prime}, E_{0}^{h}\right)}\right) \quad \text { (by Lemma 5.8) } \\
& \quad \leqslant C\|\omega\|_{L^{p}\left(B^{\prime}, E_{0}^{h}\right)} .
\end{aligned}
$$

(ii) Interior $\mathbb{H}-\operatorname{Sobolev}_{p, q}(h)$ inequality: as in formula (43), let us fix two balls $B_{0}, B_{1}$ with

$$
B \Subset B_{0} \Subset B_{1} \Subset B^{\prime},
$$

and a cut-off function $\chi \in \mathcal{D}\left(B_{1}\right), \chi \equiv 1$ on $B_{0}$.

Let $\omega \in L^{p}\left(B, E_{0}^{h}\right)$ be a compactly supported form such that $d_{c} \omega=0$. Since $\omega$ vanishes in a neighborhood of $\partial B$, without loss of generality, we can assume that it is continued by zero on $B^{\prime}$. In addition, $\omega=\chi \omega$ since $\chi \equiv 1$ on $\operatorname{supp} \omega$.

By (53), we have $\omega=d_{c} T \omega+S \omega$. On the other hand, since $\omega$ vanishes outside $B$, by its very definition (see (46)), $T \omega$ is supported in $B_{0}$ by Remark 5.13 so that also $S \omega$ is supported in $B_{0}$.

Again as above, $S \omega \in C^{\infty}\left(B, E_{0}^{h}\right)$ and $d_{c} S \omega=0$. Thus we can apply (38) to $S \omega$ and we get $S \omega=d_{c} J S \omega$, where $J$ is defined in (37) (that preserves the support). By Lemma 5.7, $J S \omega$ is supported in $B_{0} \subset B^{\prime}$. Thus, if we set $\phi:=(J S+T) \omega$, then $\phi$ is supported in $B^{\prime}$. Moreover, $d_{c} \phi=d_{c} K S \omega+d_{c} T \omega=S \omega+\omega-S \omega=\omega$.

At this point, we can repeat estimates (57) and we get eventually

$$
\|\phi\|_{L^{q}\left(B^{\prime}, E_{0}^{h-1}\right)} \leqslant C\|\omega\|_{L^{p}\left(B, E_{0}^{h}\right)} .
$$

This completes the proof of the theorem. 
If $p \in \mathbb{H}^{n}$ and $t>0$, then the map $x \rightarrow f(x):=\tau_{p} \delta_{t}(x)$ maps $B(e, \rho)$ into $B(p, t \rho)$ for $\rho>0$. Therefore, by Proposition 2.12, from the previous theorem for balls of fixed radius, we obtain the following result for general balls.

Theorem 5.20. Take $1 \leqslant h \leqslant 2 n+1$. Suppose $1<p<Q$ if $h \neq n+1$ and $1<p<Q / 2$ if $h=n+1$. Let $q \geqslant p$ such that

$$
\frac{1}{p}-\frac{1}{q} \leqslant \begin{cases}\frac{1}{Q} & \text { if } h \neq n+1 \\ \frac{2}{Q} & \text { if } h=n+1\end{cases}
$$

Then there exists a constant $C$ such that for every $d_{c}$-closed differential $h$-form $\omega$ in $L^{p}\left(B(p, \lambda r) ; E_{0}^{h}\right)$, there exists an $(h-1)$-form $\phi$ in $L^{q}\left(B(p, r), E_{0}^{h-1}\right)$ such that $d_{c} \phi=\omega$ and

$$
\|\phi\|_{L^{q}\left(B(p, r), E_{0}^{h-1}\right)} \leqslant C r^{Q / q-Q / p+1}\|\omega\|_{L^{p}\left(B(p, \lambda r), E_{0}^{h}\right)} \quad \text { if } h \neq n+1
$$

and

$$
\|\phi\|_{L^{q}\left(B(p, r), E_{0}^{n}\right)} \leqslant C r^{Q / q-Q / p+2}\|\omega\|_{L^{p}\left(B(p, \lambda r), E_{0}^{n+1}\right)} .
$$

Analogously, if $1 \leqslant h \leqslant 2 n$, there exists a constant $C$ such that for every compactly supported $d_{c}$-closed $h$-form $\omega$ in $L^{p}\left(B(p, r) ; E_{0}^{h}\right)$, there exists a compactly supported $(h-1)$-form $\phi$ in $L^{q}\left(B(p, \lambda r), E_{0}^{h-1}\right)$ such that $d_{c} \phi=\omega$ in $B(p, \lambda r)$ and

$$
\|\phi\|_{L^{q}\left(B(p, \lambda r), E_{0}^{h-1}\right)} \leqslant C\|\omega\|_{L^{p}\left(B(p, r), E_{0}^{h}\right)} .
$$

Proof. We just have to take the pull-back $f^{\#} \omega$ and then apply Theorem 5.19.

If the choice of $q$ is sharp (i.e., in (58), the equality holds), then the constant on the right-hand side of (61) is independent of the radius of the ball so that a global $\mathbb{H}-$ Sobolev $_{p, q}(h)$ inequality holds.

Therefore we get the following result.

Corollary 5.21. Take $1 \leqslant h \leqslant 2 n$. Suppose $1<p<Q$ if $h \neq n+1$ and $1<p<Q / 2$ if $h=n+1$. Let $q \geqslant p$ defined by

$$
\frac{1}{p}-\frac{1}{q}:= \begin{cases}\frac{1}{Q} & \text { if } h \neq n+1, \\ \frac{2}{Q} & \text { if } h=n+1 .\end{cases}
$$

Then the $\mathbb{H}$-Sobolev $_{p, q}(h)$ inequality holds for $1 \leqslant h \leqslant 2 n$.

In the case $\mathbb{H}^{1}$, for 1 -forms and 2 -forms for instance, the primitive $\phi$ of a compactly supported form can be written explicitly as in Example 5.11.

Remark 5.22. A scaling argument shows easily that the exponents in (59) and (60) are sharp. On the other hand, we have already discussed in $\S 1.5$ whether similar sharp results can be proved for general Carnot groups, stating ultimately that this is not possible 
(at least relying on our present arguments). Now the argument of $\S 1.5$ can be made more precise. If we look at the proofs of our inequalities, we see that at the very beginning, there is an approximate homotopy formula that in turn descends from the existence of a fundamental solution for a suitable hypoelliptic homogeneous 'artificial Laplacian'. This construction is still possible in general Carnot groups (see [8, 48]) relying on the construction of a '0-order Laplacian', but the approximate homotopy formula involves singular integral operators that fail to have the good homogeneity. This is due to the fact that in general Carnot groups, with the exception of very particular cases, the forms of a given degree in the Rumin complex have different weights (this does not happen in Euclidean spaces and in Heisenberg groups). We stress that this phenomenon appears already in step 2 groups, very akin to Heisenberg groups, like quaternionic Heisenberg groups (see [11]), which are defined by replacing the complex field $\mathbb{C}$ by the field of quaternions in the definition of $\mathbb{H}^{1}$. This generates a two-step Carnot group whose center is 3 -dimensional (while the center in $\mathbb{H}^{n}$ is 1-dimensional).

Thus the quaternionic Heisenberg group (in dimension 7) is a nilpotent Lie group with underlying manifold $\mathbb{R}_{x}^{4} \times \mathbb{R}_{t}^{3}$, where $x=\left(x_{1}, x_{2}, x_{3}, x_{4}\right)$ and $t=\left(t_{1}, t_{2}, t_{3}\right)$.

A basis for the Lie algebra of left-invariant vector fields on the group is given by

$$
\begin{gathered}
X_{1}=\frac{\partial}{\partial x_{1}}+\frac{1}{2} x_{2} \frac{\partial}{\partial t_{1}}+\frac{1}{2} x_{3} \frac{\partial}{\partial t_{2}}+\frac{1}{2} x_{4} \frac{\partial}{\partial t_{3}} ; \\
X_{2}=\frac{\partial}{\partial x_{2}}-\frac{1}{2} x_{1} \frac{\partial}{\partial t_{1}}+\frac{1}{2} x_{4} \frac{\partial}{\partial t_{2}}-\frac{1}{2} x_{3} \frac{\partial}{\partial t_{3}} ; \\
X_{3}=\frac{\partial}{\partial x_{3}}-\frac{1}{2} x_{4} \frac{\partial}{\partial t_{1}}-\frac{1}{2} x_{1} \frac{\partial}{\partial t_{2}}+\frac{1}{2} x_{2} \frac{\partial}{\partial t_{3}} ; \\
X_{4}=\frac{\partial}{\partial x_{4}}+\frac{1}{2} x_{3} \frac{\partial}{\partial t_{1}}-\frac{1}{2} x_{2} \frac{\partial}{\partial t_{2}}-\frac{1}{2} x_{1} \frac{\partial}{\partial t_{3}} ; \\
T_{k}=\frac{\partial}{\partial t_{k}} \quad \text { for } k=1,2,3 .
\end{gathered}
$$

The non-trivial commutation relations are

$$
\begin{gathered}
{\left[X_{1}, X_{2}\right]=-\left[X_{3}, X_{4}\right]=-T_{1}} \\
{\left[X_{1}, X_{3}\right]=\left[X_{2}, X_{4}\right]=-T_{2} ;} \\
{\left[X_{1}, X_{4}\right]=-\left[X_{2}, X_{3}\right]=-T_{3} .}
\end{gathered}
$$

The standard quaternionic contact forms $\tau_{1}, \tau_{2}, \tau_{3}$ are given by

$$
\begin{aligned}
\tau_{1} & =\mathrm{d} t_{1}-\frac{1}{2} x_{2} \mathrm{~d} x_{1}+\frac{1}{2} x_{1} \mathrm{~d} x_{2}-\frac{1}{2} x_{4} \mathrm{~d} x_{3}+\frac{1}{2} x_{3} \mathrm{~d} x_{4} ; \\
\tau_{2} & =\mathrm{d} t_{2}-\frac{1}{2} x_{3} \mathrm{~d} x_{1}+\frac{1}{2} x_{4} \mathrm{~d} x_{2}+\frac{1}{2} x_{1} \mathrm{~d} x_{3}-\frac{1}{2} x_{2} \mathrm{~d} x_{4} ; \\
\tau_{1} & =\mathrm{d} t_{3}-\frac{1}{2} x_{4} \mathrm{~d} x_{1}-\frac{1}{2} x_{3} \mathrm{~d} x_{2}+\frac{1}{2} x_{2} \mathrm{~d} x_{3}+\frac{1}{2} x_{1} \mathrm{~d} x_{4} .
\end{aligned}
$$

The spaces of intrinsic 1-forms and 2-forms are

$$
E_{0}^{1}=\Omega^{1,1}=\operatorname{span}\left\{d x_{1}, d x_{2}, d x_{3}, d x_{4}\right\}
$$


and

$$
E_{0}^{2}=\operatorname{span}\left\{\alpha_{2}, \alpha_{4}, \alpha_{6}\right\} \oplus \operatorname{span}\left\{\beta_{1}, \beta_{2}, \beta_{3}, \beta_{4}, \beta_{5}, \beta_{6}, \beta_{7}, \beta_{8}\right\}
$$

where

$$
\begin{array}{ll}
\alpha_{1}:=d x_{1} \wedge d x_{2}+d x_{3} \wedge d x_{4}, & \alpha_{2}:=d x_{1} \wedge d x_{2}-d x_{3} \wedge d x_{4}, \\
\alpha_{3}:=d x_{1} \wedge d x_{3}-d x_{2} \wedge d x_{4}, & \alpha_{4}:=d x_{1} \wedge d x_{3}+d x_{2} \wedge d x_{4}, \\
\alpha_{5}:=d x_{1} \wedge d x_{4}+d x_{2} \wedge d x_{3}, & \alpha_{6}:=d x_{1} \wedge d x_{4}-d x_{2} \wedge d x_{3}
\end{array}
$$

and

$$
\begin{array}{cll}
\beta_{1}:=d x_{1} \wedge \tau_{2}+d x_{4} \wedge \tau_{1}, & \beta_{2}:=d x_{2} \wedge \tau_{3}+d x_{4} \wedge \tau_{1}, & \beta_{3}:=d x_{1} \wedge \tau_{3}+d x_{2} \wedge \tau_{2}, \\
\beta_{4}:=d x_{3} \wedge \tau_{1}+d x_{2} \wedge \tau_{2}, & \beta_{5}:=d x_{1} \wedge \tau_{1}+d x_{3} \wedge \tau_{3}, & \beta_{6}:=d x_{4} \wedge \tau_{2}+d x_{3} \wedge \tau_{3}, \\
\beta_{7}:=d x_{2} \wedge \tau_{1}-d x_{4} \wedge \tau_{3}, & \beta_{8}:=-d x_{3} \wedge \tau_{2}+d x_{4} \wedge \tau_{3}, & \beta_{9}:=d x_{1} \wedge \tau_{2}-d x_{4} \wedge \tau_{1}, \\
\beta_{10}:=d x_{1} \wedge \tau_{3}-d x_{2} \wedge \tau_{2}, & \beta_{11}:=d x_{1} \wedge \tau_{1}-d x_{3} \wedge \tau_{3}, & \beta_{12}:=d x_{2} \wedge \tau_{1}+d x_{4} \wedge \tau_{3},
\end{array}
$$

respectively. It turns out that $\alpha_{2}, \alpha_{4}, \alpha_{6}$ have weight 2 , whereas $\beta_{1}, \ldots \beta_{10}$ have weight 3 .

\section{Contact manifolds and global smoothing}

Throughout this section, $(M, H, g)$ will be a sub-Riemannian contact manifold of bounded $C^{k}$-geometry as in Definition $4.9, k \geqslant 3$. We shall denote by $\left(E_{0}^{\bullet}, d_{c}\right)$ both the Rumin complex in $(M, H, g)$ and in the Heisenberg group.

The core of this section consists in the proof of an approximate homotopy formula

$$
I=d_{c} T_{M}+T_{M} d_{c}+S_{M},
$$

where the 'error term' $S_{M}$ has the maximal regularizing property compatible with the regularity of $M$, and $T_{M}$ enjoys the natural continuity properties between Sobolev spaces on $M$. The proof will be carried out in two steps: first (Lemma 6.1), we shall prove an approximate homotopy formula akin to (63), where $S_{M}$ 'gains only one horizontal derivative', and then iterating (63), we obtain the desired approximate homotopy formula, where $S_{M}$ has the maximal regularizing property compatible with the regularity of $M$.

As in Definition 4.12 , let now $\left\{\chi_{j}\right\}$ be a partition of the unity subordinate to the atlas $\mathcal{U}:=\left\{B\left(x_{j}, \rho\right), \phi_{x_{j}}\right\}$ of Lemma 4.11. From now on, for the sake of simplicity, we shall write $\phi_{j}:=\phi_{x_{j}}$. We stress again that $\phi_{j}^{-1}\left(\operatorname{supp} \chi_{j}\right) \subset B(e, 1)$.

If $u \in L^{p}\left(M, E_{0}^{\bullet}\right)$, we have

$$
u=\sum_{j} \chi_{j} u .
$$

We can write

$$
\chi_{j} u=\left(\phi_{j}^{-1}\right)^{\#} \phi_{j}^{\#}\left(\chi_{j} u\right)=:\left(\phi_{j}^{-1}\right)^{\#} v_{j} .
$$

We use now the homotopy formula in $\mathbb{H}^{n}$ (see Theorem 5.12):

$$
v_{j}=d_{c} T v_{j}+T d_{c} v_{j}+S v_{j} \quad \text { in } B(e, 1) .
$$

Without loss of generality, we can assume that $R>0$ in the definition of the kernel of $T$ has been chosen in such a way that the $R$-neighborhood of $\phi_{j}^{-1}\left(\operatorname{supp} \chi_{j}\right) \subset B(e, 1)$. In particular, $v_{j}-d_{c} T v_{j}-T d_{c} v_{j}$ is supported in $B(e, 1)$ and therefore also $S v_{j}$ is supported in $B(e, 1)$. 
In particular, $\left(\phi_{j}^{-1}\right)^{\#}\left(d_{c} T v_{j}+T d_{c} v_{j}+S v_{j}\right)$ is supported in $\phi_{j}(B(e, 1))$ so that it can be continued by zero on $M$.

Thus

$$
\begin{aligned}
u= & \sum_{j}\left(\phi_{j}^{-1}\right)^{\#}\left(d_{c} T v_{j}+T d_{c} v_{j}+S v_{j}\right) \\
= & d_{c} \sum_{j}\left(\phi_{j}^{-1}\right)^{\#} T \phi_{j}^{\#}\left(\chi_{j} u\right) \\
& +\sum_{j}\left(\left(\phi_{j}^{-1}\right)^{\#} T \phi_{j}^{\#} \chi_{j}\right) d_{c} u-\sum_{j}\left(\phi_{j}^{-1}\right)^{\#} T \phi_{j}^{\#}\left(\left[\chi_{j}, d_{c}\right] u\right) \\
& +\sum_{j}\left(\phi_{j}^{-1}\right)^{\#}\left(S \phi_{j}^{\#} \chi_{j}\right) u .
\end{aligned}
$$

We set

$$
\mathbf{T} u:=\sum_{j}\left(\phi_{j}^{-1}\right)^{\#} T \phi_{j}^{\#}\left(\chi_{j} u\right)
$$

and

$$
\mathbf{S} u:=\sum_{j}\left(\phi_{j}^{-1}\right)^{\#} S \phi_{j}^{\#}\left(\chi_{j} u\right)-\sum_{j}\left(\phi_{j}^{-1}\right)^{\#} T \phi_{j}^{\#}\left(\left[\chi_{j}, d_{c}\right] u\right) .
$$

Lemma 6.1. Let $(M, H, g)$ be a bounded $C^{k}$-geometry sub-Riemannian contact manifold with $k \geqslant 3$. If $2 \leqslant \ell \leqslant k-1$ and $\mathbf{T}$ and $\mathbf{S}$ are defined in (64) and (65), then the following homotopy formula holds:

$$
I=d_{c} \mathbf{T}+\mathbf{T} d_{c}+\mathbf{S}
$$

In particular, $\mathbf{S} d_{c}=d_{c} \mathbf{S}$. In addition, if $1 \leqslant h \leqslant 2 n+1$, the following maps are continuous:

(i) $\mathbf{T}: W^{-1, p}\left(M, E_{0}^{h+1}\right) \rightarrow L^{p}\left(M, E_{0}^{h}\right)$ if $h \neq n$, whereas $\mathbf{T}: W^{-2, p}\left(M, E_{0}^{n+1}\right) \rightarrow L^{p}$ $\left(M, E_{0}^{n}\right)$;

(ii) $\mathbf{T}: L^{p}\left(M, E_{0}^{h}\right) \rightarrow W^{1, p}\left(M, E_{0}^{h-1}\right)$ if $h \neq n+1$, whereas $\mathbf{T}: L^{p}\left(M, E_{0}^{n+1}\right) \rightarrow W^{2, p}$ $\left(M, E_{0}^{n}\right)$;

(iii) if $1 \leqslant \ell \leqslant k$, then $\mathbf{S}: W^{\ell-1, p}\left(M, E_{0}^{h}\right) \longrightarrow W^{\ell, p}\left(M, E_{0}^{h}\right)$.

Proof. First of all, we note that if $\alpha$ is supported in $\phi_{j}(B(e, \lambda))$, then by Definition 4.9 , the norms

$$
\|\alpha\|_{W^{m, p}\left(M, E_{0}^{\bullet}\right)} \quad \text { and } \quad\left\|\phi_{j}^{\#} \alpha\right\|_{W^{m, p}\left(\mathbb{H}^{n}, E_{0}^{\bullet}\right)}
$$

are equivalent for $-k \leqslant m \leqslant k$, with equivalence constants independent of $j$. Thus, assertions (i) and (ii) follow straightforwardly from Theorem 5.14.

To get (iii), we only need to note that the operators $\left(\phi_{j}^{-1}\right)^{\#} T \phi_{j}^{\#}\left[\chi_{j}, d_{c}\right]$ are bounded $W^{\ell-1, p}\left(M, E_{0}^{\bullet}\right) \rightarrow W^{\ell, p}\left(M, E_{0}^{\bullet}\right)$ in every degree. Indeed, by Proposition 2.14 , the differential operator $\phi_{j}^{\#}\left[\chi_{j}, d_{c}\right]\left(\phi_{j}^{-1}\right)^{\#}$ in $\mathbb{H}^{n}$ has order 1 if $h=n$ and order 0 if $h \neq n$. Since the kernel of $T$ can be estimated by a kernel of type 2 if $T$ acts on forms of 
degree $h=n$ and of type 1 if it acts on forms of degree $h \neq n$, the assertion follows straightforwardly.

Summing up in $j$ and taking into account that the sum is locally finite, we obtain

$$
\begin{aligned}
& \left\|\sum_{j}\left(\phi_{j}^{-1}\right)^{\#} T \phi_{j}^{\#}\left[\chi_{j}, d_{c}\right] u\right\|_{W^{\ell, p}\left(M, E_{0}^{\bullet}\right)} \leqslant \sum_{j}\left\|\left(\phi_{j}^{-1}\right)^{\#} T \phi_{j}^{\#}\left[\chi_{j}, d_{c}\right] u\right\|_{W^{\ell, p}\left(\phi_{j}(B(e, 1)), E_{0}^{\bullet}\right)} \\
& \quad \leqslant C \sum_{j}\left\|T \phi_{j}^{\#}\left[\chi_{j}, d_{c}\right] u\right\|_{W^{\ell, p}\left(B(e, 1), E_{0}^{\bullet}\right)} \leqslant C \sum_{j}\left\|\phi_{j}^{\#} u\right\|_{W^{\ell-1, p}\left(B(e, 1), E_{0}^{\bullet}\right)} \\
& \quad \leqslant C\|u\|_{W^{\ell-1, p}\left(M, E_{0}^{\bullet}\right)}
\end{aligned}
$$

Now the following global homotopy formula holds in $M$.

Theorem 6.2. Let $(M, H, g)$ be a bounded $C^{k}$-geometry sub-Riemannian contact manifold, $k \geqslant 3$. Then

$$
I=d_{c} T_{M}+T_{M} d_{c}+S_{M}
$$

where

$$
T_{M}:=\left(\sum_{i=0}^{k-1} \mathbf{S}^{i}\right) \mathbf{T}, \quad S_{M}:=\mathbf{S}^{k},
$$

and $\mathbf{T}$ and $\mathbf{S}$ are defined in (64) and (65).

Moreover,

$$
d_{c} S_{M} u=S_{M} d_{c} u,
$$

and if $1 \leqslant h \leqslant 2 n+1$, the following maps are continuous:

(i) $T_{M}: W^{-1, p}\left(M, E_{0}^{h+1}\right) \rightarrow L^{p}\left(M, E_{0}^{h}\right)$ if $h \neq n$, whereas $T_{M}: W^{-2, p}\left(M, E_{0}^{n+1}\right) \rightarrow$ $L^{p}\left(M, E_{0}^{n}\right)$

(ii) $T_{M}: L^{p}\left(M, E_{0}^{h}\right) \rightarrow W^{1, p}\left(M, E_{0}^{h-1}\right)$ if $h \neq n+1$, whereas $T_{M}: L^{p}\left(M, E_{0}^{n+1}\right) \rightarrow$ $W^{2, p}\left(M, E_{0}^{n}\right)$

(iii) $S_{M}: L^{p}\left(M, E_{0}^{h}\right) \rightarrow W^{k-1, p}\left(M, E_{0}^{h}\right)$.

Proof. By (68),

$$
\begin{aligned}
d_{c} T_{M}+T_{M} d_{c}+S_{M} \\
=d_{c}\left(\sum_{i=0}^{k-1} S^{i}\right) T+\left(\sum_{i=0}^{k-1} S^{i}\right) \tilde{T} d_{c}+S^{k} \\
=\sum_{i=0}^{k-1} S^{i}\left(d_{c} T+T d_{c}\right)+S^{k} \\
=\sum_{i=0}^{k-1} S^{i}(I-S)+S^{k}=I .
\end{aligned}
$$

Then statements (i)-(iii) follow straightforwardly from (i)-(iii) of Lemma 6.1. 


\section{Large scale geometry of contact sub-Riemannian manifolds}

Theorems 1.2 and 1.5 are the key to proving that the validity of global Poincaré inequalities is equivalent to the vanishing of $\ell^{q, p}$ cohomology, a large scale invariant of metric spaces. This equivalence will be established in [47]. By large scale invariant, we mean preserved, under uniform local assumptions, by quasi-isometries, i.e., maps $f$ between metric spaces, which satisfy

$$
-C+\frac{1}{L} d\left(x, x^{\prime}\right) \leqslant d\left(f(x), f\left(x^{\prime}\right)\right) \leqslant L d\left(x, x^{\prime}\right)+C,
$$

for suitable positive constants $L$ and $C$.

Avoiding the general metric definition of $\ell^{q, p}$ cohomology, let us give a construction valid for bounded geometry Riemannian manifolds with uniform vanishing of cohomology (the cohomology of an $R^{\prime}$-ball dies when restricted to a concentric $R$-ball, where the radius $R^{\prime}$ depends only on the radius $R$ ). First, one defines the $\ell^{q, p}$ cohomology of a simplicial complex: it is the quotient of the space of $\ell^{p}$ simplicial cocycles by the image of $\ell^{q}$ simplicial cochains by the coboundary operator. One shows that $\ell^{q, p}$ cohomology is a quasi-isometry invariant of simplicial complexes with bounded geometry (i.e., bounded number of simplices through a vertex) and uniform vanishing of cohomology. Then one observes that every bounded geometry Riemannian manifold is quasi-isometric to such a simplicial complex.

Under similar boundedness and uniformity assumptions, one can show [47] that various locally acyclic complexes can be used to compute $\ell^{q, p}$ cohomology. For contact sub-Riemannian manifolds, one can use either the exterior differential or Rumin's differential. As alluded to above, the building blocks are interior estimates and global smoothing, i.e., Theorems 1.2 and 1.5 and their Riemannian analogues. It follows that a global Poincaré inequality holds if and only if a global $\mathbb{H}$-Poincaré inequality holds.

Using the Riemannian Hodge Laplacian, Müller et al. prove a Poincaré inequality Poincaré $2, q$ for the exterior differential on the Riemannian Heisenberg group [44, Lemma 11.2], under the assumption $\frac{1}{2}-\frac{1}{q}=\frac{1}{2 n+1}$. Therefore, their result combined with [47] provides an alternative proof of part of Corollary 1.4. We note that in degree $h=n+1$, they miss the sharp exponent, given by our condition $E(n+1,2, q, n)$.

The advantage of Rumin's Laplacian over its Riemannian sibling is its scale invariance. This allows us to apply the theory of singular integral operators to treat $\ell^{q, p}$ cohomology for all $p$ and to get the sharp exponent in degree $h=n+1$. The drawback of the Rumin complex is that interior Poincaré inequalities become hard.

\subsection{Three-dimensional Lie groups}

There are four 3-dimensional Lie algebras that cannot be generated by a pair of vectors: the abelian Lie algebra $\mathbb{R}^{3}, \mathfrak{d} \mathfrak{i l}(2)$, the direct sum $\mathfrak{d i l}(1) \oplus \mathbb{R}$, where $\operatorname{dil}(n)$ denotes the Lie algebra of the group of dilations and translations of $\mathbb{R}^{n}$, and the solvable unimodular Lie algebra $\mathfrak{s o l}$. The Lie groups corresponding to other 3-dimensional Lie algebras admit left-invariant contact structures. All left-invariant sub-Riemannian metrics have bounded geometry, so Theorem 1.5 applies. When simply connected, they satisfy all uniform local 
assumptions required for the identification of the $\mathbb{H}$-Poincaré ${ }_{p, q}$ inequality with vanishing of $\ell^{q, p}$ cohomology and its quasi-isometry invariance. Here are examples.

The Heisenberg group $\mathbb{H}^{1}$ is covered by Theorem 1.1. Note that the corresponding facts about $\ell^{q, p}$ cohomology are new.

$\widetilde{M}_{1}:=\widetilde{\operatorname{Mot}\left(E^{2}\right)}$, the universal covering of the group of planar Euclidean motions, is quasi-isometric to Euclidean 3 -space $E^{3}$. Its $\ell^{q, p}$ cohomology vanishes if and only if $\frac{1}{p}-\frac{1}{q} \geqslant \frac{1}{3}$ (this is the Euclidean analogue of Theorem 1.1). Therefore, assuming $1<p \leqslant$ $q<\infty$, the $\mathbb{H}$-Poincaré $p, q$ inequality holds for this group if and only if $\frac{1}{p}-\frac{1}{q} \geqslant \frac{1}{3}$ in all degrees.

$\widetilde{M}_{2}:=\widetilde{\operatorname{SL}(2, \mathbb{R})}$, the universal covering of $\operatorname{SL}(2, \mathbb{R})$, is quasi-isometric to $\operatorname{PSL}(2, \mathbb{R}) \times \mathbb{R}$. In degree 1 , its $\ell^{p, p}$-cohomology vanishes for all $p>1$; see [46]. Since PSL(2, $\left.\mathbb{R}\right)$ acts isometrically and simply transitively on hyperbolic plane $H^{2}$, it is quasi-isometric to $H^{2}$. Since the $\ell^{p, p}$-cohomology of $H^{2}$ in degree 1 is Hausdorff and non-zero, the Künneth formula of [29] applies, and the $\ell^{p, p}$-cohomology in degree 2 of the product does not vanish because the $\ell^{p, p}$-cohomology in degree 1 of the line does not vanish. We conclude that, assuming $1<p<\infty$, the $\mathbb{H}$-Poincaré -P, $_{p}$ inequality holds in degree 1 and only in degree 1 .

\subsection{Other examples}

Next, we describe a few non-simply-connected examples. Then the quasi-isometry invariance holds only in degree 1 .

Let $M_{0}$ be the quotient of the Heisenberg group $\mathbb{H}^{1}$ by the discrete subgroup $\Gamma$ generated by two elements, one of which belongs to the center of $\mathbb{H}^{1}$. Let us equip it with the quotient contact structure and sub-Riemannian metric. $\Gamma$ is contained in a connected subgroup $L$ of $\mathbb{H}^{1}$ isomorphic to $\mathbb{R}^{2}$. This gives rise to a fibration $M_{0} \rightarrow L \backslash \mathbb{H}^{1}$, which is a line. The fibers of this map are tori with uniformly bounded diameters; therefore it is a quasi-isometry. The $\ell^{q, p}$ cohomology of the line is well understood; it vanishes only when $(q, p)=(\infty, 1)$. Therefore, assuming $1<p \leqslant q<\infty$, the $\mathbb{H}$-Poincaré ${ }_{p, q}$ inequality never holds for $M_{0}$ in degree 1 .

Let $M_{1}$ denote the unit cotangent bundle of the Euclidean plane $E^{2}$. It carries a tautological contact structure. The group $G_{1}$ of motions of the Euclidean plane, which is a semi-direct product of $\mathbb{R}^{2}$ with $S O(2)$, acts simply transitively on $M$, preserving the contact structure. Pick a $G_{1}$-invariant sub-Riemannian metric on $M$. By invariance, the bounded geometry assumption is satisfied. The projection $M \rightarrow E^{2}$ has uniformly bounded fibers; it is a quasi-isometry. Therefore $M$ and $E^{2}$ have isomorphic exact $\ell^{q, p}$ cohomologies in degree 1 . The $\ell^{q, p}$ cohomology of $E^{2}$ is well understood. It vanishes if and only if $\frac{1}{p}-\frac{1}{q} \geqslant \frac{1}{2}$. We conclude that, assuming $1<p \leqslant q<\infty$, the $\mathbb{H}$-Poincaré ${ }_{p, q}$ inequality holds for $M_{1}$ in degree 1 if and only if $\frac{1}{p}-\frac{1}{q} \geqslant \frac{1}{2}$.

Let us replace the Euclidean plane with the hyperbolic plane $H^{2}$. The construction is identical up to the structure of the identity component $G_{2}$ of the isometry group of the hyperbolic plane: it is isomorphic to $\operatorname{PSL}(2, \mathbb{R})$. The obtained sub-Riemannian manifold $M_{2}$ is quasi-isometric to $H^{2}$. The $\ell^{q, p}$ cohomology of $H^{2}$ in degree 1 is well understood. 
It vanishes only for $p=1$. We conclude that the $\mathbb{H}$-Poincaré -P $_{q}$ inequality never holds in degree 1 for $M_{2}$ if $1<p \leqslant q<\infty$.

\subsection{Further remarks}

In each degree $k$, for every $p$, there is an exponent $q=q(n, k)$ such that the $L^{q}$-norm of Rumin $k$-forms is a conformal invariant $\left(q(n, k)=\frac{2 n+2}{k}\right.$ if $k \leqslant n, q(n, k)=\frac{2 n+2}{k+1}$ if $k \geqslant$ $n+1)$. Therefore, in degree $k, \ell^{q(n, k-1), q(n, k)}$ cohomology of $2 n+1$-dimensional contact sub-Riemannian manifolds is a quasi-conformal invariant, and so is does the validity of an $\mathbb{H}$-Poincaré $q(n, k), q(n, k-1)$ inequality. We note that if $k<2 n+1$, for the Heisenberg group $\mathbb{H}^{n}$, these cohomology groups vanish, whereas they need not vanish for other examples. For instance, if $n=1, q(n, 1)=4$ and $q(n, 2)=2$, the $\ell^{4,2}$-cohomology in degree 2 of $\widetilde{M}_{1}$ does not vanish. This shows that $\widetilde{M}_{1}$ is not quasi-conformally equivalent to $\mathbb{H}^{1}$.

We see that Theorems 1.2 and 1.5 constitute useful tools for the geometric study of mappings between contact sub-Riemannian manifolds. Here are a few references about this emerging subject: [35] shows that two ways to take a quotient of a Heisenberg group by an isometry give rise to contact sub-Riemannian manifolds, which are not quasi-conformal. Moreover, [31] establishes the basic properties of quasi-regular maps, a study that has been continued in $[12,19,37]$.

Acknowledgments. The authors are happy to thank the referees for their comments and suggestions that improved the readability of the paper.

A.B. and B.F. are supported by the University of Bologna, funds for selected research topics, and by MAnET Marie Curie Initial Training Network, GNAMPA of INdAM (Istituto Nazionale di Alta Matematica 'F. Severi'), Italy, and PRIN of the MIUR, Italy.

P.P. is supported by MAnET Marie Curie Initial Training Network and Agence Nationale de la Recherche, ANR-10-BLAN 116-01 GGAA and ANR-15-CE40-0018 SRGI. P.P. gratefully acknowledges the hospitality of Isaac Newton Institute, EPSRC under grant EP/K032208/1, and Simons Foundation.

\section{References}

1. A. Baldi, M. Barnabei And B. Franchi, A recursive basis for primitive forms in symplectic spaces and applications to Heisenberg groups, Acta Math. Sin. (Engl. Ser.) 32(3) (2016), 265-285. MR 3456421.

2. A. BAldi And B. Franchi, Sharp a priori estimates for div-curl systems in Heisenberg groups, J. Funct. Anal. 265(10) (2013), 2388-2419. MR 3091819.

3. A. Baldi, B. Franchi And P. PAnsu, Gagliardo-Nirenberg inequalities for differential forms in Heisenberg groups, Math. Ann. 365(3-4) (2016), 1633-1667. MR 3521101.

4. A. BAldi, B. Franchi AND P. PANsu, $L^{1}$-Poincaré and Sobolev inequalities for differential forms in Euclidean spaces, Sci. China Math. 62(6) (2019), 1029-1040. MR 3951879.

5. A. Baldi, B. Franchi and P. Pansu, Duality and $L^{\infty}$ differential forms on Heisenberg groups, in preparation, 2020. 
6. A. Baldi, B. Franchi and P. Pansu, $L^{1}$-Poincaré inequalities for differential forms on Euclidean spaces and Heisenberg groups, Adv. Math. 366 (2020), 107084. MR 4070308.

7. A. Baldi, B. Franchi and P. Pansu, Orlicz spaces and endpoint Sobolev-Poincaré inequalities for differential forms in Heisenberg groups, Matematiche (Catania) 75 (2020), $167-194$.

8. A. Baldi, B. Franchi, N. Tchou and M. C. Tesi, Compensated compactness for differential forms in Carnot groups and applications, Adv. Math. 223(5) (2010), 1555-1607.

9. A. Baldi, B. Franchi And M. C. Tesi, Compensated compactness in the contact complex of Heisenberg groups, Indiana Univ. Math. J. 57 (2008), 133-186.

10. A. Baldi, B. Franchi And M. C. Tesi, Hypoellipticity, fundamental solution and Liouville type theorem for matrix-valued differential operators in Carnot groups, J. Eur. Math. Soc. (JEMS) 11(4) (2009), 777-798.

11. A. Baldi, B. Franchi and F. Tripaldi, Gagliardo-Nirenberg inequalities for horizontal vector fields in the Engel group and in the seven-dimensional quaternionic Heisenberg group, in Geometric Methods in PDE's, Springer INdAM Series, Volume 13, pp. 287-312 (Springer, Cham, 2015). MR 3617226.

12. Z. M. Balogh, K. Fässler And K. Peltonen, Uniformly quasiregular maps on the compactified Heisenberg group, J. Geom. Anal. 22(3) (2012), 633-665. MR 2927672.

13. A. Bernig, Natural operations on differential forms on contact manifolds, Differential Geom. Appl. 50 (2017), 34-51. MR 3588639.

14. A. Bonfiglioli, E. LAnconelli And F. Uguzzoni, Stratified Lie groups and Potential Theory for Their Sub-Laplacians, Springer Monographs in Mathematics, (Springer, Berlin, 2007). MR 2363343.

15. J. Bourgain AND HaÏm Brezis, New estimates for elliptic equations and Hodge type systems, J. Eur. Math. Soc. (JEMS) 9(2) (2007), 277-315. MR 2293957 (2009h:35062).

16. R. Bryant, M. Eastwood, A. Rod Gover and K. Neusser, Some differential complexes within and beyond parabolic geometry, Preprint, 2011, arXiv:1112.2142.

17. L. Capogna, D. Danielli and N. Garofalo, Subelliptic mollifiers and a basic pointwise estimate of Poincaré type, Math. Z. 226(1) (1997), 147-154. MR 1472145.

18. S. Chanillo and J. Van Schaftingen, Subelliptic Bourgain-Brezis estimates on groups, Math. Res. Lett. 16(3) (2009), 487-501. MR 2511628 (2010f:35042).

19. K. Fässler, A. Lukyanenko AND K. Peltonen, Quasiregular mappings on sub-Riemannian manifolds, J. Geom. Anal. 26(3) (2016), 1754-1794. MR 3511457.

20. G. B. Folland, Subelliptic estimates and function spaces on nilpotent Lie groups, Ark. Mat. 13(2) (1975), 161-207. MR 0494315 (58 \#13215).

21. G. B. Folland And E. M. Stein, Hardy Spaces on Homogeneous Groups, Mathematical Notes, Volume 28, (Princeton University Press, Princeton, NJ, 1982). MR 657581 (84h:43027).

22. B. Franchi, C. E. Gutiérrez and R. L. Wheeden, Weighted Sobolev-Poincaré inequalities for Grushin type operators, Comm. Partial Differential Equations 19(3-4) (1994), 523-604. MR 1265808.

23. B. FrAnChi, G. Lu AND R. L. WheEden, Representation formulas and weighted Poincaré inequalities for Hörmander vector fields, Ann. Inst. Fourier (Grenoble) 45(2) (1995), 577-604. MR 1343563 (96i:46037).

24. B. Franchi, G. Lu and R. L. Wheeden, A relationship between Poincaré-type inequalities and representation formulas in spaces of homogeneous type, Int. Math. Res. Not. IMRN (1) (1996), 1-14. MR 1383947 (97k:26012).

25. B. Franchi, R. Serapioni and F. S. Cassano, Meyers-Serrin type theorems and relaxation of variational integrals depending on vector fields, Houston J. Math. 22(4) (1996), 859-890. MR 1437714. 
26. B. Franchi and R. P. Serapioni, Intrinsic Lipschitz graphs within Carnot groups, J. Geom. Anal. 26(3) (2016), 1946-1994. MR 3511465.

27. B. Franchi, R. Serapioni and F. S. Cassano, Regular submanifolds, graphs and area formula in Heisenberg groups, Adv. Math. 211(1) (2007), 152-203. MR 2313532 (2008h:49030).

28. S. Gallot, D. Hulin and J. Lafontaine, Riemannian Geometry, third edition, Universitext, (Springer, Berlin, 2004). MR 2088027.

29. V. M. Gol'dshtein, V. I. Kuz'minov And I. A. Shvedov, $L_{p}$-cohomology of warped cylinders, Sibirsk. Mat. Zh. 31(6) (1990), 55-63. MR 1097955.

30. M. Gromov, Carnot-Carathéodory spaces seen from within, in Sub-Riemannian Geometry, Progress in Mathematics, Volume 144, pp. 79-323 (Birkhäuser, Basel, 1996). MR 1421823 (2000f:53034).

31. J. Heinonen And I. Holopainen, Quasiregular maps on Carnot groups, J. Geom. Anal. 7(1) (1997), 109-148. MR 1630785.

32. B. HelfFer AND J. NouRRIGAT, Hypoellipticité maximale pour des opérateurs polynômes de champs de vecteurs, Progress in Mathematics, Volume 58, (Birkhäuser Boston Inc., Boston, MA, 1985). MR 897103 (88i:35029).

33. T. IwANiec And A. Lutoborski, Integral estimates for null Lagrangians, Arch. Ration. Mech. Anal. 125(1) (1993), 25-79. MR 1241286 (95c:58054).

34. D. JERISON, The Poincaré inequality for vector fields satisfying Hörmander's condition, Duke Math. J. 53(2) (1986), 503-523. MR 850547 (87i:35027).

35. Y. KIM, Quasiconformal conjugacy classes of parabolic isometries of complex hyperbolic space, Pacific J. Math. 270(1) (2014), 129-149. MR 3245851.

36. L. Lanzani And E. M. Stein, A note on div curl inequalities, Math. Res. Lett. 12(1) (2005), 57-61. MR 2122730 (2005m:58001).

37. A. Lukyanenko, Geometric mapping theory of the Heisenberg group, sub-Riemannian manifolds, and hyperbolic spaces, ProQuest LLC, Ann Arbor, MI, 2014, Ph.D. Thesis, University of Illinois at Urbana-Champaign. MR 3322035.

38. P. Maheux and L. SAloff-Coste, Analyse sur les boules d'un opérateur sous-elliptique, Math. Ann. 303(4) (1995), 713-740. MR 1359957 (96m:35049).

39. J. Martinet, Formes de contact sur les variétés de dimension 3, Lecture Notes in Mathematics, Volume 209, pp. 142-163 (Springer, Berlin, 1971). MR 0350771.

40. P. MattilA, Geometry of Sets and Measures in Euclidean Spaces: Fractals and Rectifiability, Cambridge Studies in Advanced Mathematics, Volume 44, (Cambridge University Press, Cambridge, 1995). MR 1333890.

41. D. MCDuff AND D. SAlamon, Introduction to Symplectic Topology, second edition, Oxford Mathematical Monographs, (The Clarendon Press, Oxford University Press, New York, 1998). MR 1698616.

42. D. Mitrea, M. Mitrea And S. Monniaux, The Poisson problem for the exterior derivative operator with Dirichlet boundary condition in nonsmooth domains, Commun. Pure Appl. Anal. 7(6) (2008), 1295-1333. MR 2425010.

43. R. MONTGOMery, A tour of Subriemannian Geometries, their Geodesics and Applications, Mathematical Surveys and Monographs, Volume 91, (American Mathematical Society, Providence, RI, 2002). MR 1867362 (2002m:53045).

44. D. Müller, M. M. Peloso And F. Ricci, Analysis of the Hodge Laplacian on the Heisenberg group, Mem. Amer. Math. Soc. 233(1095) (2015), vi+91. MR 3289035.

45. P. PAnsu, Métriques de Carnot-Carathéodory et quasiisométries des espaces symétriques de rang un, Ann. of Math. (2) 129(1) (1989), 1-60. MR 979599 (90e:53058).

46. P. Pansu, Cohomologie $L^{p}$ en degré 1 des espaces homogènes, Potential Anal. 27(2) (2007), 151-165. MR 2322503. 
47. P. PAnsu, Cup-products in $l^{q, p}$-cohomology: discretization and quasi-isometry invariance, Preprint, 2017, arXiv:1702.04984.

48. P. Pansu and M. Rumin, On the $\ell^{q, p}$ cohomology of Carnot groups, Ann. H. Lebesgue 1 (2018), 267-295. MR 3963292.

49. P. Pansu And F. TRIPAldi, Averages and the $\ell^{q, 1}$ cohomology of Heisenberg groups, Ann. Math. Blaise Pascal 26(1) (2019), 81-100. (en).

50. M. Rumin, Formes différentielles sur les variétés de contact, J. Differential Geom. 39(2) (1994), 281-330. MR 1267892 (95g:58221).

51. M. Rumin, Differential geometry on C-C spaces and application to the Novikov-Shubin numbers of nilpotent Lie groups, C. R. Acad. Sci. Paris Sér. I Math. 329(11) (1999), 985-990. MR 1733906 (2001g:53063).

52. M. Rumin, Sub-Riemannian limit of the differential form spectrum of contact manifolds, Geom. Funct. Anal. 10(2) (2000), 407-452. MR 1771424 (2002f:53044).

53. M. Rumin, Around heat decay on forms and relations of nilpotent Lie groups, Séminaire de Théorie Spectrale et Géométrie, Volume 19, pp. 123-164. MR 1909080 (2003f:58062).

54. M. Rumin, An introduction to spectral and differential geometry in Carnot-Carathéodory spaces, Rend. Circ. Mat. Palermo (2) Suppl. 75 (2005), 139-196. MR 2152359 (2006g:58053).

55. G. Schwarz, Hodge Decomposition-A Method for Solving Boundary Value Problems, Lecture Notes in Mathematics, Volume 1607, (Springer, Berlin, 1995). MR 1367287 (96k:58222).

56. E. M. Stein, Harmonic Analysis: Real-variable Methods, Orthogonality, and Oscillatory Integrals, Princeton Mathematical Series, Volume 43, (Princeton University Press, Princeton, NJ, 1993). With the assistance of Timothy S. Murphy, Monographs in Harmonic Analysis, III. MR 1232192 (95c:42002).

57. N. Th. Varopoulos, L. Saloff-Coste and T. Coulhon, Analysis and Geometry on Groups, Cambridge Tracts in Mathematics, Volume 100, (Cambridge University Press, Cambridge, 1992). MR 1218884 (95f:43008).

58. F. W. WARNER, Foundations of Differentiable Manifolds and Lie Groups, Graduate Texts in Mathematics, Volume 94, (Springer, New York, 1983). Corrected reprint of the 1971 edition. MR 722297. 